US Army Corps

of Engineers ${ }_{\circledast}$

Engineer Research and

Development Center

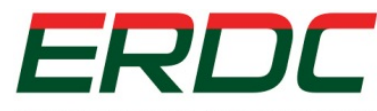

INNOVATIVE SOLUTIONS for a safer, better world

Military Facilities Engineering Technology

\title{
Facility Energy Performance Benchmarking in a Data-Scarce Environment
}

Sanat S. Bhole, Julie L. Webster, Matt D. Hiett

August 2017

and Nicholas M. Josefik

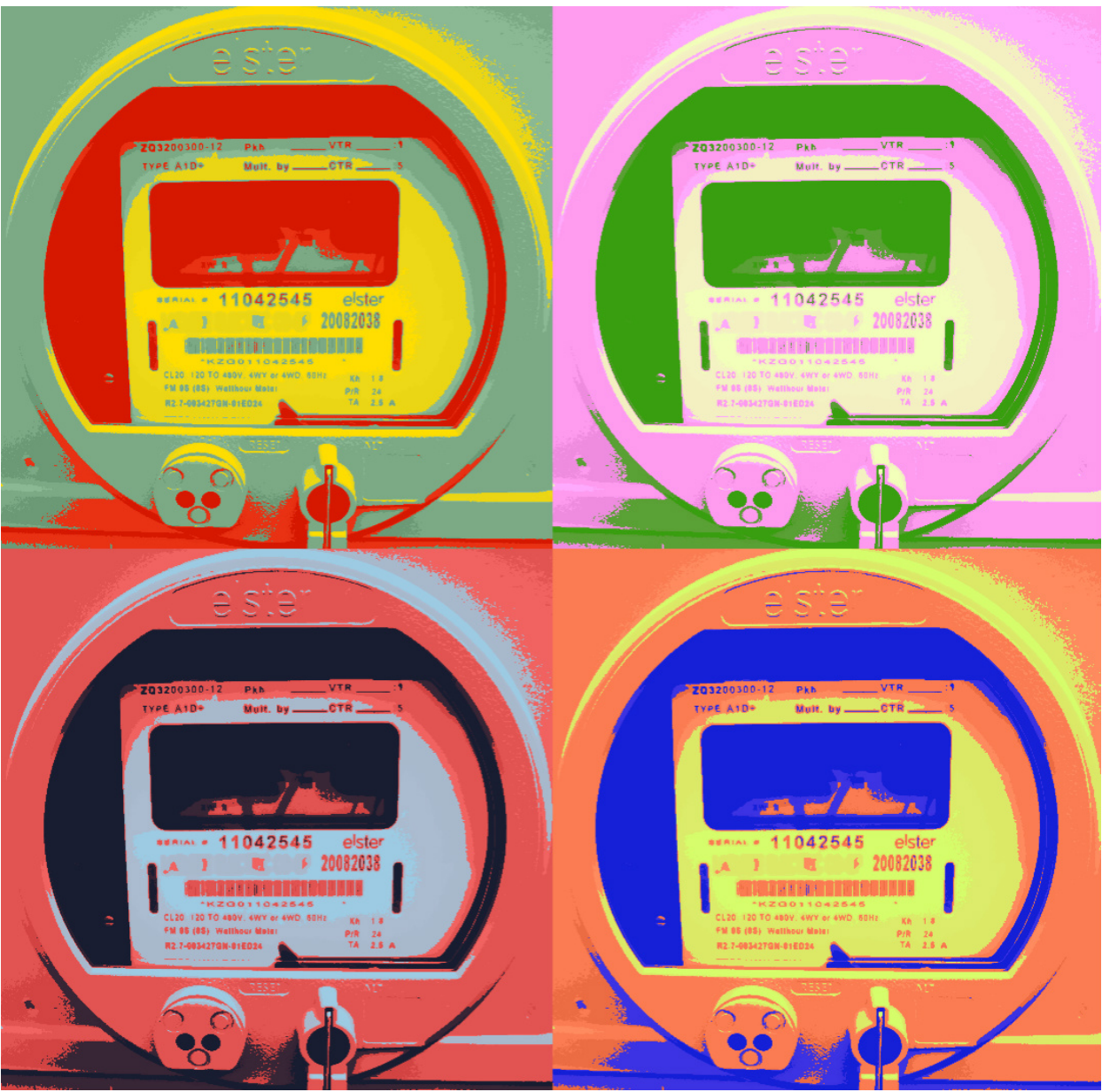

Approved for public release; distribution is unlimited. 
The U.S. Army Engineer Research and Development Center (ERDC) solves the nation's toughest engineering and environmental challenges. ERDC develops innovative solutions in civil and military engineering, geospatial sciences, water resources, and environmental sciences for the Army, the Department of Defense, civilian agencies, and our nation's public good. Find out more at www.erdc.usace.army.mil.

To search for other technical reports published by ERDC, visit the ERDC online library at http:// acwc.sdp.sirsi.net/ client/ default. 


\section{Facility Energy Performance Benchmarking in a Data-Scarce Environment}

Sanat S. Bhole, Julie L. Webster, Matt D. Hiett, and Nicholas M. Josefik

Construction Engineering Research Laboratory

U.S. Army Engineer Research and Development Center

2902 Newmark Drive

Champaign, IL 61822

Final report

Approved for public release; distribution is unlimited.

Prepared for U.S. Army Corps of Engineers

Washington, DC 20314-1000

Under Project P2 370256 "High Performance Building Operations - Decision Analysis for Energy (FY12-15)" 


\section{Abstract}

Current federal, Department of Defense (DoD), and Army energy-efficiency goals require a proactive approach to investment, building operations, and energy savings. Much responsibility for meeting these requirements is assigned to Army installation staff, who often have difficulties identifying and interpreting the applicable mandates. To address this problem, the research group began work to develop an intelligent framework that describes and clarifies interrelationships among energy efficiency, component maintenance and renewal, and mission requirements to support an integrated investment strategy that minimizes total cost of ownership (TCO). The main thrusts of the study were to develop integrated investment decision models, identify DoD facility Energy Use Intensity (EUI) benchmarks in a data-scarce environment, and analyze occupant-, system-, and component-level faults contributing to energy inefficiency.

A methodology for developing DoD-specific facility EUIs will serve as a decision framework for actions involving buildings with the highest EUIs. Thus, Army-specific benchmarking results will support more cost-effective component-renewal investment strategies. Altering the timing and grouping of investments can improve the energy efficiency to lower the TCO throughout the facility life cycle. This research will help the Army more effectively implement energy improvements to meet and exceed energy-efficiency requirements.

DISCLAIMER: The contents of this report are not to be used for advertising, publication, or promotional purposes. Citation of trade names does not constitute an official endorsement or approval of the use of such commercial products. All product names and trademarks cited are the property of their respective owners. The findings of this report are not to be construed as an official Department of the Army position unless so designated by other authorized documents. 


\section{Contents}

Abstract............................................................................................................................. ii

Figures and Tables..............................................................................................................v

Preface .........................................................................................................................vii

Unit Conversion Factors..................................................................................................vifi

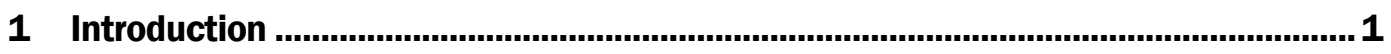

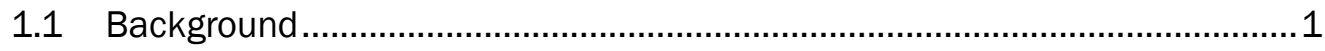

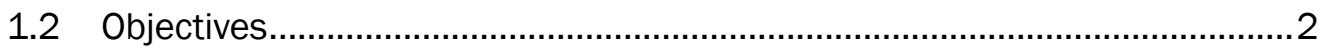

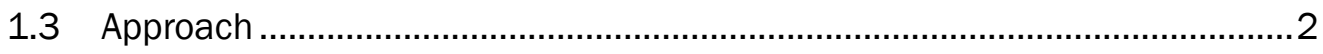

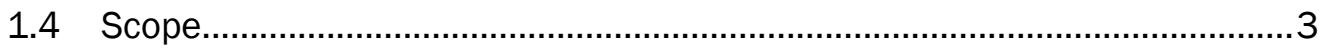

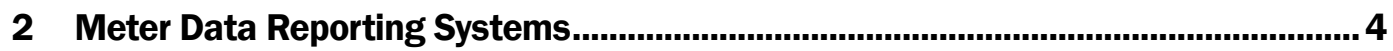

2.1 Installation-level metering.................................................................... 4

2.1.1 Defense Utility Energy Reporting System (DUERS) ............................................ 4

2.1.2 Army Energy and Water Reporting System (AEWRS) .......................................... 5

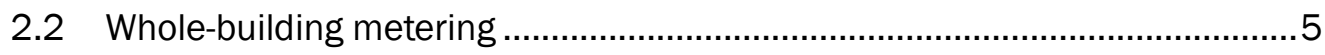

2.2.1 Meter Data Management System (MDMS) ....................................................... 5

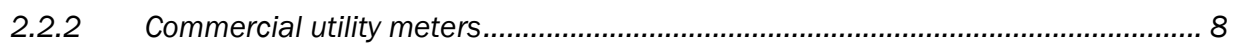

3 Building Selections .....................................................................................................10

3.1 Priority Army building types..............................................................10

3.2 Building attributes ...........................................................................11

3.2.1 EISA-required building attributes ..................................................................... 11

3.2.2 Building attributes in Army's HQIIS .................................................................. 12

3.2.3 DoD data analytics and integration support requirements ................................. 13

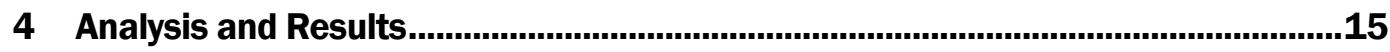

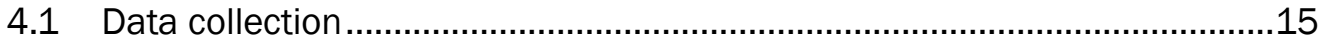

4.2 Insufficient data.................................................................................. 17

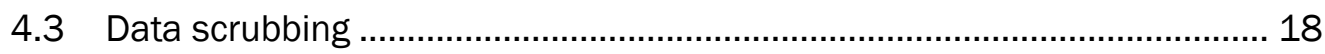

4.4 Data selection ............................................................................... 20

4.4.1 Estimating missing data-Microsoft Excel LINEST function.................................... 21

4.4.2 Estimating missing data-proportional method ................................................ 23

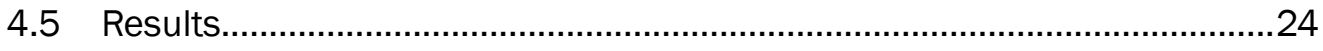

4.5.1 Parameter relationships: construction year and construction type.......................25

4.5.2 Parameter relationships: floor area .................................................................. 29

4.5.3 Parameter relationships: location ...................................................................... 33

4.5.4 Parameter relationships between occupancy and usage ................................... 38

4.6 Establishing benchmarks.......................................................... 39

4.7 Comparison of Army data and commercial studies.................................. 43

4.7.1 Commercial Buildings Energy Consumption Survey (CBECS)............................... 44 


\begin{tabular}{|c|c|}
\hline 7.2 & 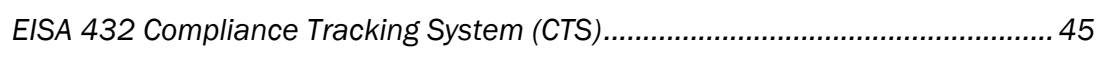 \\
\hline 7.3 & .45 \\
\hline 4.7.4 & New York City \\
\hline 4.7 .5 & 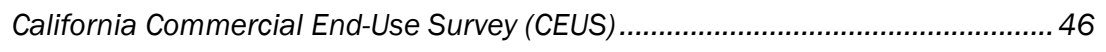 \\
\hline 4.7.6 & Nonresidential Building Energy Use Disclosure Program (CEC).... \\
\hline 4.7 .7 & f comparisons. \\
\hline
\end{tabular}

5 Conclusions and Recommendations ..............................................................48

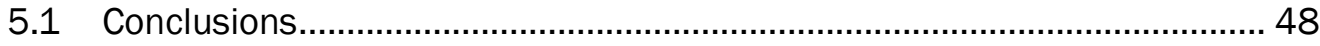

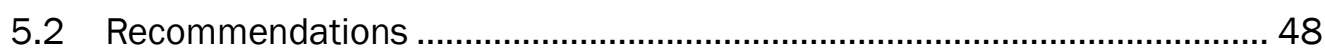

5.2.1 Improve data in Army real property databases ................................................... 48

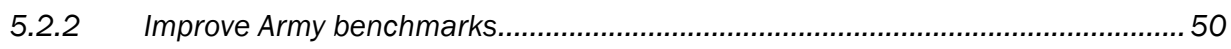

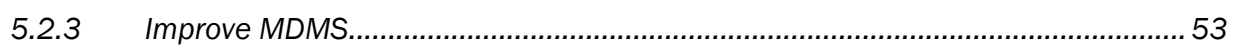

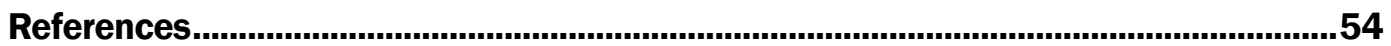

Appendix: Building Attributes Used in Data Analyses ...................................................56

Abbreviations and Acronyms................................................................................58

Report Documentation Page 


\section{Figures and Tables}

\section{Figures}

Figure 1. International Energy Conservation Code (IECC) climate zone map.....................12

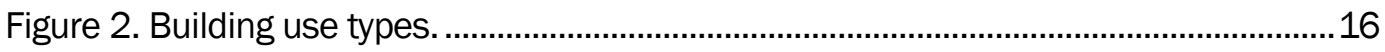

Figure 3. Building climate zone locations........................................................................16

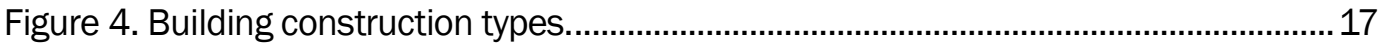

Figure 5. The ‘datacleanup' tab of the Excel tool............................................................19

Figure 6. Building 1 actual data also highlighting one estimated month..........................22

Figure 7. Building 1 actual data also highlighting three estimated months........................23

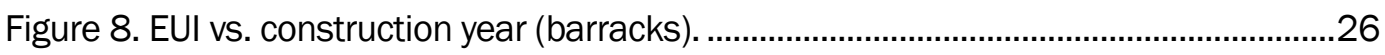

Figure 9. EUI vs. construction year (DFACs). ................................................................ 27

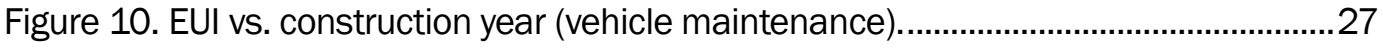

Figure 11. Construction year vs. construction type...........................................................28

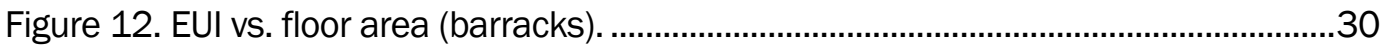

Figure 13. Annual energy consumption vs. floor area (barracks).......................................30

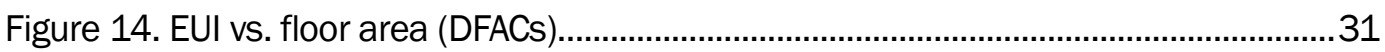

Figure 15. Annual energy consumption vs. floor area (DFACs). ............................................31

Figure 16. EUI vs. floor area (vehicle maintenance). ......................................................32

Figure 17. Annual energy consumption vs. floor area (vehicle maintenance)....................32

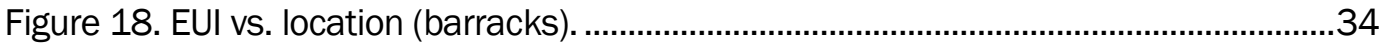

Figure 19. Electric and gas EUI vs. location (barracks). ..................................................34

Figure 20. Electric and gas annual energy consumption vs. location (barracks)...............35

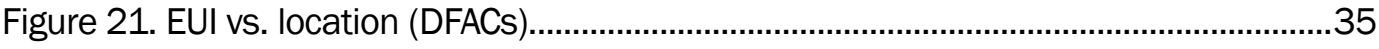

Figure 22. Electric and gas EUI vs. location (DFACs). .......................................................36

Figure 23. Electric and gas annual energy consumption vs. location (DFACs)..................36

Figure 24. EUI vs. location (vehicle maintenance). ............................................................37

Figure 25. Electric and gas EUI vs. location (vehicle maintenance)....................................37

Figure 26. Electric and gas annual energy consumption vs. location (vehicle

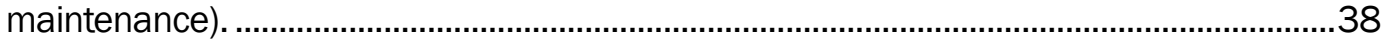

Figure 27. Barracks benchmark quartiles. ........................................................................... 41

Figure 28. DFAC benchmark quartiles........................................................................

Figure 29. Vehicle maintenance benchmark quartiles. ..........................................................43

\section{Tables}

Table 1. MDMS metering program status as of 18 September 2015. ................................ 7

Table 2. Sample MDMS export. .................................................................................... 8

Table 3. Example report generated by a private utility provider. ........................................... 9 
Table 4. 3Q FY15 Barracks statistics in the Army real property database. ........................10

Table 5. 3QFY15 Vehicle maintenance shop statistics in the Army real property database.

Table 6. 3QFY15 Dining facility statistics in the Army Real Property database. .................11

Table 7. Energy-relevant building attributes in HQIIS. .........................................................13

Table 8. EPA Portfolio Manager building attributes by facility type (EEIM)...........................14

Table 9. Functions in Excel data scrubbing tool. ...............................................................19

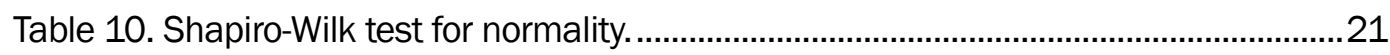

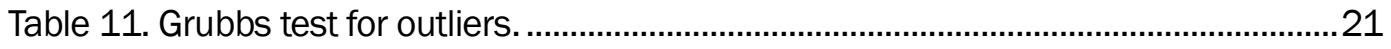

Table 12. Actual vs. estimated energy usage (1 month, LINSET function, Building

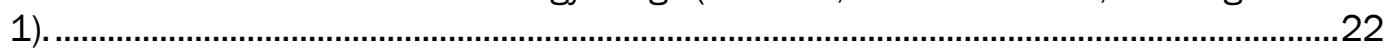

Table 13. Actual vs. estimated energy usage (3 months, LINSET function, Building

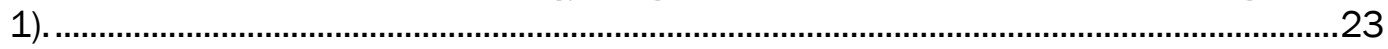

Table 14. Actual vs. estimated energy usage (3 months, proportional method,

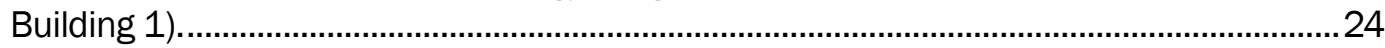

Table 15. Actual vs. estimated energy usage (3 months, proportional method,

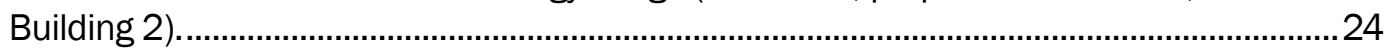

Table 16. Construction year and construction type mean EUls. .........................................28

Table 17. Construction year and construction type median EUls......................................29

Table 18. Data spread/variation (barracks).......................................................................39

Table 19. Data spread/variation (DFACs)...........................................................................39

Table 20. Data spread/variation (vehicle maintenance). ...................................................39

Table 21. Barracks quartile values.................................................................................... 41

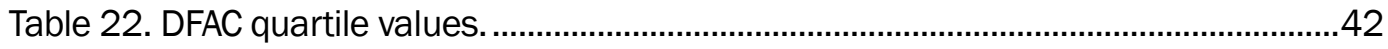

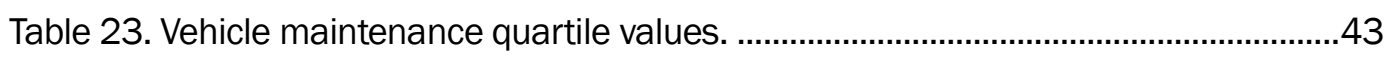

Table 24. Commercial studies building EUls...................................................................44

Table 25. Commercial study building datasets. .................................................................4

Table 26. Existing building attributes in HQIIS that are often not populated with

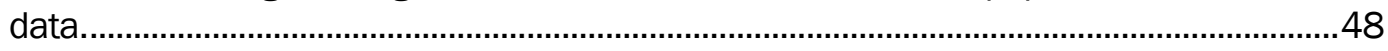

Table 27. Building attributes for Army to consider tracking in the future (EIA)...................49

Table 28. Useful attributes tracked in the IFS database..................................................49

Table 29. Building priority for future benchmarking studies. .............................................51 


\section{Preface}

This study was conducted for Headquarters, U.S. Army Corps of Engineers under Program Element 622784T41, "Military Facilities Engineering Technology"; Project P2 370256, "High Performance Building Operations- Decision Analysis for Energy (FY12- 15)." The technical monitor was Kurt Kinnevan, CEERD-CZT.

The work was performed by the Energy Branch of the Facilities Division (CEERD-CFM), U.S. Army Engineer Research and Development Center, Construction Engineering Research Laboratory (ERDC-CERL). At the time of publication, Dr. AndrewJ . Nelson was Chief, CEERD-CFE; Donald K. Hicks was Chief, CEERD-CF; and Kurt Kinnevan was the Technical Director for Adaptive and Resilient Installations. The Deputy Director of ERDCCERL was Dr. Kirankumar Topudurti and the Director was Dr. Ilker Adiguzel.

The Commander of ERDC was COL Bryan S. Green and the Director was Dr. David W. Pittman. 


\section{Unit Conversion Factors}

\begin{tabular}{|l|l|l|}
\hline Multiply & By & To Obtain \\
\hline kWh & 0.001 & Watt-hour \\
\hline MWh & 0.001 & kWh \\
\hline kBTUh & 0.001 & BTUh \\
\hline BTUh & 3412.142 & kWh \\
\hline kBTUh & 3.412142 & kWh \\
\hline BTUh & 1028 (varies) & cfh \\
\hline kW & 29.3 & Therm \\
\hline cf & 96.7 (often rounded to100) & Therm \\
\hline BTU & 100,000 & Therm \\
\hline kBTU & 100 & Therm \\
\hline BTU & 1 mil & MBTU [or MmBTU if using Roman Numerals] \\
\hline Days & 28.2422 & February \\
\hline
\end{tabular}




\section{Introduction}

\subsection{Background}

Current federal government, Department of Defense (DoD), and Army energy-utilization mandates require a proactive approach to military infrastructure investment, building operations, and energy efficiency. Implementing these mandates and achieving DoD objectives is a responsibility shared at many levels, including Army facility management personnel. Meeting the DoD and Army goals requires a highly complex and coordinated effort that could greatly benefit from the application of improved methods and automated analysis tools.

The U.S. Army Engineer Research and Development Center, Construction Engineering Research Laboratory (ERDC-CERL) was funded through the Army Research, Development, Test, and Evaluation (RDTE) Program to develop an intelligent framework, encompassing methodology and modeling, that describes interrelationships between energy efficiency, facility component maintenance and renewal, and mission requirements. The purpose of this framework is to facilitate the development of an integrated infrastructure investment strategy that minimizes facility total cost of ownership (TCO). A critical part of this research, and also for managers tasked with reducing energy utilization and costs, is the capability to create and apply Energy Use Intensity (EUI) benchmarks for DoD facilities. EUI represents the energy per square foot per year used by a building.

Energy utilization and cost benchmarking are very difficult for the Army because less than $0.1 \%$ of facilities are connected to the Meter Data Management System (MDMS), a central energy-reporting system operated by Huntsville District. Although the problem of energy cost and utilization data scarcity will be mitigated with the further deployment of the MDMS and policies that mandate meter deployment recently established by the Assistant Secretary of the Army (Installations, Energy and Environment), there are currently no models that accurately indicate valid EUIs for different types of Army facilities.

Commercially available benchmarks, such as the Commercial Building Energy Consumption Survey (CBECS), do not accurately capture the different types of Army facility missions, characteristics (age, construction type, 
etc.), or dynamic demand factors (troop deployment, reserve drills, etc.). In general, such benchmarking systems cannot be used by MDMS or Army managers to analyze facility consumption trends and identify poor energy performers by installation, climate zone, and other criteria.

A methodology for creating the DoD-specific EUIs would be an important part of a decision framework for applying corrective actions to facilities with the highest EUIs. Army-specific benchmarking results would allow the most effective component-renewal investment strategies by targeting the most inefficient facilities. Optimizing the timing and grouping of investments can significantly improve Army energy efficiency and reduce the TCO throughout the facility life cycle. The results of this research will help the Army to more effectively implement energy improvements, meeting or exceeding DoD energy-efficiency requirements.

\subsection{Objectives}

The objectives of this project were to

- develop integrated investment decision models

- identify EUI benchmarks that are applicable in the data-scarce Army facility-management practice

- detect and diagnose occupant-, system-, and component-level faults contributing to high facility EUIs.

\subsection{Approach}

This work addressed the development of benchmark EUIs for selected building types used on installations in the continental United States. Development of algorithms for optimizing the TCO of Army facilities focused on operational methods and requirements unique to the Army environment.

The research team evaluated data from the MDMS to understand Armywide energy consumption patterns and analyze detailed facility utility consumption data. In terms of energy consumption, the top three facilities across the Army were (1) barracks, (2) general administrative buildings, and (3) vehicle maintenance facilities. The available MDMS data for these facility types were analyzed to create benchmark EUIs for DoD-specific buildings. 


\subsection{Scope}

The text of this report may imply an assumption that installation personnel are familiar with all applicable energy use and reporting requirements, and how those requirements pertain to a wider variety of facilities. However, the authors acknowledge that installation personnel often have difficulties researching and identifying the applicability of government requirements to specific installations, facilities, and utilization cases due to competing priorities and other factors. The research team addresses this problem in a related ERDC/CERL Special Report (SR) by compiling abstracts for all applicable federal, DoD, and Army requirements and implementation guidance pertaining to facility energy metering and benchmarking. That document is published concurrently as ERDC/ CERL SR-17-13 (J osefik et al. 2017). 


\section{Meter Data Reporting Systems}

Building energy conservation efforts begin with having energy data to benchmark. Until relatively recently, DoD and the Army only had access to installation-level (also known as at-the-gate) energy-consumption data through the Defense Utility Energy Reporting System (DUERS) and Army Energy and Water Reporting System (AEWRS) systems. While these data were useful to a degree, it quickly became apparent that building-level data were required to determine the true nature of energy consumption within Army installations. Therefore, individual installations began installing meters and recording energy consumption at the building rather than at the gate. The sporadic nature of this effort, however, limited its usefulness as well. The MDMS sought to standardize the installation of these building energy meters in order to better understand Army-wide energy consumption patterns.

\subsection{Installation-level metering}

\subsubsection{Defense Utility Energy Reporting System (DUERS)}

Military installations report monthly installation-level energy use in the DUERS. The DUERS is an automated management information system with which DoD monitors its supplies and consumption of energy. It was originally fielded in February 1974 as the Defense Energy Information System (DEIS) to respond to the need to manage DoD energy resources more closely in the aftermath of the 1973 oil crisis. It is primarily used as an energy management tool, providing information about the DoD inventory and consumption of utility energy. The DUERS is used to (1) help formulate energy policy; (2) prepare management reports; (3) measure energy conservation achievements and determine progress toward energy goals and targets; (4) report energy data to Congress and other federal agencies; (5) provide online access to DoD energy data for all valid users; (6) identify energy usage and consumption trends; (7) ensure that all DoD components meet system reporting requirements; and (8) download and export energy data to automated systems for local, regional, and global analysis. ${ }^{1}$

\footnotetext{
1 DoD 5126-46-M-2, Defense Utility Energy Reporting System, November 1993, p 1-1.
} 


\subsubsection{Army Energy and Water Reporting System (AEWRS)}

All Army data submitted to DUERS is input by installations through the AEWRS. 2 This system is designed to facilitate energy management by providing timely, reliable, and accurate information on Army energy use. It provides essential energy management information to installations, regions, Major Subordinate Commands (MSCs), major Army Commands (MACOMs), Department of the Army (DA), and DoD (through DUERS). This information is used to evaluate energy trends and to determine progress toward energy use reduction goals and targets. ${ }^{3}$

\subsection{Whole-building metering}

\subsubsection{Meter Data Management System (MDMS)}

While DUERS and AWERS sought to record installation-level energy consumption, the Army Metering Program's MDMS has increased data fidelity by tracking building specific energy usage. For the past decade Army installations have been installing energy meters on their facilities in order to satisfy the energy conservation mandates discussed previously. These metering efforts, however, lacked consistency across facilities, installations, and commands. Therefore, in Fiscal Year (FY) 2007, MDMS was proposed as a means to standardize utility monitoring across all Army facilities. The FY 2008 work plan included the installation of advanced electric and natural gas meters at 22 Army installations within the continental United States and award of the MDMS software development and support contract. 4 Out-year efforts include metering program expansion to 43 installations, then to 100 more, and eventually to all installations worldwide. ${ }^{5}$

Army EXORD-028-12, dated November 2011, designated the U.S. Army Engineering and Support Center, Huntsville, Alabama (CEHNC) as the primary developer of the MDMS system. CEHNC has designed MDMS to

\footnotetext{
2 The Air Force reports via the Air Force Energy Reporting System (AFERS) and the Navy reports via the Maximo Circuits system.

3 ODUSD/I\&E, Department of Defense Energy Manager's Handbook, p 43.

4 Public Works Digest, Huntsville Center Projects Range from Saving Energy to Designing State-of-the-Art Facilities, November/December 2007, p 11.

${ }^{5}$ Advanced Metering Solutions for Federal Agencies Meeting Report, 7 December 2010. Washington Convention Center.
} 
be a secure means of collecting and analyzing metered energy data at installations. It is programmed to present the information in a graphical, web-accessed dashboard to enable rapid identification and response to energy related situations. Installation data sent to the web-based enterprise system can be used for near-real-time viewing, normalization, and analysis by installation energy managers. MDMS is intended to help them (1) manage and control installation-level energy consumption and demand, (2) act on energy-use anomalies, and (3) identify energy-saving opportunities.

At the agency level, MDMS gives the Army the data to leverage successful energy strategies across the service and highlight areas for improvement. Development of algorithms for energy use analysis using MDMS data is a primary objective of this research. The reporting status of the MDMS metering program is shown in Table 1.6

\footnotetext{
${ }^{6}$ Although MDMS was set up to retrieve meter data from Fort Leonard Wood, installation infrastructure
} was not yet available. For that reason, their meter data were acquired directly from the installation. 
Table 1. MDMS metering program status as of 18 September 2015.

\begin{tabular}{|c|c|c|c|c|c|c|}
\hline Organization & $\begin{array}{l}\text { Metered } \\
\text { Buildings }\end{array}$ & $\begin{array}{l}\text { Electric } \\
\text { Meters }\end{array}$ & $\begin{array}{c}\text { Gas } \\
\text { Meters }\end{array}$ & $\begin{array}{l}\text { Water } \\
\text { Meters }\end{array}$ & $\begin{array}{c}\text { Data } \\
\text { Interval }\end{array}$ & \begin{tabular}{|c|} 
Fully \\
Operational?
\end{tabular} \\
\hline IMCOM & 5020 & 7705 & 843 & 751 & & \\
\hline ATLANTIC REGION & 2297 & 3790 & 371 & 681 & & \\
\hline ABERDEEN PROVING GROUND & 113 & 241 & 0 & 0 & $15 \mathrm{~min}$ & $x$ \\
\hline ADELPHI LABORATORY CENTER & 14 & 106 & 3 & 0 & $15 \mathrm{~min}$ & $\mathrm{X}$ \\
\hline CARLISLE BARRACKS & 21 & 25 & 23 & 0 & $15 \mathrm{~min}$ & $\mathrm{x}$ \\
\hline FORT A P HILL & 6 & 6 & 0 & 0 & $15 \mathrm{~min}$ & $\mathrm{X}$ \\
\hline FORT BELVOIR & 252 & 787 & 0 & 0 & $30 \mathrm{~min}$ & \\
\hline FORT BENNING & 797 & 1387 & 0 & 0 & $1 \mathrm{hr}$ & \\
\hline FORT BRAGG & 589 & 496 & 207 & 678 & $15 \mathrm{~min}$ & $\underline{x}$ \\
\hline FORT CAMPBELL & 27 & 29 & 0 & 0 & $15 \mathrm{~min}$ & $\mathrm{x}$ \\
\hline FORT DETRICK & 0 & 0 & 0 & 0 & $15 \mathrm{~min}$ & \\
\hline FORT DRUM & 0 & 0 & 0 & 0 & $15 \mathrm{~min}$ & \\
\hline FORT GILLEM & 7 & 8 & 8 & 0 & $15 \mathrm{~min}$ & $\mathrm{x}$ \\
\hline FORT GORDON & 0 & 0 & 0 & 0 & $15 \mathrm{~min}$ & \\
\hline FORT HAMILTON & 0 & 0 & 0 & 0 & $15 \mathrm{~min}$ & \\
\hline FORT JACKSON & 39 & 50 & 11 & 0 & $15 \mathrm{~min}$ & $\mathrm{X}$ \\
\hline FORT KNOX & 0 & 0 & 0 & 0 & $15 \mathrm{~min}$ & \\
\hline FORT MCNAIR & 7 . & 10 & 6 & 0 & $15 \mathrm{~min}$ & $\mathrm{x}$ \\
\hline FORT MYER & 11 & 11 & 6 & 0 & $15 \mathrm{~min}$ & $\mathrm{X}$ \\
\hline FORT RUCKER & 29 & 36 & 21 & 0 & $15 \mathrm{~min}$ & $\mathrm{X}$ \\
\hline FORT STEWART & 135 & 245 & 54 & 3 & $15 \mathrm{~min}$ & \\
\hline NATICK SSC & 37 & 49 & 0 & 0 & $15 \mathrm{~min}$ & $\mathrm{x}$ \\
\hline PICATINNY ARSENAL & 98 & 153 & 0 & 0 & $1 \mathrm{hr}$ & \\
\hline REDSTONE ARSENAL & 47 & 66 & 0 & 0 & $15 \mathrm{~min}$ & $\underline{x}$ \\
\hline TOBYHANNA ARMY DEPOT & 19 & 25 & 0 & 0 & $15 \mathrm{~min}$ & $\mathrm{x}$ \\
\hline WATERVLIET ARSENAL & 0 & 0 & 0 & 0 & $15 \mathrm{~min}$ & \\
\hline WEST POINT & 49 & 60 & 32 & 0 & $15 \mathrm{~min}$ & \\
\hline CENTRAL REGION & 2054 & 3039 & 472 & 70 & & \\
\hline DETROIT ARSENAL & 16 & 35 & 7 & 0 & $15 \mathrm{~min}$ & $x$ \\
\hline DUGWAY PROVING GROUND & 19 & 24 & 0 & 0 & $15 \mathrm{~min}$ & $\mathrm{x}$ \\
\hline FORT BLISS & 188 & 244 & 124 & 67 & $15 \mathrm{~min}$ & \\
\hline FORT CARSON & 268 & 255 & 218 & 3 & $15 \mathrm{~min}$ & \\
\hline FORT HUACHUCA & 1316 & 2133 & 0 & 0 & $1 \mathrm{hr}$ & \\
\hline FORT IRWIN & 37 & 40 & 0 & 0 & $15 \mathrm{~min}$ & $\mathrm{x}$ \\
\hline FORT LEAVENWORTH & 0 & 0 & 0 & 0 & $15 \mathrm{~min}$ & \\
\hline FORT LEONARD WOOD & 0 & 0 & 0 & 0 & $15 \mathrm{~min}$ & \\
\hline FORT POLK & 26 & 26 & 7 & 0 & $15 \mathrm{~min}$ & $\mathrm{x}$ \\
\hline FORT RILEY & 0 & 0 & 0 & 0 & $15 \mathrm{~min}$ & \\
\hline FORT SILL & 0 & 0 & 0 & 0 & $15 \mathrm{~min}$ & \\
\hline JOINT BASE LEWIS-MCCHORD & 74 & 111 & 93 & 0 & $15 \mathrm{~min}$ & $\mathrm{x}$ \\
\hline PRESIDIO OF MONTEREY & 47 & 54 & 0 & 0 & $15 \mathrm{~min}$ & $\mathrm{x}$ \\
\hline ROCK ISLAND ARSENAL & 30 & 77 & 23 & 0 & $15 \mathrm{~min}$ & $\mathrm{X}$ \\
\hline WHITE SANDS MISSILE RANGE & 7 & 7 & 0 & 0 & $15 \mathrm{~min}$ & \\
\hline YAKIMA TRAINING CENTER & 0 & 0 & 0 & 0 & $15 \mathrm{~min}$ & \\
\hline YUMA PROVING GROUND & 26 & 33 & 0 & 0 & $15 \mathrm{~min}$ & $\mathrm{X}$ \\
\hline EUROPE REGION & 510 & 681 & 0 & 0 & & \\
\hline USAG BAUMHOLDER & 127 & 131 & 0 & 0 & $15 \mathrm{~min}$ & \\
\hline USAG BAVARIA & 58 & 64 & 0 & 0 & $15 \mathrm{~min}$ & \\
\hline USAG BENELUX & 9 & 11 & 0 & 0 & $15 \mathrm{~min}$ & \\
\hline USAG HEIDELBERG & 4 & 4 & 0 & 0 & $15 \mathrm{~min}$ & $\mathrm{x}$ \\
\hline USAG HOHENFELS & 20 & 23 & 0 & 0 & $15 \mathrm{~min}$ & $\mathrm{x}$ \\
\hline USAG KAISERSLAUTERN & 165 & 253 & 0 & 0 & $15 \mathrm{~min}$ & $\mathrm{x}$ \\
\hline USAG LIVORNO & 49 & 63 & 0 & 0 & $15 \mathrm{~min}$ & $\mathrm{x}$ \\
\hline USAG STUTTGART & 61 & 110 & 0 & 0 & $15 \mathrm{~min}$ & $x$ \\
\hline USAG WIESBADEN & 17 & 22 & 0 & 0 & $15 \mathrm{~min}$ & \\
\hline PACIFIC REGION & 159 & 195 & 0 & 0 & & \\
\hline FORT SHAFTER & 20 & 26 & 0 & 0 & $15 \mathrm{~min}$ & $\mathrm{X}$ \\
\hline SCHOFIELD BARRACKS & 139 & 169 & 0 & 0 & $15 \mathrm{~min}$ & $\mathrm{X}$ \\
\hline
\end{tabular}


The MDMS export files include information on location, building use, square footage, meter name, energy commodity type, timestamp, raw meter reading, units, power factor, peak demand, and average demand as shown in Table 2.

Table 2. Sample MDMS export.

\begin{tabular}{|c|c|c|c|c|c|c|c|c|c|c|}
\hline Site & Building & SF & Meter & Commodity & Timestamp & $\begin{array}{l}\text { Raw } \\
\text { reading }\end{array}$ & Units & $\begin{array}{l}\text { Power } \\
\text { factor(\%) }\end{array}$ & $\begin{array}{l}\text { Peak } \\
\text { demand }\end{array}$ & $\begin{array}{l}\text { Avg } \\
\text { demand }\end{array}$ \\
\hline FORT SAMPLE & 1234 ENLIST UPH & 123,860 & SAMP_BLDG_1234 & Electricity & $2012-09-2300: 00$ & 2055972.63 & $\mathrm{kWh}$ & NA & NA & NA \\
\hline FORT SAMPLE & 1234 ENLIST UPH & 123,860 & SAMP_BLDG_1234 & Electricity & 2012-09-23 00:15 & 2056007.38 & kWh & NA & NA & NA \\
\hline FORT SAMPLE & 1234 ENLIST UPH & 123,860 & SAMP_BLDG_1234 & Electricity & 2012-09-23 00:30 & 2056029.13 & $\mathrm{kWh}$ & NA & NA & NA \\
\hline FORT SAMPLE & 1234 ENLIST UPH & 123,860 & SAMP_BLDG_1234 & Electricity & 2012-09-23 00:45 & 2056051.63 & $\mathrm{kWh}$ & NA & NA & NA \\
\hline FORT SAMPLE & 1234 ENLIST UPH & 123,860 & SAMP_BLDG_1234 & Electricity & 2012-09-23 01:00 & 2056086.63 & kWh & NA & NA & NA \\
\hline FORT SAMPLE & 1234 ENLIST UPH & 123,860 & SAMP_BLDG_1234 & Electricity & 2012-09-23 01:15 & 2056125.00 & $\mathrm{kWh}$ & NA & NA & NA \\
\hline FORT SAMPLE & 1234 ENLIST UPH & 123,860 & SAMP_BLDG_1234 & Electricity & 2012-09-23 01:30 & 2056159.88 & kWh & NA & NA & NA \\
\hline FORT SAMPLE & 1234 ENLIST UPH & 123,860 & SAMP_BLDG_1234 & Electricity & 2012-09-23 01:45 & 2056193.88 & $\mathrm{kWh}$ & NA & NA & NA \\
\hline FORT SAMPLE & 1234 ENLIST UPH & 123,860 & SAMP_BLDG_1234 & Electricity & $2012-09-2302: 00$ & 2056230.00 & kWh & NA & NA & NA \\
\hline FORT SAMPLE & 1234 ENLIST UPH & 123,860 & SAMP_BLDG_1234 & Electricity & 2012-09-23 02:15 & 2056252.38 & $\mathrm{kWh}$ & NA & NA & NA \\
\hline FORT SAMPLE & 1234 ENLIST UPH & 123,860 & SAMP_BLDG_1234 & Electricity & $2012-09-23$ 02:30 & 2056276.25 & kWh & NA & NA & NA \\
\hline FORT SAMPLE & 1234 ENLIST UPH & 123,860 & SAMP_BLDG_1234 & Electricity & 2012-09-23 02:45 & 2056302.00 & kWh & NA & NA & NA \\
\hline FORT SAMPLE & 1234 ENLIST UPH & 123,860 & SAMP_BLDG_1234 & Electricity & 2012-09-23 03:00 & 2056323.75 & kWh & NA & NA & NA \\
\hline FORT SAMPLE & 1234 ENLIST UPH & 123,860 & SAMP_BLDG_1234 & Electricity & 2012-09-23 03:15 & 2056345.50 & $\mathrm{kWh}$ & NA & NA & NA \\
\hline FORT SAMPLE & 1234 ENLIST UPH & 123,860 & SAMP_BLDG_1234 & Electricity & 2012-09-23 03:30 & 2056368.38 & $\mathrm{kWh}$ & NA & NA & NA \\
\hline FORT SAMPLE & 1234 ENLIST UPH & 123,860 & SAMP_BLDG_1234 & Electricity & 2012-09-23 03:45 & 2056390.13 & $\mathrm{kWh}$ & NA & NA & NA \\
\hline FORT SAMPLE & 1234 ENLIST UPH & 123,860 & SAMP_BLDG_1234 & Electricity & 2012-09-23 04:00 & 2056412.88 & kWh & NA & NA & NA \\
\hline FORT SAMPLE & 1234 ENLIST UPH & 123,860 & SAMP_BLDG_1234 & Electricity & 2012-09-23 04:15 & 2056436.00 & kWh & NA & NA & NA \\
\hline FORT SAMPLE & 1234 ENLIST UPH & 123,860 & SAMP_BLDG_1234 & Electricity & $2012-09-2304: 30$ & 2056455.63 & kWh & NA & NA & NA \\
\hline FORT SAMPLE & 1234 ENLIST UPH & 123,860 & SAMP_BLDG_1234 & Electricity & 2012-09-23 04:45 & 2056478.75 & kWh & NA & NA & NA \\
\hline
\end{tabular}

\subsubsection{Commercial utility meters}

Energy meters used by private-sector utility companies and cooperatives measure data more comprehensively than those connected to the MDMS system. This is done so these businesses can balance energy production with public demand and so they can generate revenue. Private-sector utility meters typically generate reports similar to the one shown in Table 3 , with metrics for revenue, demand, power, and voltage. 
Table 3. Example report generated by a private utility provider.

\begin{tabular}{|c|c|c|c|c|c|c|c|c|c|c|c|c|c|c|c|c|}
\hline \multirow[b]{2}{*}{ Timestamp } & \multicolumn{9}{|c|}{ Revenue } & \multicolumn{2}{|c|}{ Demand } & Power & \multicolumn{4}{|c|}{ Voltage } \\
\hline & $\begin{array}{c}\text { kWh } \\
\text { del int }\end{array}$ & $\begin{array}{l}\text { kVARh } \\
\text { del int }\end{array}$ & $\begin{array}{c}\mathrm{kWh} \\
\text { rec int }\end{array}$ & $\begin{array}{l}\text { kVARh } \\
\text { rec int }\end{array}$ & $\begin{array}{c}\text { Water } \\
\text { int }\end{array}$ & $\begin{array}{l}\text { Gas } \\
\text { int }\end{array}$ & $\begin{array}{c}\text { Steam } \\
\text { int }\end{array}$ & $\begin{array}{l}\text { kWh } \\
\text { rec }\end{array}$ & $\begin{array}{c}\mathrm{kW} \text { sd } \\
\text { del }\end{array}$ & kWh del & $\begin{array}{l}\mathrm{kW} \text { sd } \\
\mathrm{mx} \text { del }\end{array}$ & PF Avg & $\begin{array}{c}\text { V1 THD } \\
\text { avg }\end{array}$ & Vln avg & I avg & $\begin{array}{c}\text { I1 Phasor } \\
\text { Angle }\end{array}$ \\
\hline 11/16/2011@13:45:00.000 & 45.49 & 3.84 & 0.00 & 0.00 & 0.00 & 0.00 & 0.00 & 0.00 & 92.66 & $179,514.64$ & 2.66 & -95.59 & 1.55 & 268.96 & 118.61 & -22.77 \\
\hline 11/16/2011@14:00:00.000 & 24.14 & 6.63 & 0.00 & 0.00 & 0.00 & 0.00 & 0.00 & 0.00 & 96.59 & $179,538.78$ & 96.59 & -96.40 & 1.58 & 273.80 & 122.67 & -20.65 \\
\hline 11/16/2011@14:15:00.000 & 24.02 & 7.13 & 0.00 & 0.00 & 0.00 & 0.00 & 0.00 & 0.00 & 96.12 & $179,562.81$ & 96.59 & -95.86 & 1.61 & 273.92 & 122.78 & -21.64 \\
\hline 11/16/2011@14:30:00.000 & 23.50 & 6.64 & 0.00 & 0.00 & 0.00 & 0.00 & 0.00 & 0.00 & 94.01 & $179,586.30$ & 96.59 & -96.23 & 1.61 & 274.12 & 119.52 & -20.42 \\
\hline 11/16/2011@14:45:00.000 & 25.18 & 8.43 & 0.00 & 0.00 & 0.00 & 0.00 & 0.00 & 0.00 & 100.77 & $179,611.48$ & 100.77 & -94.85 & 1.59 & 273.86 & 130.09 & -22.91 \\
\hline $11 / 16 / 2011$ & 22.67 & 68 & 0.00 & 0.00 & 00 & 0 & 00 & 00 & 71 & 7.66 & 77 & -95.92 & 60 & 274.64 & 115.47 & 85 \\
\hline 000 & 22.59 & 00 & 0.00 & 0.00 & 0.00 & 0.00 & .00 & 0.00 & 0.35 & $179,680.25$ & 100.77 & -95.52 & 1.59 & 94 & 5.40 & .74 \\
\hline 11/16/2011@15:45:00.000 & 23.00 & 7.46 & 0.00 & 0.00 & 0.00 & 0.00 & 0.00 & 0.00 & 92.00 & $179,703.25$ & 100.77 & -95.14 & 1.57 & 275.16 & 117.93 & -22.67 \\
\hline 11/16/2011@16:00:00.000 & 22.22 & 7.08 & 0.00 & 0.00 & 0.00 & 0.00 & 0.00 & 0.00 & 88.91 & $179,725.47$ & 100.77 & -95.28 & 1.62 & 275.51 & 113.66 & -22.63 \\
\hline 11/16/2011@16:15:00.000 & 21.19 & 6.88 & 0.00 & 0.00 & 0.00 & 0.00 & 0.00 & 0.00 & 84.77 & $179,746.66$ & 100.77 & -95.09 & 1.66 & 275.70 & 108.52 & -23.21 \\
\hline 11/16/2011@16:30:00.000 & 20.48 & 6.83 & 0.00 & 0.00 & 0.00 & 0.00 & 0.00 & 0.00 & 81.92 & $179,767.13$ & 100.77 & -94.87 & 1.55 & 275.48 & 105.10 & -22.18 \\
\hline 11/16/2011@16:45:00.000 & 18.85 & 7.37 & 0.00 & 0.00 & 0.00 & 0.00 & 0.00 & 0.00 & 75.39 & $179,785.97$ & 100.77 & -93.10 & 51 & 274.94 & 3.65 & -23.91 \\
\hline 11/16/2011@17:15:00.000 & 16.09 & 4.23 & 0.00 & 0.00 & 0.00 & 0.00 & 0.00 & 0.00 & 64.38 & $179,819.13$ & 100.77 & -96.69 & 1.58 & 273.07 & 81.94 & -18.63 \\
\hline 11/16/2011@17:30:00.000 & 15.19 & 4.16 & 0.00 & 0.00 & 0.00 & .00 & 0.00 & 0.00 & 60.77 & $179,834.31$ & 100.77 & -96.40 & 1.57 & 273.75 & 77.42 & -19.07 \\
\hline 11/16/2011@17:45:00.000 & 13.28 & 3.56 & 0.00 & 0.00 & 0.00 & 0.00 & 0.00 & 0.00 & 53.15 & $179,847.59$ & 100.77 & -96.58 & 1.60 & 274.48 & 65 & -20.96 \\
\hline 11/16/2011@18:00:00.000 & 12.33 & 3.56 & 0.00 & 0.00 & 0.00 & 0.00 & 0.00 & 0.00 & 49.35 & $179,859.92$ & 100.77 & -96.07 & 1.61 & 276.17 & 62.90 & -23.77 \\
\hline 11/16/2011@18:15:00.000 & 12.15 & 3.69 & 0.00 & 0.00 & 0.00 & 0.00 & 0.00 & 0.00 & 48.64 & $179,872.08$ & 100.77 & -95.67 & 1.55 & 276.07 & 62.28 & -24.50 \\
\hline 11/16/2011@18:30:00.000 & 12.03 & 3.67 & 0.00 & 0.00 & 0.00 & 0.00 & 0.00 & 0.00 & 48.16 & $179,884.11$ & 100.77 & -95.65 & 1.54 & 276.17 & 61.66 & -24.23 \\
\hline
\end{tabular}




\section{Building Selections}

\subsection{Priority Army building types}

A critical component of this study was the availability of detailed utility consumption data. Because Army policy requires utility metering of all buildings greater than $29,000 \mathrm{ft}^{2}$, it was assumed that such large buildings would yield the greatest amount of usable data. ${ }^{7}$ Of those buildings 29,000 $\mathrm{ft}^{2}$ and greater, the top three in combined floor area are (1) barracks, (2) general administrative buildings, and (3) vehicle maintenance facilities.

Having found the largest building types, it was quickly determined that barracks and vehicle maintenance facilities should be prioritized for their quantity and overall footprint. General administrative buildings, however, were determined to be too similar to their commercial counterparts to provide DoD-specific results. In their place, dining facilities were chosen. These buildings were generally above the $29,000 \mathrm{ft}^{2}$ threshold, and it was hypothesized that dining facilities would have higher EUIs than other building types above 29,000 $\mathrm{ft}^{2}$ in size.

Table 4. 3Q FY15 Barracks statistics in the Army real property database.

\begin{tabular}{|c|c|c|l|}
\hline $\begin{array}{c}\text { Total Floor } \\
\text { Area (ft }{ }^{2} \text { ) }\end{array}$ & $\begin{array}{c}\text { Quantity } \\
\text { (Q3 FY15) }\end{array}$ & CATCODE & \multicolumn{1}{|c|}{ Description } \\
\hline $7,448,548$ & 97 & 72010 & Transient Lodging \\
\hline $53,336,692$ & 978 & 72111 & Enlisted Unaccompanied Personnel Housing \\
\hline $2,401,052$ & 27 & 72112 & Unaccompanied Housing for Wounded Warriors \\
\hline $4,948,617$ & 121 & 72114 & Annual Training/Mobilization Barracks (TT/ENL) \\
\hline 301,284 & 7 & 72115 & Annual Training/Mobilization Barracks (MOB ENL BRKS) \\
\hline $5,653,690$ & 82 & 72121 & Student Barracks (TRANS UPH/AIT) \\
\hline $3,258,300$ & 60 & 72122 & Student Barracks (TRANS UPH/AST) \\
\hline 917,580 & 21 & 72170 & Enlisted Unaccompanied Personnel Housing (UPH SR NCO) \\
\hline $9,423,078$ & 145 & 72181 & Recruit/Trainee Barracks \\
\hline $1,693,337$ & 38 & 72410 & Officer Unaccompanied Personnel Housing \\
\hline $\mathbf{8 9 , 3 8 2 , 1 7 8}$ & $\mathbf{1 , 5 7 6}$ & & \multicolumn{2}{|l}{ Total } \\
\hline
\end{tabular}

\footnotetext{
7 Valine, Debra, "Corps of Engineers helps Army installations reduce energy use, save money", USACE Huntsville Center, August 31, 2009, http://www.army.mil/mobile/article/?p=26770
} 
Table 5. 3QFY15 Vehicle maintenance shop statistics in the Army real property database.

\begin{tabular}{|c|c|c|l|}
\hline $\begin{array}{c}\text { Total Floor } \\
\text { Area (ft }{ }^{2} \text { ) }\end{array}$ & $\begin{array}{c}\text { Quantity } \\
\text { (Q3 FY15) }\end{array}$ & CATCODE & \multicolumn{1}{|c|}{ Description } \\
\hline $1,801,249$ & 41 & 21407 & Vehicle Maintenance Shop, National Guard (ARNG VEH MAINT) \\
\hline 497,208 & 13 & 21409 & Vehicle Maintenance Shop, Reserve (USAR VEH MAINT) \\
\hline $10,310,212$ & 226 & 21410 & Vehicle Maintenance Shop \\
\hline 142,969 & 3 & 21412 & Vehicle Maintenance Shop (MAINT STORAGE) \\
\hline 135,104 & 3 & 21414 & Vehicle Maintenance Shop (GEN ITEM REPAIR) \\
\hline 30,084 & 1 & 21416 & Vehicle Maintenance Shop (MSL MAINT FAC) \\
\hline 103,881 & 2 & 21417 & Vehicle Maintenance Shop (VEH PNT/PREP SH) \\
\hline 408,273 & 10 & 21418 & Vehicle Maintenance Shop, Reserve (AMSA/ECS) \\
\hline $5,346,017$ & 78 & 21419 & Vehicle Maintenance Shop, National Guard (CSMS/MATES) \\
\hline $2,044,397$ & 24 & 21435 & Vehicle Maintenance Shop, Depot (MAJ END ITM REB) \\
\hline $2,407,568$ & 25 & 21440 & Vehicle Maintenance Shop, Depot (COMP REB DEPOT) \\
\hline $\mathbf{2 3 , 2 2 6 , 9 6 2}$ & $\mathbf{4 2 6}$ & & \multicolumn{2}{|l}{} \\
\hline & & & Total \\
\hline
\end{tabular}

Table 6. 3QFY15 Dining facility statistics in the Army Real Property database.

\begin{tabular}{|c|c|c|lc|}
\hline $\begin{array}{c}\text { Total Floor } \\
\text { Area } \text { (ft }^{2} \text { ) }\end{array}$ & $\begin{array}{c}\text { Quantity } \\
\text { (Q3 FY15) }\end{array}$ & CATCODE & & Description \\
\hline $1,360,446$ & 30 & 72210 & Dining Facility \\
\hline 67,791 & 2 & 72212 & Dining Facility \\
\hline $\mathbf{1 , 4 2 8 , 2 3 7}$ & $\mathbf{3 2}$ & & & Total \\
\hline
\end{tabular}

\subsection{Building attributes}

\subsubsection{EISA-required building attributes}

Energy Independence and Security Act (EISA), Section 432 IIC, specifies the minimum number of building attributes to be used in energy benchmarking. These attributes allow for reasonable comparisons between similar buildings:

- Building name or identifier

- Building type according to use

- Building location by climate zone ${ }^{8}$ or zip code (see Figure 1 )

- Floor area (gross square feet) 
Figure 1. International Energy Conservation Code (IECC) climate zone map.

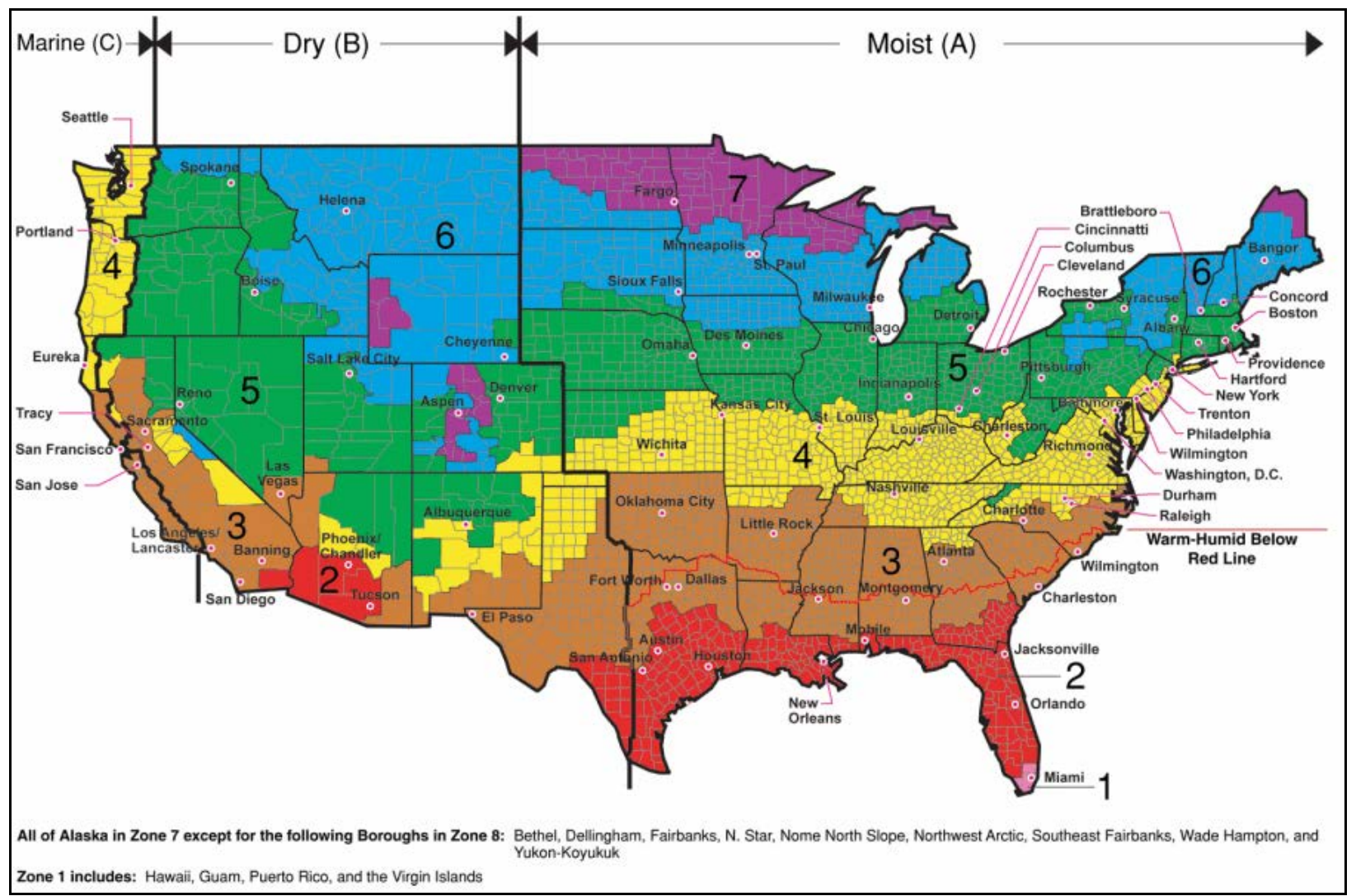

Since more attributes tend to yield increasingly more accurate benchmarks, additional attributes from the Army real property database were considered (see section 3.2.2).

\subsubsection{Building attributes in Army's HQIIS}

The Headquarters Installation Information System (HQIIS) is the official registry of Army installations and sites, and the data warehouse for Army real property and related information. The system interfaces with the DoD real property asset registry for statutory and regulatory real property reporting. The primary source systems from which HQIIS receives real property data are:

- GFEBS (General Fund Enterprise Business System),

- PRIDE (Planning Resource Infrastructure Development and Evaluation System),

- RFMIS (Rental Facility Management Information System), and

- REMIS (Real Estate Management Information System). 
The project-relevant building attributes represented by data fields in HQIIS are shown in Table 7.

Table 7. Energy-relevant building attributes in HQIIS.

\begin{tabular}{|l|l|}
\hline Installation name & Foundation construction material \\
\hline Facility number & Structural construction material \\
\hline Facility name & Roof construction material \\
\hline Built date & Floor construction material \\
\hline Current category code/description & Wall construction material \\
\hline Design use category code/description & Heating type \\
\hline Special quantities (meals served, beds, etc.) & Heating fuel \\
\hline Total area & Cooling type \\
\hline Floors below ground & Air circulation type \\
\hline Floors above ground & Advanced electric meter \\
\hline Floor-to-ceiling height & Advanced gas meter \\
\hline Construction material & Advanced water meter \\
\hline
\end{tabular}

In HQIIS, each of the attributes above is linked to a pick list from which to select an input to associate with a facility. The attribute inputs are used in the benchmarking process so system limits to attribute input choices can curtail benchmarking activities. For example, HQIIS does not track window-to-wall ratios, assembly U-Factors, the presence or absence of sustainability features (e.g., cool roofs), and emerging energy technologies (e.g. wind, hydrogen, or geothermal power), all of which are useful for a thorough benchmarking analysis. While this analysis is complete and utilizes all the available data, with these additional attributes, the benchmarks could have been even more accurate.

\subsubsection{DoD data analytics and integration support requirements}

DoD is subject to extensive energy reporting requirements that impose a growing burden on DoD personnel to collect, analyze, package, and transmit energy data. DoD currently does not have standardized enterprise facility energy information, nor does it have enterprise-wide information technology systems to support facility energy management. This lack of standardization requires energy managers and other personnel to manually collect, transform, and reformat data to meet separate data calls. This situation greatly reduces efficiency and leaves energy managers with little 
time to manage facility energy consumption, or to focus on ways to improve facility energy efficiency.

The Data Analytics and Integration Support (DAIS) platform supports OSD (I\&E) Business Enterprise Integration office Real Property Inventory Reporting (RPIR) and Enterprise Energy Information Management (EEIM) requirements. EEIM building attributes are being established based on those used by ENERGY STAR® Portfolio Manager.

For comparison, available Army and DoD building attributes can be evaluated against those used by ENERGY STAR ${ }^{\circledR}$ Portfolio Manager (Table 8).

Table 8. EPA Portfolio Manager building attributes by facility type (EEIM).

\begin{tabular}{|c|c|c|c|c|c|c|c|c|c|c|}
\hline \multirow[b]{2}{*}{ Required Information } & \multicolumn{10}{|c|}{ Facility Type } \\
\hline & Office & Education & \begin{tabular}{|l|} 
Food \\
Sales
\end{tabular} & Mercantile & $\begin{array}{c}\text { Warehouse } \\
\text { \& Storage }\end{array}$ & \begin{tabular}{|c|} 
Lodging - \\
Residential \\
\end{tabular} & $\begin{array}{l}\text { Lodging - } \\
\text { Hoteling }\end{array}$ & Hospital & $\begin{array}{l}\text { Religious } \\
\text { Worship }\end{array}$ & Parking \\
\hline Gross Square Feet & $\checkmark$ & $\checkmark$ & $\checkmark$ & $\checkmark$ & $\checkmark$ & $\checkmark$ & $\checkmark$ & $\checkmark$ & $\checkmark$ & \\
\hline Weekly Operating Hours & $\checkmark$ & & $\checkmark$ & $\checkmark$ & $\checkmark$ & & & & $\checkmark$ & $\checkmark$ \\
\hline No. of workers on main shift & $\checkmark$ & & $\checkmark$ & $\checkmark$ & $\checkmark$ & & $\checkmark$ & & & \\
\hline No. of personal computers/registers & $\checkmark$ & $\checkmark$ & & $\checkmark$ & & & & & $\checkmark$ & \\
\hline$\%$ of floor area airconditioned $(>=50 \%,<50 \%$, or none) & $\checkmark$ & & & & & & & & & \\
\hline$\%$ of floor area heated $(>=50 \%,<50 \%$, or none) & $\checkmark$ & & & & & & & & & \\
\hline No. of licensed beds & & & & & & & & $\checkmark$ & & \\
\hline No. of floors & & & & & & $\checkmark$ & & $\checkmark$ & & \\
\hline Tertiary care facility (yes/no) & & & & & & & & $\checkmark$ & & \\
\hline Lab on-site (yes/no) & & & & & & & & $\checkmark$ & & \\
\hline Laundry facilities on-site (yes/no) & & & & & & & & $\checkmark$ & & \\
\hline No. of buildings & & & & & & & & $\checkmark$ & & \\
\hline On-site cooking (yes/no) & & $\checkmark$ & $\checkmark$ & & & & $\checkmark$ & & $\checkmark$ & \\
\hline No. walk-in refrigeration/freezer units & & $\checkmark$ & $\checkmark$ & $\checkmark$ & & & $\checkmark$ & & $\checkmark$ & \\
\hline$\%$ of floor area cooled in $10 \%$ increments $(10 \%, 20 \%, 30 \%$,etc.) & & $\checkmark$ & $\checkmark$ & $\checkmark$ & $\checkmark$ & $\checkmark$ & $\checkmark$ & & & \\
\hline$\%$ of floor area heated in $10 \%$ increments $(10 \%, 20 \%, 30 \%$,etc. $)$ & & $\checkmark$ & $\checkmark$ & $\checkmark$ & $\checkmark$ & $\checkmark$ & $\checkmark$ & & & \\
\hline \# open or closed refrigeration/freezer cases & & & $\checkmark$ & $\checkmark$ & & & & & & \\
\hline Days of operation & & $\checkmark$ & & & & & & & $\checkmark$ & \\
\hline High School (yes/no) & & $\checkmark$ & & & & & & & & \\
\hline Months of use & & $\checkmark$ & & & & & & & & \\
\hline Maximum seating capacity & & & & & & & & & $\checkmark$ & \\
\hline No. of units & & & & & & $\checkmark$ & & & & \\
\hline No. of bedrooms & & & & & & $\checkmark$ & & & & \\
\hline No. laundry hookups in each unit & & & & & & $\checkmark$ & & & & \\
\hline No of diswashers in each unit & & & & & & $\checkmark$ & & & & \\
\hline Affordable or market rate & & & & & & $\checkmark$ & & & & \\
\hline Hrs/day guests on-site & & & & & & & $\checkmark$ & & & \\
\hline No guest meals served & & & & & & & $\checkmark$ & & & \\
\hline Sq ft - full service spas & & & & & & & $\checkmark$ & & & \\
\hline Sq ft - gym/fitness center & & & & & & & $\checkmark$ & & & \\
\hline Annual qty of laundry processed on-site & & & & & & & $\checkmark$ & & & \\
\hline Average occupany (\%) & & & & & & & $\checkmark$ & & & \\
\hline Gross Square Feet enclosed & & & & & & & & & & $\checkmark$ \\
\hline Gross Square Feet not enclosed with roof & & & & & & & & & & $\checkmark$ \\
\hline Gross Square Feet open & & & & & & & & & & $\checkmark$ \\
\hline
\end{tabular}




\section{Analysis and Results}

\subsection{Data collection}

This study sought to establish benchmark energy usage intensities for certain building types within domestic army installations. Specifically, barracks, dining facilities (DFACs), and vehicle maintenance (Veh. Maint) buildings were analyzed. The data were primarily obtained using the MDMS database. However, data for all buildings in American Society of Heating, Refrigerating and Air Conditioning Engineers (ASHRAE) Climate Zone 4A were obtained directly from the utility provider.

After scouring the MDMS database and utility data for all buildings of the desired types at installations throughout the country, 115 examples were found. From these initial 115 buildings, 71 were determined to be acceptable candidates for this study. The remaining 44 buildings were eliminated from the final analysis due to the following factors:

- Lack of one continuous year's worth of data

- Intermittent data

- Missing either gas or electric data

- Obvious errors in MDMS data

- Questionable validity of data

The building type composition of the 71 analyzed buildings can be seen in Figure 2, the location of the analyzed buildings can be seen in Figure 3, and the construction types can be seen in Figure 4. These buildings ranged between 254,000 $\mathrm{ft}^{2}$ and 6,435 $\mathrm{ft}^{2}$ and were built between 1927 and 2012. A detailed breakdown by attribute of each building can be found in Table A1, Table A2, and Table A3 in the Appendix. 
Figure 2. Building use types.

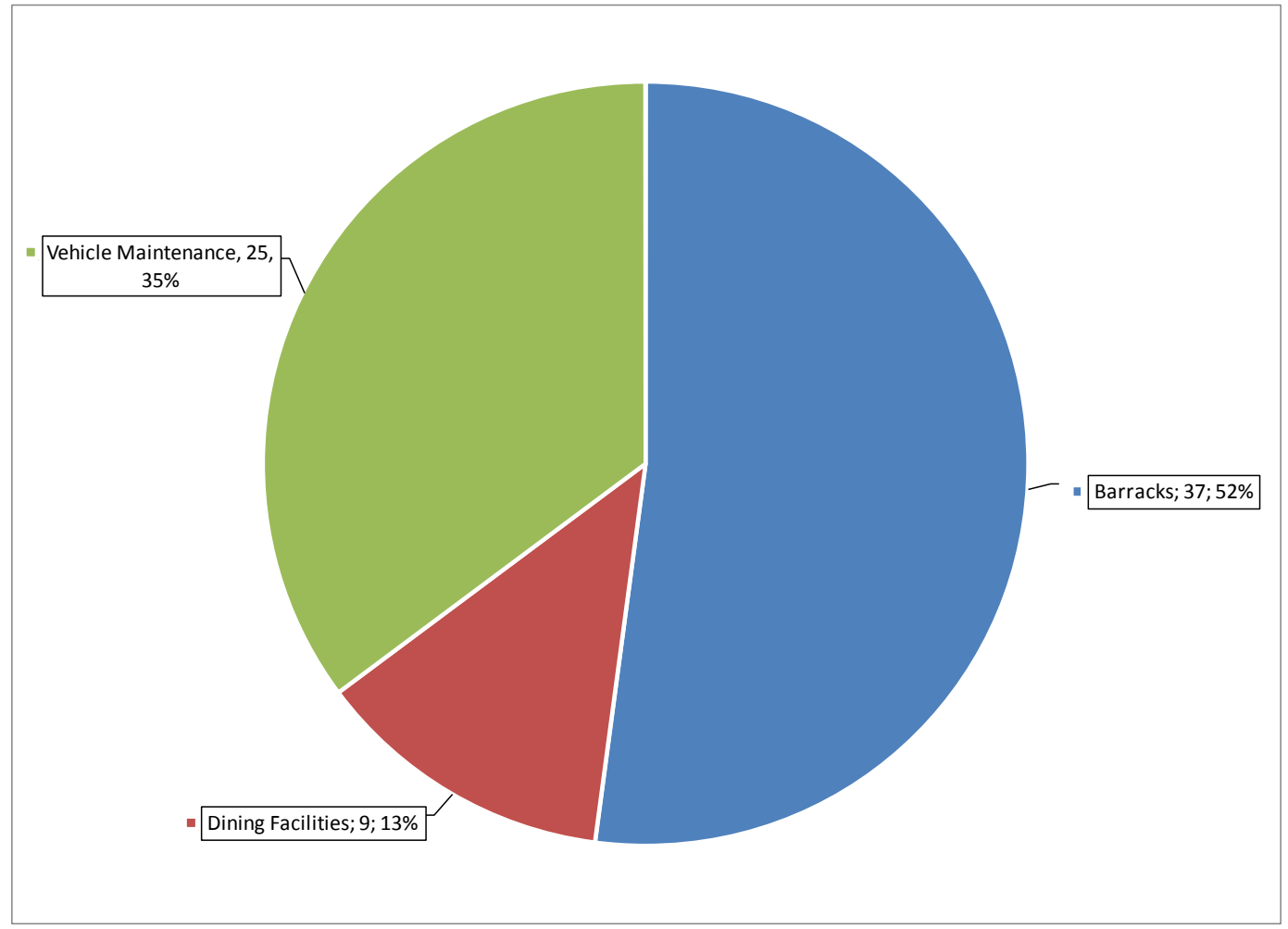

Figure 3. Building climate zone locations.

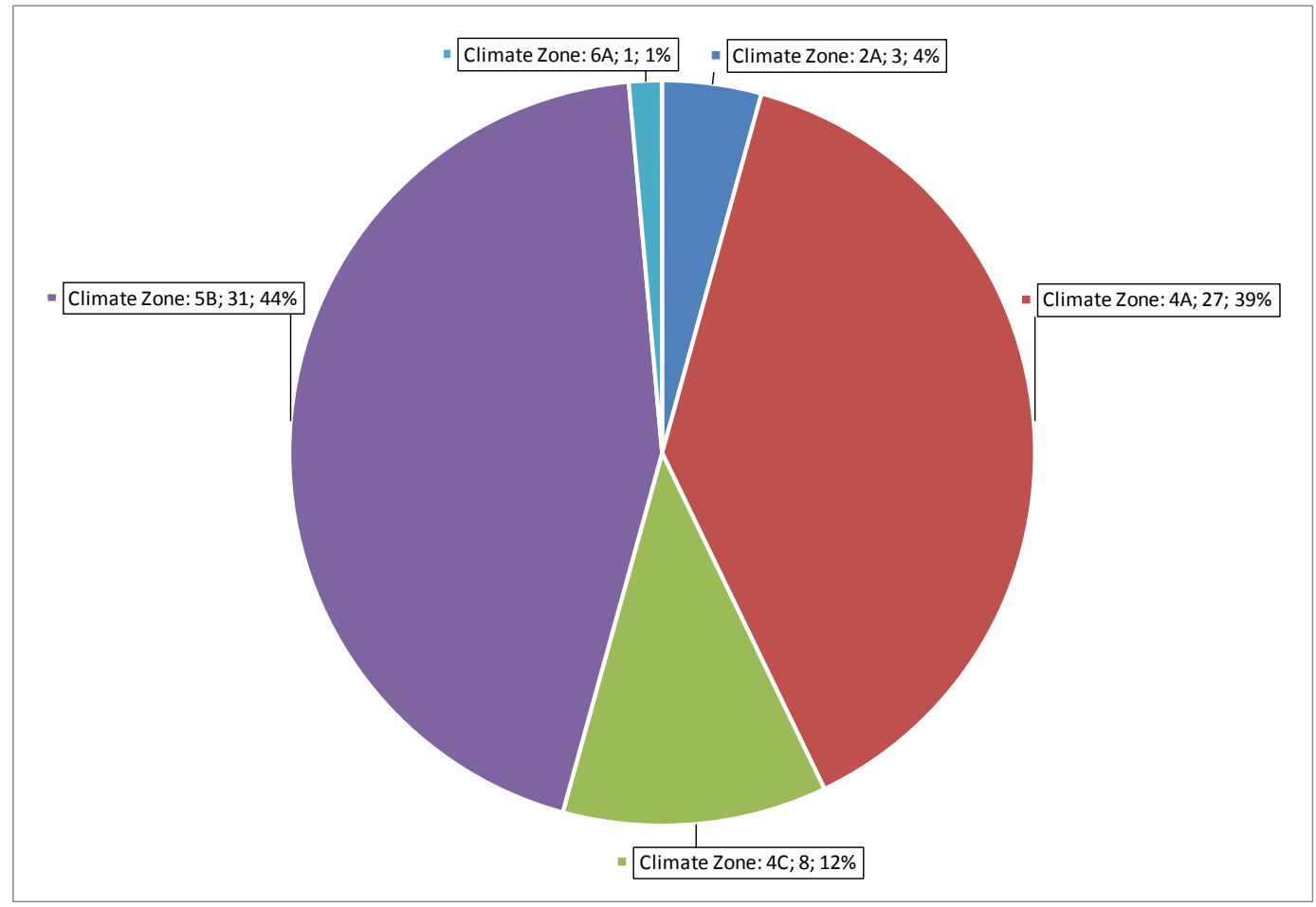


Figure 4. Building construction types.

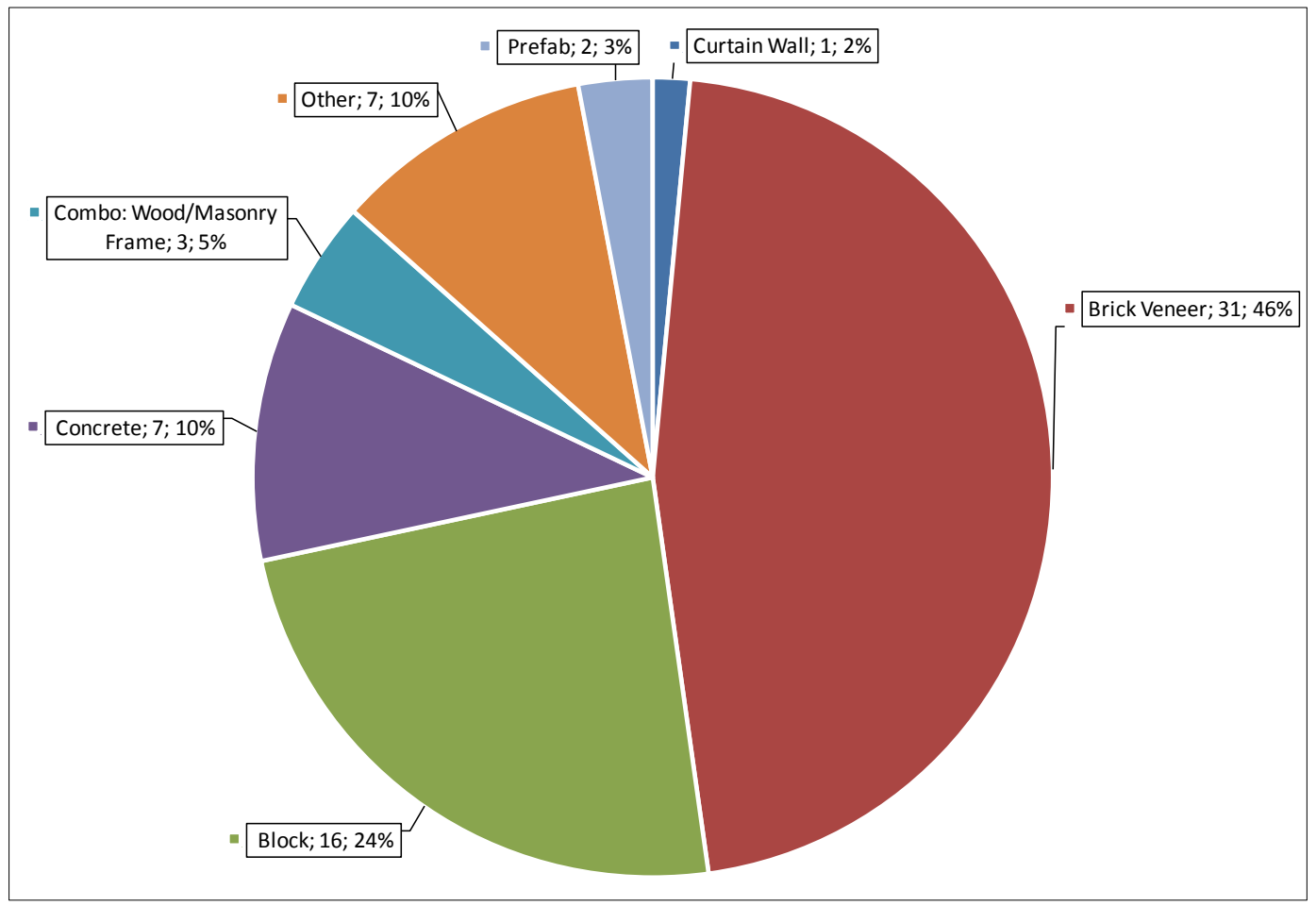

\subsection{Insufficient data}

Data scarcity proved to be the primary challenge for establishing the desired benchmarks. As stated above, MDMS was the main source for the analyzed data. The database was queried for barracks, vehicle maintenance buildings, and dining facilities using gas and/ or electric fuel sources with data recorded in 15-minute increments. With such high resolution data, it should have been possible to establish accurate and detailed energy profiles.

Unfortunately, due to technological and logistical challenges, the MDMS database remains sparsely populated. Many buildings and installations do not yet have the equipment or manpower to comply with the MDMS standard. Moreover, many installations have yet to implement or have only recently implemented utility metering to the degree necessary for such a study. Electric meters have only been required since October 1, 2012, according to Section 103 of Energy Policy Act of 2005 (EPAct 2005) ${ }^{9}$ and gas meters will be required at all installations by October 1, 2015, according to the Energy Independence and Security Act of 2007 (EISA 
2007). ${ }^{10}$ Therefore, the required annual data were only available for a handful of installations and buildings at the time of this study. Additionally, although data were available for Climate Zone 4A buildings, only electric was recorded in 15-minute increments. Gas data were provided on a monthly basis and recorded hourly.

\subsection{Data scrubbing}

Although most data were obtained directly from MDMS, the data required significant refinement before use. Most likely because Army installations and their respective energy managers are individually responsible for all MDMS data, the downloaded energy consumption figures proved to be of poor and inconsistent quality. Without oversight from MDMS administrators or a means to standardize the information provided to MDMS, energy data are bound to vary between these installations. Therefore, to achieve the uniformity required for a thorough analysis, the downloaded MDMS data were "scrubbed" with a tool developed with Microsoft Excel Visual Basic for Applications (VBA). An example screen of this data-scrubbing tool can be seen in Figure 5, and functions are listed in Table 9. Depending on the type of data errors, various combinations of data-scrubbing routines were applied to yield data appropriate for use in benchmarking. 
Figure 5. The 'datacleanup' tab of the Excel tool.

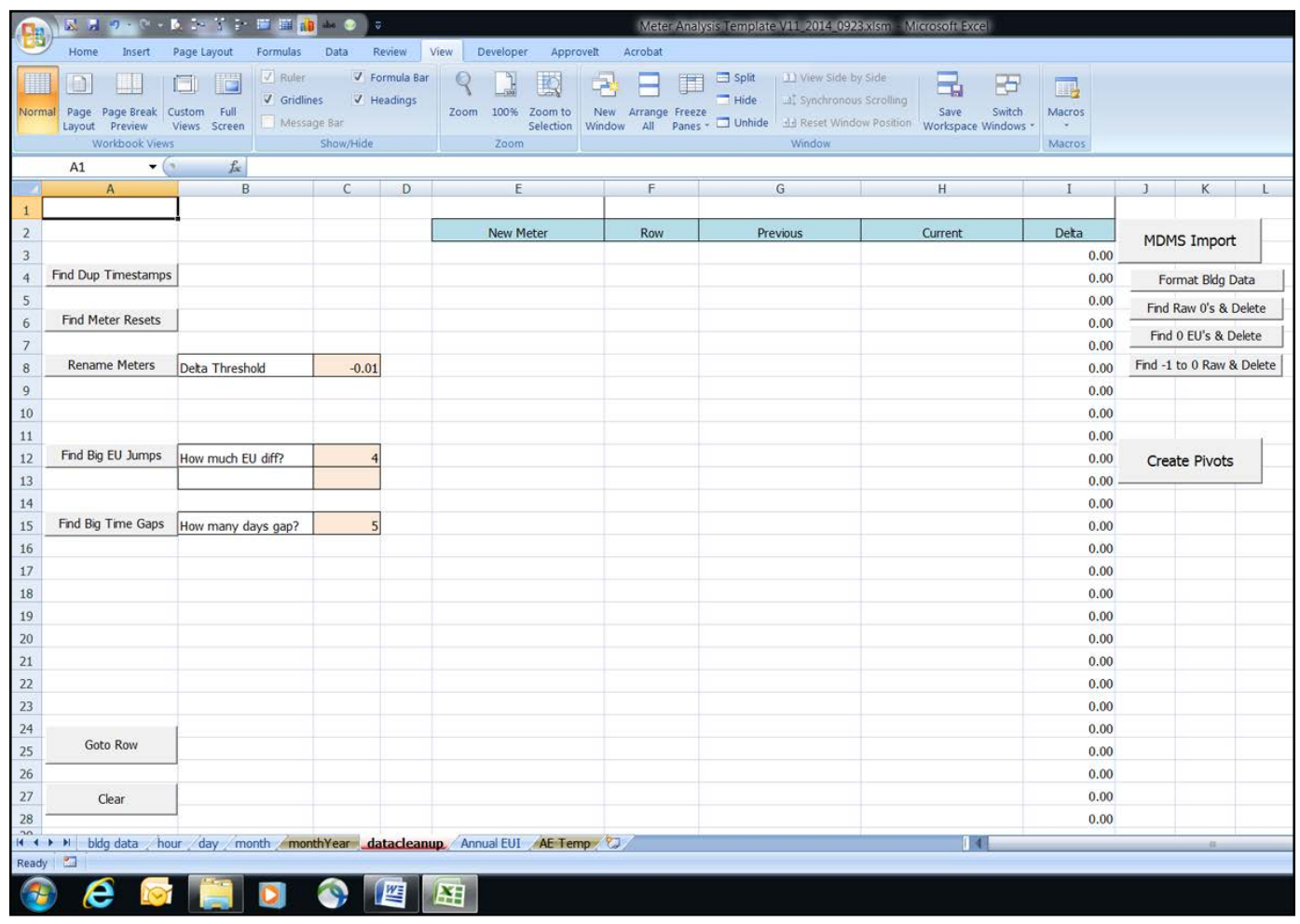

Table 9. Functions in Excel data scrubbing tool.

\begin{tabular}{|c|c|c|}
\hline Button & Action & Formatting \\
\hline $\begin{array}{l}\text { Find Dup } \\
\text { Timestamps }\end{array}$ & $\begin{array}{l}\text { Finds data entries in 'bldg data' worksheet with same date } \\
\text { and time (e.g. those related to Daylight Savings Time), or finds } \\
\text { first entry of new meter. }\end{array}$ & $\begin{array}{l}\text { highlights duplicate timestamps } \\
\text { orange; highlights meter name } \\
\text { changes red }\end{array}$ \\
\hline $\begin{array}{l}\text { Find Meter } \\
\text { Resets }\end{array}$ & $\begin{array}{l}\text { Finds net negative energy usage entries in 'bldg data' } \\
\text { worksheet. }\end{array}$ & $\begin{array}{l}\text { highlights negative energy usage } \\
\text { orange; highlights meter name } \\
\text { changes red }\end{array}$ \\
\hline Rename Meters & $\begin{array}{l}\text { Used to change meter names for legitimate meter resets (e.g. } \\
\text { power outages, maximum meter digits exceeded, etc.); } \\
\text { requires that 'Find Meter Resets' results be displayed. }\end{array}$ & $\begin{array}{l}\text { appends meter name with } 01,02, \\
\text { etc.; highlights meter name changes } \\
\text { red }\end{array}$ \\
\hline $\begin{array}{l}\text { Find Big EU } \\
\text { Jumps }\end{array}$ & $\begin{array}{l}\text { Finds specified increase in energy usage between rows in } \\
\text { 'bldg data' worksheet. }\end{array}$ & $\begin{array}{l}\text { highlights big EU jumps orange; } \\
\text { highlights meter name changes red }\end{array}$ \\
\hline $\begin{array}{l}\text { Find Big Time } \\
\text { Gaps }\end{array}$ & Finds specified time gap in 'bldg data' worksheet. & $\begin{array}{l}\text { highlights big time gaps orange; } \\
\text { highlights meter name changes red }\end{array}$ \\
\hline Goto Row & $\begin{array}{l}\text { Goes to row on 'bldg data' worksheet when row is selected on } \\
\text { the 'datacleanup' results table. }\end{array}$ & $n / a$ \\
\hline Clear & $\begin{array}{l}\text { Clears table data on the 'datacleanup' worksheet and clears } \\
\text { row highlighting on the 'bldg data' worksheet. }\end{array}$ & $n / a$ \\
\hline MDMS Import & $\begin{array}{l}\text { Imports and formats data from open 'MDMS data file' into } \\
\text { 'bldg data' worksheet. }\end{array}$ & $\begin{array}{l}\text { formats fonts, alignment, and borders; } \\
\text { autosizes columns; and sorts data by } \\
\text { meter then timestamp }\end{array}$ \\
\hline $\begin{array}{l}\text { Format Bldg } \\
\text { Data }\end{array}$ & $\begin{array}{l}\text { Performs MDMS Import button formatting function without } \\
\text { importing data. }\end{array}$ & $\begin{array}{l}\text { formats fonts, alignment, and borders; } \\
\text { autosizes columns; and sorts data by } \\
\text { meter then timestamp }\end{array}$ \\
\hline
\end{tabular}




\begin{tabular}{|l|l|l|}
\hline Button & Action & Formatting \\
\hline $\begin{array}{l}\text { Find Raw 0s \& } \\
\text { Delete }\end{array}$ & Finds and deletes all rows with meter read values of zero (0). & n/a \\
\hline $\begin{array}{l}\text { Find 0 EUs \& } \\
\text { Delete }\end{array}$ & $\begin{array}{l}\text { Finds raw usage readings that are identical for two or more } \\
\text { consecutive rows and deletes subsequent rows (i.e. rows with } \\
\text { no net energy consumption). }\end{array}$ & n/a \\
\hline $\begin{array}{l}\text { Find -1 to 0 Raw } \\
\text { \& Delete }\end{array}$ & $\begin{array}{l}\text { Finds and deletes all rows with miniscule (i.e. negligible) } \\
\text { negative meter read values. }\end{array}$ & n/a \\
\hline Create Pivots & $\begin{array}{l}\text { Analyzes 'bldg data' worksheet and populates the 'hour', } \\
\text { 'day', 'month', 'monthYear', and 'Annual EUl', worksheets with } \\
\text { the analyzed data. }\end{array}$ & n/a \\
\hline
\end{tabular}

\subsection{Data selection}

From this dataset, 115 buildings demonstrated the requisite characteristics. Upon further analysis, however, 44 of those buildings were found to contain insufficient data to establish clear benchmarks. The remaining buildings consisted of 37 barracks, 25 vehicle maintenance buildings, and 9 dining facilities (see Appendix for details).

Only the most recent, continuous, yearlong data were considered for the final 71 buildings. This implied that all buildings were not analyzed for the same time period. The analysis period is specified for each building in Table A1, Table A2, and Table A3 in the Appendix. Within those tables, both electricity and gas consumption are recorded in kBTUs and EUIs are expressed as annual $\mathrm{kBTU} / \mathrm{ft}^{2}$ used by the building rather than delivered by the source. Project resources did not allow for weather-normalizing of the data or normalizing for building occupancy or renovations. Also, due to the absence of sub-metering, the data do not distinguish building loads from process loads.

After finalizing the building dataset based upon the parameters explained above, the data were statistically analyzed to determine the presence of outliers. First, a Shapiro-Wilk test was performed to determine the normality of the each building type dataset. As can be seen in Table 10, both barracks and DFACs were relatively normally distributed assuming an $\alpha$ statistic of 0.05 . Once normality was established, a Grubbs test was conducted for each building type to determine if an outlier was present within the dataset. The results of the Grubbs test are presented in Table 11 and show that no outliers within the three separate building type datasets. The potential outliers are highlighted in Table A1, Table A2, and Table A3 (see Appendix). Although vehicle maintenance buildings did not fall within a 
normal distribution according to the Shapiro-Wilk test, the maximum variation from the mean for this data set was 1.72 standard deviations, which is well below the three standard deviations commonly used to consider a data point an outlier.

Table 10. Shapiro-Wilk test for normality.

\begin{tabular}{|c|r|r|r|}
\hline & Barracks & Dining Facilities & Vehicle Maintenance \\
\hline $\boldsymbol{\alpha}$ & 0.05 & 0.05 & 0.05 \\
\hline $\mathbf{W}$ & 0.96 & 0.91 & 0.90 \\
\hline $\mathbf{p}$ & 0.17 & 0.31 & 0.02 \\
\hline Normality $(\mathbf{p}>\boldsymbol{\alpha})$ ? & Yes & Yes & No \\
\hline
\end{tabular}

Table 11. Grubbs test for outliers.

\begin{tabular}{|c|c|c|c|}
\hline & Barracks & Dining Facilities & Vehicle Maintenance \\
\hline Potential Outlier & 219.25 (Building B23) & 577.14 Building (D4) & 172.49 (Building V4) \\
\hline G & 2.68 & 1.55 & 1.72 \\
\hline G-crit & 2.84 & 2.11 & 2.66 \\
\hline Significant & No & No & No \\
\hline
\end{tabular}

Having removed all erroneous data from the initial MDMS dataset, and after checking for outliers, the remaining 71 buildings were analyzed to establish the final benchmarking guidelines. Within the analysis several trends were investigated to determine which variables are most relevant for building energy performance. Although the data may not be consistent with all Army buildings, one can find interesting energy insights and determine baselines for future analysis. The following section discusses the results of the data analysis.

\subsubsection{Estimating missing data-Microsoft Excel LINEST function}

Upon analyzing the gas and electric meter data for barracks, dining halls, and vehicle maintenance facilities at various installations, it became apparent that several installations lacked utility data for certain months in the year. Although the cause of the missing data is unknown, such data could not be used to calculate accurate EUIs. Therefore, two attempts were made to estimate the missing values. Both techniques proved to be inadequate at capturing the data as accurately as desired.

The first attempted method used the line statistics (LINEST) function built into Microsoft Excel. This function uses the least-squares method to calculate the line that best fits the available data. For this exercise, the function 
was applied to calculate the equation of a 6th degree polynomial from the available meter data. The missing months were then calculated using this computed equation. Although energy usage does not follow a particular function, the 6th degree polynomial could approximate the values with the greatest accuracy. When a month was removed from a complete data set, the LINEST regression was able to calculate the missing data to within $1.1 \%$ of the actual value. The specific values can be seen in Table 12 below and the regressions can be compared in Figure 6.

Table 12. Actual vs. estimated energy usage (1 month, LINSET function, Building 1).

\begin{tabular}{|c|c|c|c|c|c|c|c|c|c|c|c|c|}
\hline \multicolumn{3}{|c|}{ nergy Usage (Actual) } & \multirow[b]{2}{*}{3} & \multirow[b]{2}{*}{3} & \multirow[b]{2}{*}{5} & \multirow[b]{2}{*}{6} & \multirow[b]{2}{*}{7 (Actual) } & \multirow{3}{*}{\begin{tabular}{|r|}
8 \\
98015.49
\end{tabular}} & \multirow{3}{*}{$\begin{array}{r}9 \\
78192.00\end{array}$} & \multirow{3}{*}{\begin{tabular}{|r}
10 \\
62777.24 \\
\end{tabular}} & \multirow{3}{*}{\begin{tabular}{|r}
11 \\
78956.97 \\
\end{tabular}} & \multirow{3}{*}{\begin{tabular}{|r}
12 \\
125382.57
\end{tabular}} \\
\hline Month & & 2 & & & & & & & & & & \\
\hline Energy Usag & 2001.31 & 89019.32 & .13 & 99.22 & 3.78 & 86.49 & 77.90 & & & & & \\
\hline & Usage (Estin & & & & & & & & & & & \\
\hline inth & 7 & & 3 & & & & & & 9 & 100 & 11 & \\
\hline Energy Usą & 102001.31 & 89019.32 & 7795.13 & 62179.22 & 81803.78 & 88786.49 & 100167.53 & 98015.49 & 78192.00 & 62777.24 & 78956.97 & 125382 \\
\hline ercenta & $0.00 \%$ & $0.00 \%$ & $0.00 \%$ & $0.00 \%$ & $0.00 \%$ & $0.00 \%$ & $1.10 \%$ & $0.00 \%$ & $0.00 \%$ & $0.00 \%$ & $0.00 \%$ & ti \\
\hline
\end{tabular}

Figure 6. Building 1 actual data also highlighting one estimated month.

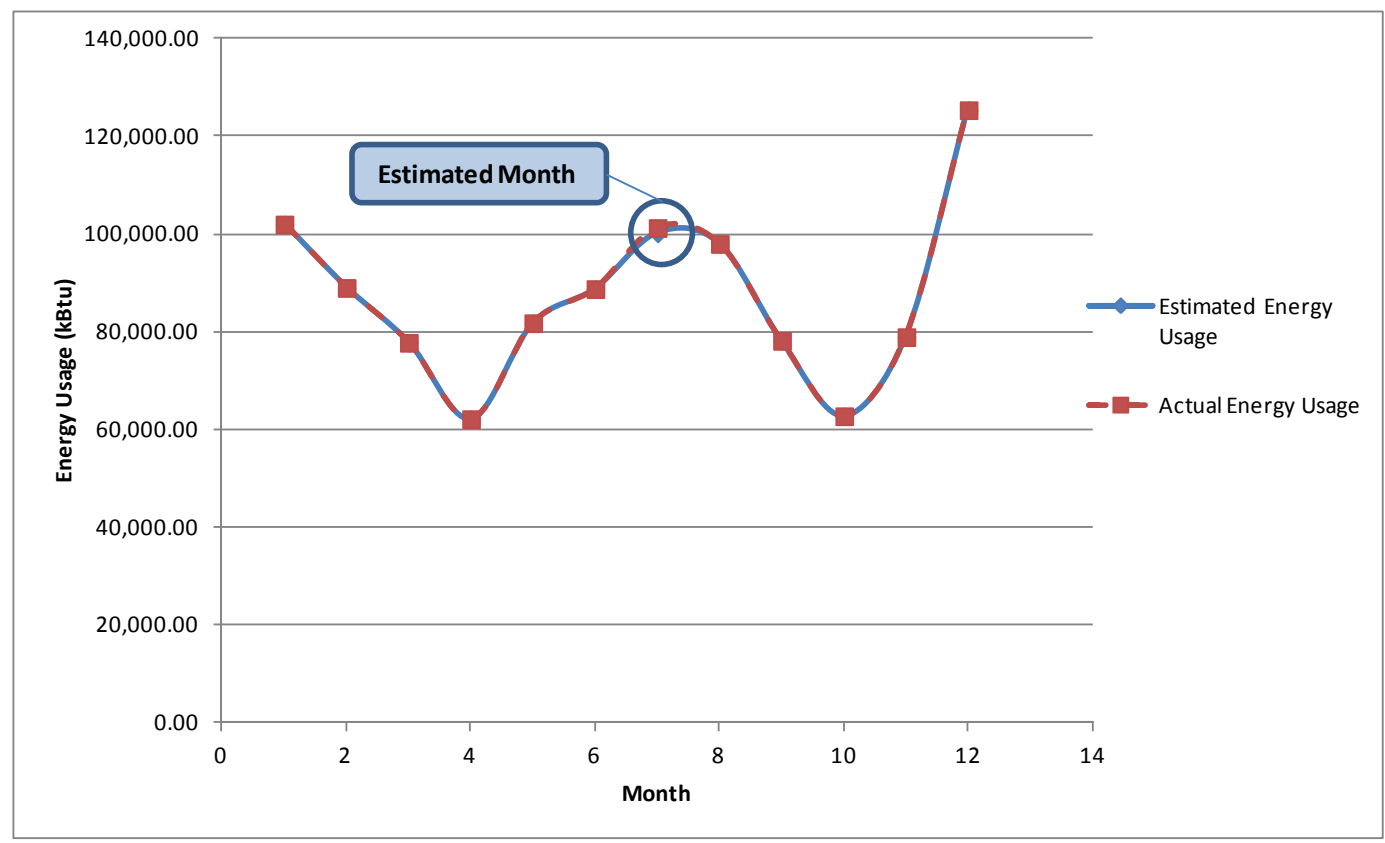

Although this method produced satisfactory results in this example, it must be noted that this regression cannot guarantee exact results. If energy usage were to increase or decrease suddenly for the missing months, the trend would not be captured by the LINEST function. Data missing at the end or the beginning of the series also cannot be estimated using this method and large gaps in the data yield inaccurate results, as can be seen in Table 13 and Figure 7. Since most buildings with limited data lacked energy consumption figures for multiple and/ or consecutive months, this 
method could not estimate the missing data with a reasonable amount of accuracy.

Table 13. Actual vs. estimated energy usage (3 months, LINSET function, Building 1).

\begin{tabular}{|c|c|c|c|c|c|c|c|c|c|c|c|c|}
\hline \multicolumn{13}{|c|}{ Electric Energy Usage (Actual) } \\
\hline Month & 1 & 2 & 3 & 4 & 5 (Actual) & 6 (Actual) & 7 (Actual) & 8 & 9 & 10 & 11 & 12 \\
\hline Energy Usage (kBtu) & 102001.31 & 89019.32 & 7795.13 & 62179.22 & 81803.78 & 88786.49 & 101277.90 & 98015.49 & 78192.00 & 62777.24 & 78956.97 & 125382.57 \\
\hline \multicolumn{13}{|c|}{ Electric Energy Usage (Estimated) } \\
\hline Month & 1 & 2 & 3 & 4. & (Estimated) & (Estimated) & (Estimated) & 8 & 9 & 10 & 11 & 12 \\
\hline Energy Usage (kBtu) & 102001.31 & 89019.32 & 77795.13 & 62179.22 & 79146.27 & 96192.50 & 103900.93 & 98015.49 & 78192.00 & 62777.24 & 78956.97 & 125382.57 \\
\hline Percentage Error & $0.00 \%$ & $0.00 \%$ & $0 . \infty$ & 0.00 & $3.25 \%$ & -8.349 & -2.598 & 0.00 & 0.00 & 0.00 & 0.00 & 0.00 \\
\hline
\end{tabular}

Figure 7. Building 1 actual data also highlighting three estimated months.

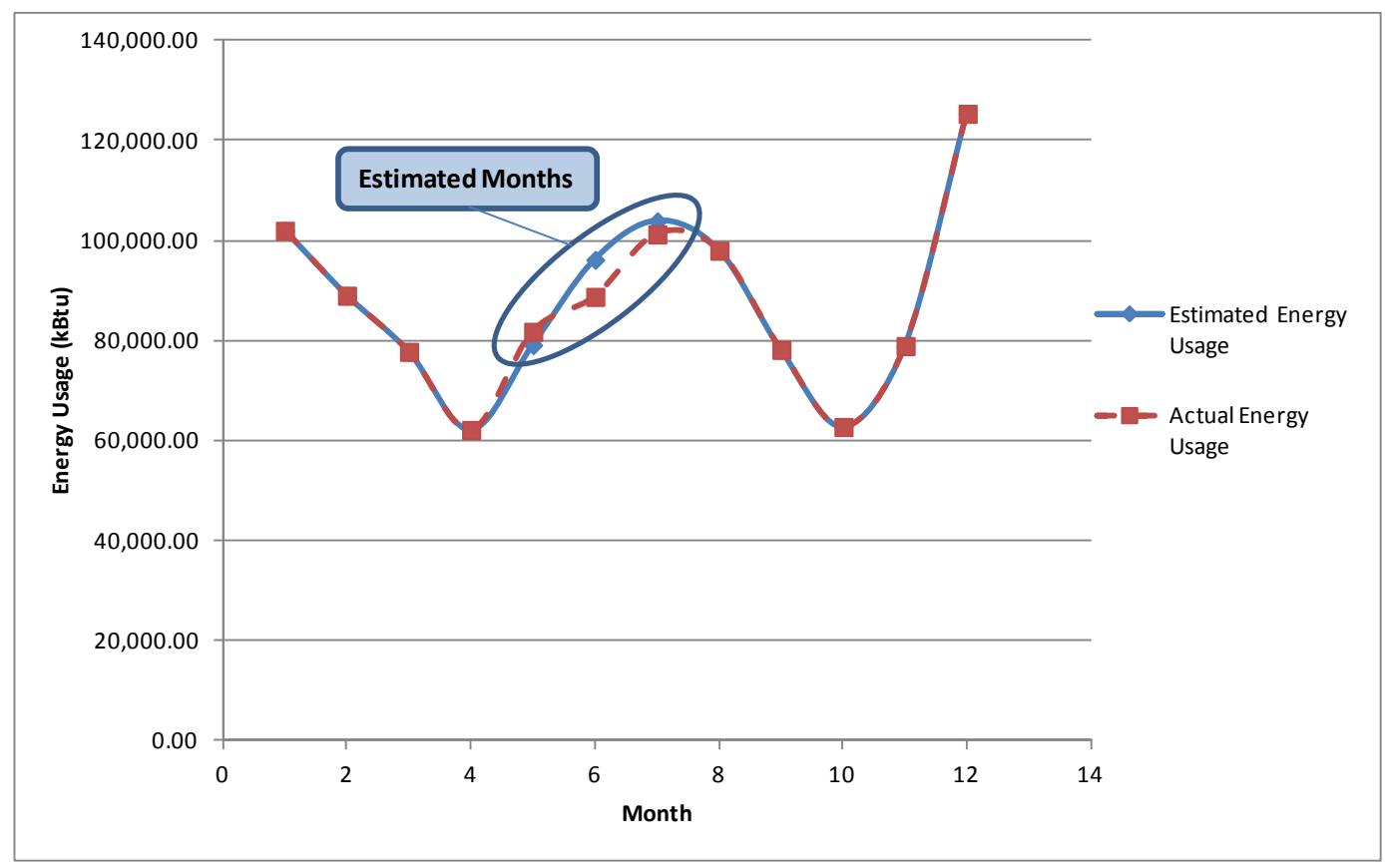

\subsubsection{Estimating missing data-proportional method}

The proportional method was attempted in order to rectify the problems of encountered with the LINSET function. This method utilized a previous or subsequent year of complete data to calculate the missing values for a given building and a given year using a simple proportion relating the annual energy usage to that of the missing months, as shown in Equation 1. It was believed that the proportion could inherently factor annual climate variations and building modifications when calculating the missing data. However, as can be seen in Table 14, the percentage error between an actual set of data and estimated values is quite high. This high error was replicated for other buildings as well (Table 15).

$$
\frac{a_{n}}{Y_{1}-\sum a_{i}}=\frac{b_{n}}{Y_{2}-\sum b_{i}}
$$


where

$$
\left\{\begin{array}{c}
Y_{1}=\text { annual energy consumption for year with complete data } \\
a_{n}=\text { energy consumption for month in } Y_{1} \text { corresponding to } b_{n} \\
b_{n}=\text { energy consumption for missing month in } Y_{2} \\
Y_{2}=\text { annual energy consumption for year with missing months } \\
\sum a_{i}=\text { energy consumption for months in } Y_{1} \text { corresponding to missing months in } Y_{2} \\
\sum b_{i}=\text { Energy consumption for missing months in } Y_{2}
\end{array}\right.
$$

Table 14. Actual vs. estimated energy usage

(3 months, proportional method, Building 1).

\begin{tabular}{|c|c|c|c|c|c|c|c|c|c|c|c|c|}
\hline \multicolumn{13}{|c|}{ Electric Energy Usage 2012} \\
\hline Month & 1 & 2 & 3 & 4 & 5 (Actual) & 6 (Actual) & 7 (Actual) & 8 & 9 & 10 & 11 & 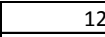 \\
\hline Energy Usage (kBtu) & 109571.97 & 89653.98 & \begin{tabular}{|l|l|l|}
83542.88 & 6 \\
\end{tabular} & 64351.29 & 67280.21 & 68145.15 & 81072.08 & 91705.58 & 81445.27 & 74600.92 & 114377.15 & 84398.00 \\
\hline \multicolumn{13}{|c|}{ Electric Energy Usage 2010 (Actual) } \\
\hline Month & 1 & 2 & 3 & 4 & 5 (Actual) & 5 (Actual) & 5 (Actual) & 8 & 9 & 10 & 11 & 12 \\
\hline Energy Usage (kBtu) & 102001.31 & 89019.32 & \begin{tabular}{|l|l}
77795.13 & 6 \\
\end{tabular} & 62179.22 & 81803.78 & 88786.49 & 101277.90 & 98015.49 & 78192.00 & 62777.24 & 78956.97 & 125382.57 \\
\hline \multicolumn{13}{|c|}{ Electric Energy Usage 2010 (Estimated) } \\
\hline Month & 1 & 2 & 3 & 4 & \begin{tabular}{|l|}
5 (Estimated) \\
\end{tabular} & 6 (Estimated) & \begin{tabular}{|l|}
7 (Estimated) \\
\end{tabular} & 8 & 9 & 10 & 11 & 12 \\
\hline Energy Usage (kBtu) & 102001.31 & 89019.32 & \begin{tabular}{|l|l}
77795.13 & 6 \\
\end{tabular} & 62179.22 & 65641.72 & 66485.60 & 79097.72 & \begin{tabular}{|l|}
98015.49 \\
\end{tabular} & 78192.00 & \begin{tabular}{|l|}
62777.24 \\
\end{tabular} & 78956.97 & 125382.57 \\
\hline Percentage Error & $0.00 \%$ & $0.00 \%$ & $0.00 \%$ & $0.00 \%$ & $19.76 \%$ & $25.12 \%$ & $21.90 \%$ & $0.00 \%$ & $0.00 \%$ & $0.00 \%$ & $0.00 \%$ & 0.00 \\
\hline
\end{tabular}

Table 15. Actual vs. estimated energy usage

(3 months, proportional method, Building 2).

\begin{tabular}{|c|c|c|c|c|c|c|c|c|c|c|c|c|}
\hline \multicolumn{13}{|c|}{ Electric Energy Usage 2012} \\
\hline Month & 1 & 2 & 3 & 4 & 5 (Actual) & 6 (Actual) & 7 (Actual) & 8 & 9 & 10 & 11 & 12 \\
\hline Energy Usage (kBtu) & 44010.46 & 39092.79 & 12797.00 & 41609.16 & 35204.57 & 39067.97 & 35766.48 & 32191.00 & 34759.93 & 33259.21 & 34181.13 & 23808.71 \\
\hline \multicolumn{13}{|c|}{ Electric Energy Usage 2013 (Actual) } \\
\hline Month & 1 & 2 & 3 & 4 & 5 (Actual) & 5 (Actual) & 5 (Actual) & 8 & 9 & 10 & 11 & 12 \\
\hline Energy Usage (kBtu) & 28437.03 & 20355.47 & 27146.35 & 20493.77 & 22661.74 & 28152.49 & 32605.58 & 32349.26 & 28112.19 & 22094.06 & 24649.96 & 26341.74 \\
\hline \multicolumn{13}{|c|}{\begin{tabular}{|l} 
Electric Energy Usage 2013 (Estimated) \\
(s)
\end{tabular}} \\
\hline Month & 1 & 2 & 3 & 4 & 5 (Estimated) & 6 (Estimated) & 7 (Estimated) & 8 & 9 & 10 & 11 & 12 \\
\hline Energy Usage (kBtu) & 28437.03 & 20355.47 & 27146.35 & 20493.77 & 27379.39 & 30384.04 & 27816.40 & 32349.26 & 28112.19 & 22094.06 & 24649.96 & 26341.74 \\
\hline Percentage Error & $0.00 \%$ & $0.00 \%$ & $0.00 \%$ & $0.00 \%$ & $-20.82 \%$ & $-7.93 \%$ & $14.69 \%$ & $0.00 \%$ & $0.00 \%$ & $0.00 \%$ & $0.00 \%$ & $0.00 \%$ \\
\hline
\end{tabular}

Given the relatively high error rate for both methods, it was determined that estimating the missing data would increase the uncertainty of the final results. Therefore, neither method was implemented. Only the buildings with at least one complete, uninterrupted year of data were considered. Although this reduced the size of the available data set, it ensured that the data were representative of the actual conditions.

\subsection{Results}

The final set of 71 buildings provided a means to investigate various energy consumption statistics at Army installations. The results of these investigations were then used to establish the benchmarking criteria. To understand the relationships between the various investigated parameters, the following charts were generated for each building type: 
- EUI vs. Construction Year

- EUI vs. Floor Area ( $\left.\mathrm{ft}^{2}\right)$

- Annual Energy Consumption vs. Floor Area ( $\left.\mathrm{ft}^{2}\right)$

- EUI (Electric, Gas, and Combined) vs. Climate Zone

- Annual Energy Consumption vs. Climate Zone

Additionally, mean and median EUIs were calculated for the following criteria:

- Construction Year

- Construction Type

- Floor Area ( $\left.\mathrm{ft}^{2}\right)$

The results of parameter comparisons and analyses are discussed in the sections that follow.

\subsubsection{Parameter relationships: construction year and construction type}

Figure 8, Figure 9, and Figure 10 compare barracks, dining facility, and vehicle maintenance building EUIs, respectively, to the year the building was constructed. From these charts, it can be seen that most buildings were constructed in the 1960s or after the 1980s. Barracks constructed after 1960 but before 1980 tend to have higher EUIs than other buildings, while DFACs have relatively uniform EUIs over all construction years. Conversely, vehicle maintenance buildings have become more energy inefficient in recent years, with the lowest EUIs seen among buildings built between the 1960s and the 1980s.

Some of these trends can be explained by the location of construction. For example, most vehicle maintenance buildings built in the 1960s were located in Climate Zone 5B, which experiences relatively temperate weather patterns. On the contrary, barracks built in the 1960s were located in Climate Zone 4A, which experiences greater weather extremes. Nevertheless, even within climate zones, there appears to be a decrease in EUI for barracks built before 1960 and after 1980, while EUIs seem to increase for vehicle maintenance buildings built in the same timeframe.

When comparing construction years to construction types (Figure 11) for these buildings, it is seen that block, brick veneer, and "other" construction techniques were the most common construction methods until recently. This may partially explain the relationship between building EUI 
and the year of construction. As building methods have progressed over the years, buildings have become more energy efficient with tighter envelopes and reduced energy consumption.

More variations between construction types and construction years can been seen in Table 16 and Table 17, which compare mean and median EUIs, respectively, to these parameters for a given building type.

Figure 8. EUI vs. construction year (barracks).

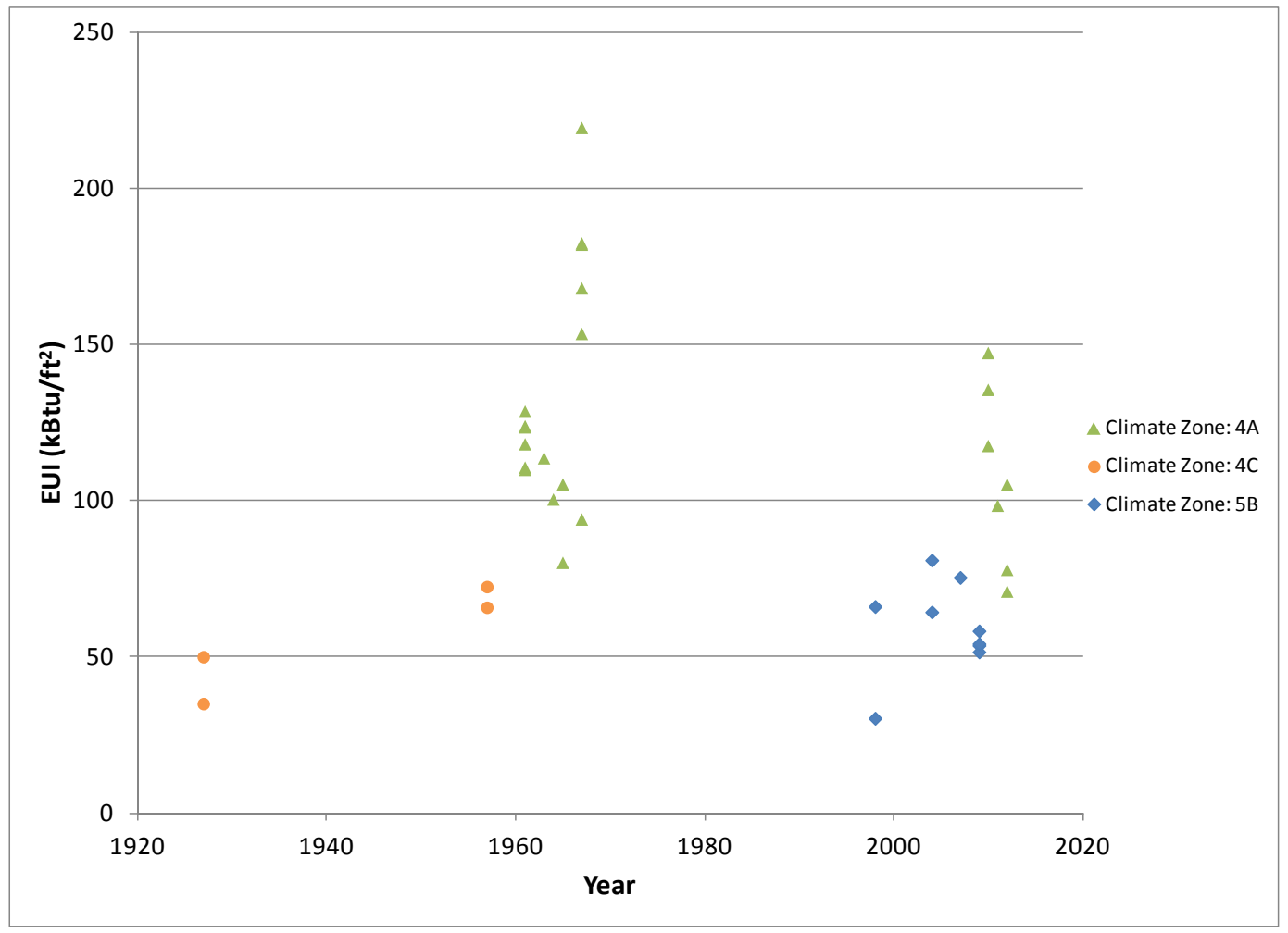


Figure 9. EUI vs. construction year (DFACs).

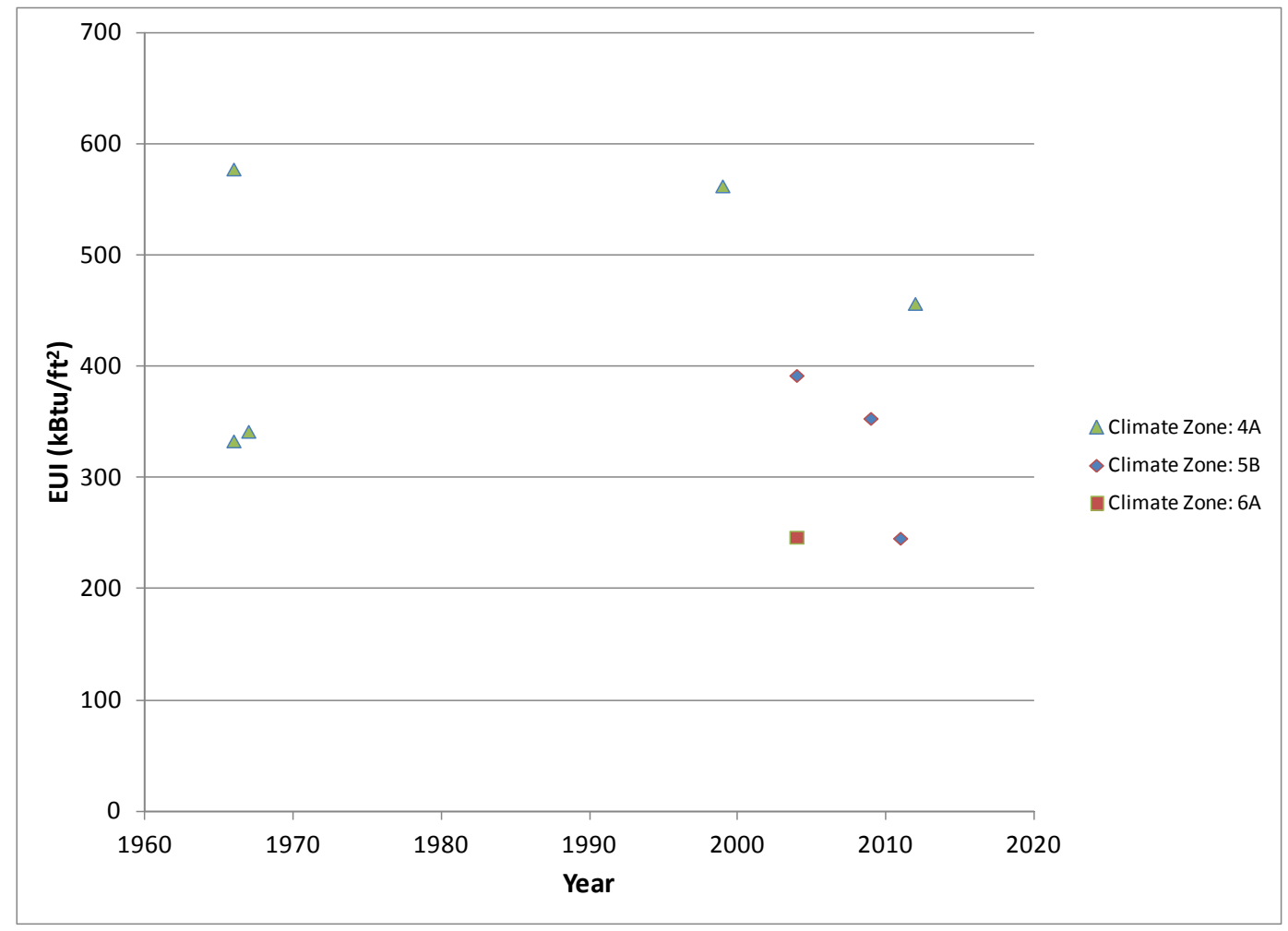

Figure 10. EUI vs. construction year (vehicle maintenance).

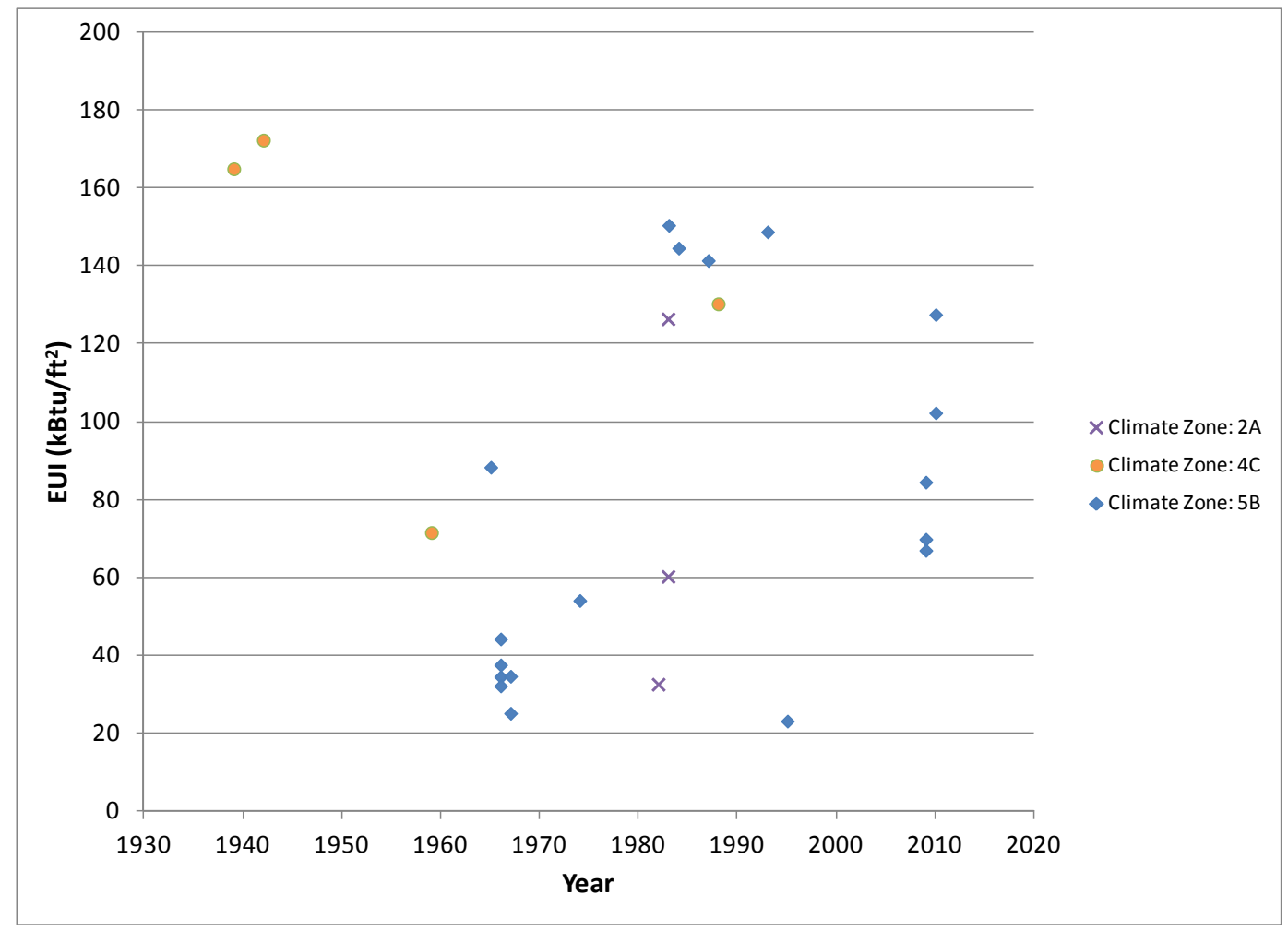


Figure 11. Construction year vs. construction type.

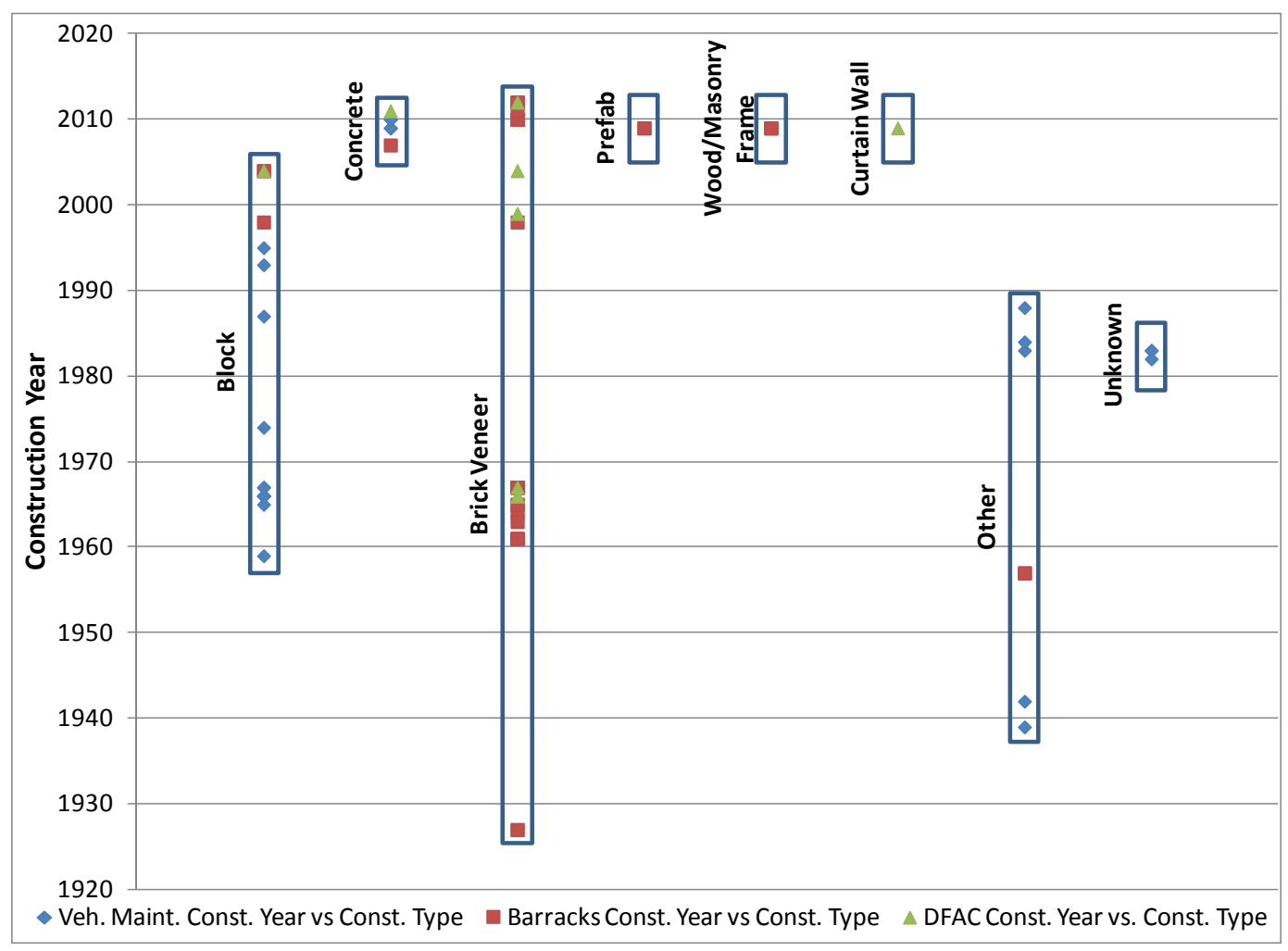

Table 16. Construction year and construction type mean EUls.

\begin{tabular}{|c|c|c|c|}
\hline Construction Year & Barracks & DFACs & Veh. Maint. \\
\hline Before 1950 & 42.30 & $\mathrm{~N} / \mathrm{A}$ & 168.80 \\
\hline $1950-1960$ & 68.94 & $\mathrm{~N} / \mathrm{A}$ & 71.68 \\
\hline $1960-1970$ & 131.96 & 417.07 & 42.50 \\
\hline $1970-1980$ & $\mathrm{~N} / \mathrm{A}$ & $\mathrm{N} / \mathrm{A}$ & 54.23 \\
\hline $1980-1990$ & $\mathrm{~N} / \mathrm{A}$ & $\mathrm{N} / \mathrm{A}$ & 112.37 \\
\hline $1990-2000$ & 48.23 & 561.99 & 86.09 \\
\hline $2000-2010$ & 59.06 & 330.23 & 73.90 \\
\hline After 2010 & 107.44 & 350.75 & 115.04 \\
\hline Construction Type & Barracks & DFACs & Veh. Maint. \\
\hline Block & 58.55 & 391.56 & 61.43 \\
\hline Brick Veneer & 115.93 & 419.28 & $\mathrm{~N} / \mathrm{A}$ \\
\hline Combo: Wood/Masonry Frame & 53.14 & $\mathrm{~N} / \mathrm{A}$ & $\mathrm{N} / \mathrm{A}$ \\
\hline Concrete & 75.41 & 245.20 & 90.35 \\
\hline Curtain Wall & $\mathrm{N} / \mathrm{A}$ & 352.95 & N/A \\
\hline Metal & $\mathrm{N} / \mathrm{A}$ & N/A & N/A \\
\hline Other & 68.94 & $\mathrm{~N} / \mathrm{A}$ & 152.68 \\
\hline Prefab & 46.19 & N/A & $\mathrm{N} / \mathrm{A}$ \\
\hline
\end{tabular}


Table 17. Construction year and construction type median EUls.

\begin{tabular}{|c|c|c|c|}
\hline Construction Year & Barracks & DFACs & Veh. Maint. \\
\hline Before 1950 & 42.30 & $\mathrm{~N} / \mathrm{A}$ & 168.80 \\
\hline $1950-1960$ & 68.94 & $\mathrm{~N} / \mathrm{A}$ & 71.68 \\
\hline $1960-1970$ & 120.69 & 341.34 & 34.78 \\
\hline $1970-1980$ & $\mathrm{~N} / \mathrm{A}$ & $\mathrm{N} / \mathrm{A}$ & 54.23 \\
\hline $1980-1990$ & $\mathrm{~N} / \mathrm{A}$ & $\mathrm{N} / \mathrm{A}$ & 130.44 \\
\hline $1990-2000$ & 48.23 & 561.99 & 86.09 \\
\hline $2000-2010$ & 56.24 & 352.95 & 69.97 \\
\hline After 2010 & 105.11 & 350.75 & 115.04 \\
\hline Construction Type & Barracks & DFACs & Veh. Maint. \\
\hline Block & 64.35 & 391.56 & 41.02 \\
\hline Brick Veneer & 112.01 & 398.82 & $\mathrm{~N} / \mathrm{A}$ \\
\hline Combo: Wood/Masonry Frame & 53.64 & $\mathrm{~N} / \mathrm{A}$ & $\mathrm{N} / \mathrm{A}$ \\
\hline Concrete & 75.41 & 245.20 & 84.63 \\
\hline Curtain Wall & $\mathrm{N} / \mathrm{A}$ & 352.95 & $\mathrm{~N} / \mathrm{A}$ \\
\hline Metal & $\mathrm{N} / \mathrm{A}$ & $\mathrm{N} / \mathrm{A}$ & $\mathrm{N} / \mathrm{A}$ \\
\hline Other & 68.94 & $\mathrm{~N} / \mathrm{A}$ & 150.61 \\
\hline Prefab & 46.19 & $\mathrm{~N} / \mathrm{A}$ & $\mathrm{N} / \mathrm{A}$ \\
\hline
\end{tabular}

\subsubsection{Parameter relationships: floor area}

As seen in Figure 12, Figure 14, and Figure 16, the relationships between EUI and floor area generally meet expectations as do the relationships between total energy consumption and floor area (Figure 13, Figure 15, and Figure 17). Energy consumption tends to increase with square footage, while EUIs remain relatively constant as square footage increase. Two discrepancies from these expectations must be noted, however.

Among barracks, buildings in Climate Zone 4A seem to have different EUIs for the same floor area. EUIs range from 70.85 to $218.25 \mathrm{kBTU} / \mathrm{ft}^{2}$. Similarly, EUIs for vehicle maintenance buildings in Climate Zone 5b do not remain constant as floor area changes. For this building type at this location, EUI tends to increase with square footage. This implies that other factors dictate the energy consumption of these buildings more than square footage alone. 
Figure 12. EUI vs. floor area (barracks).

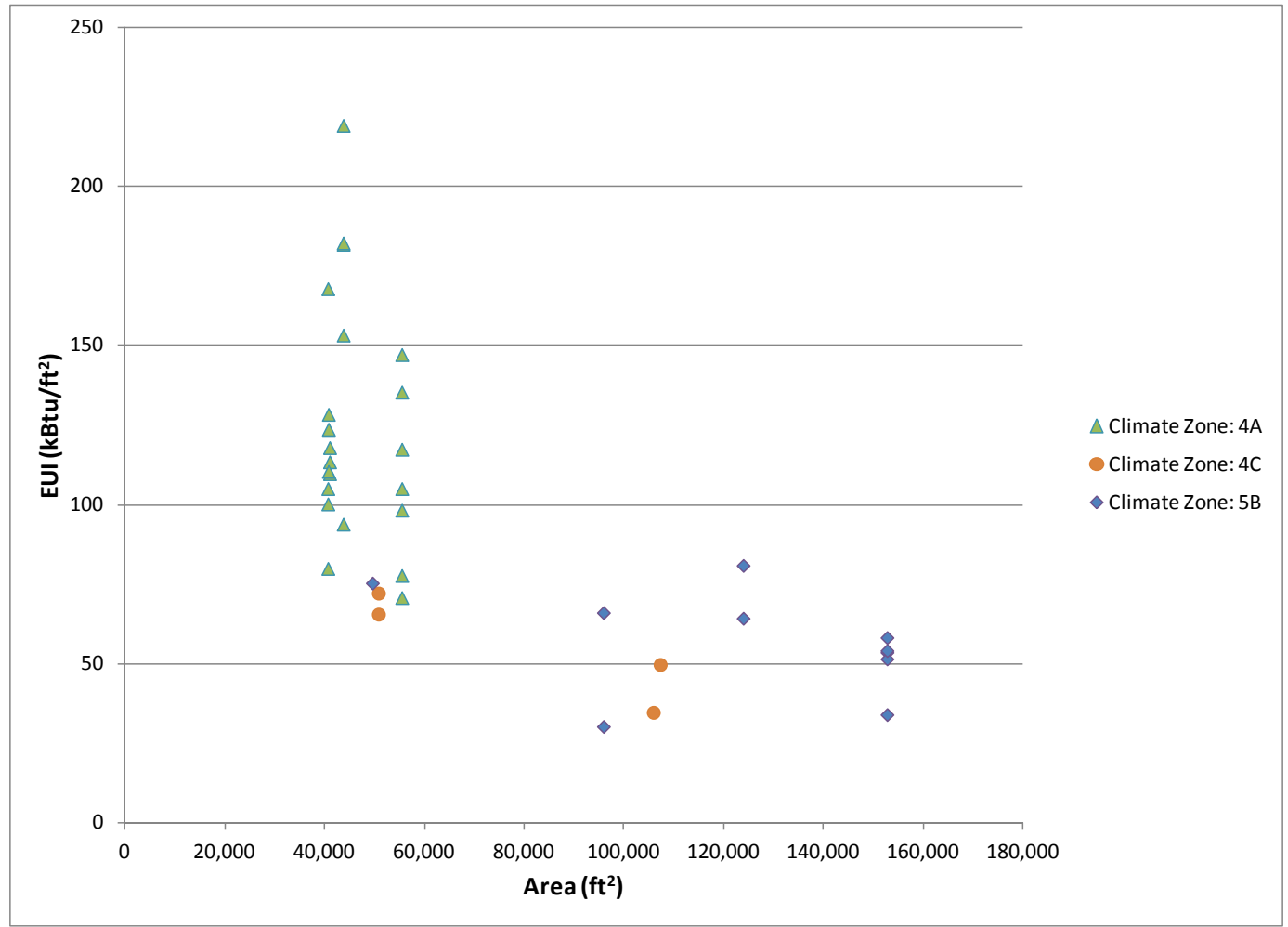

Figure 13. Annual energy consumption vs. floor area (barracks).

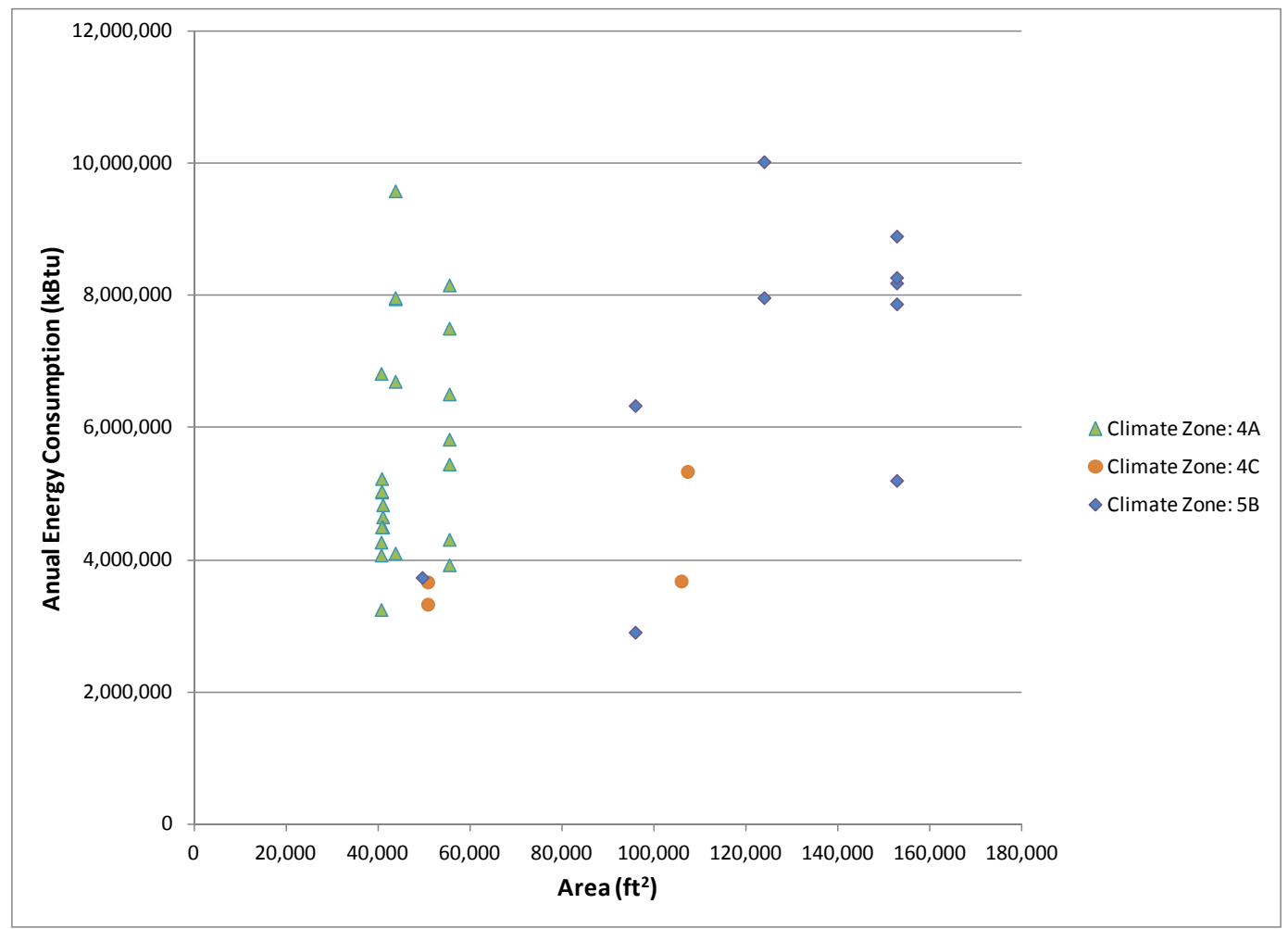


Figure 14. EUI vs. floor area (DFACs).

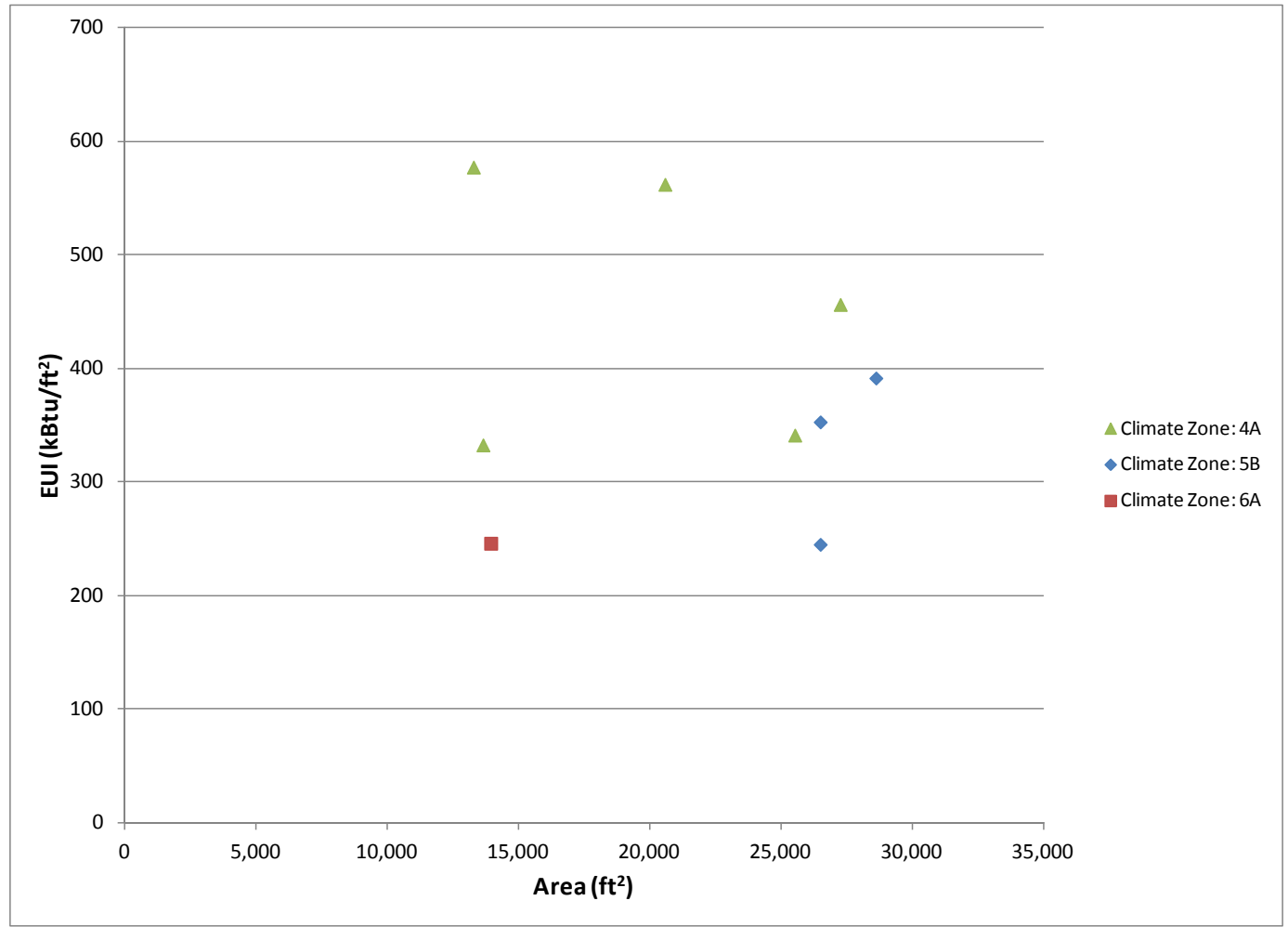

Figure 15. Annual energy consumption vs. floor area (DFACs).

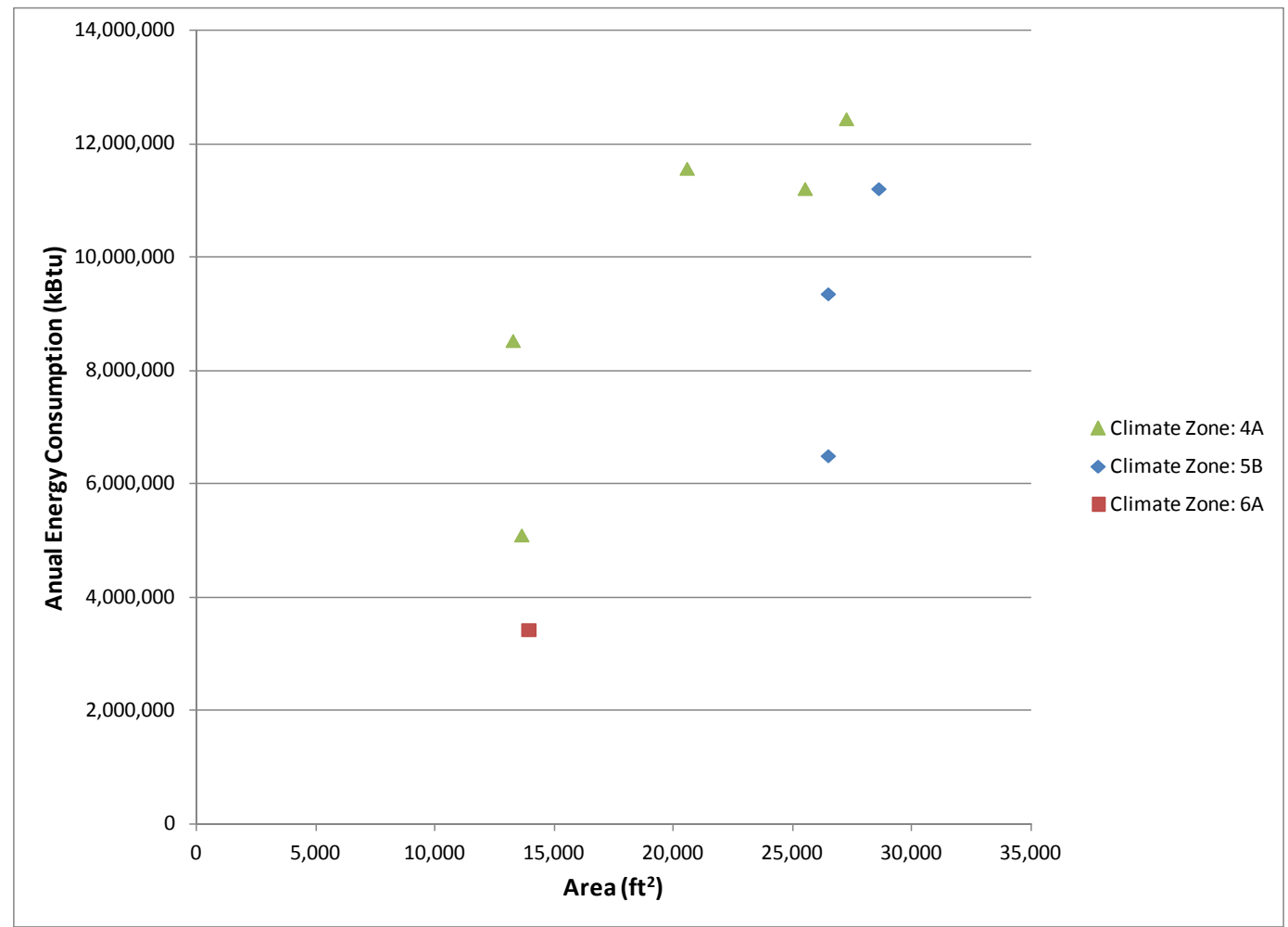


Figure 16. EUI vs. floor area (vehicle maintenance).

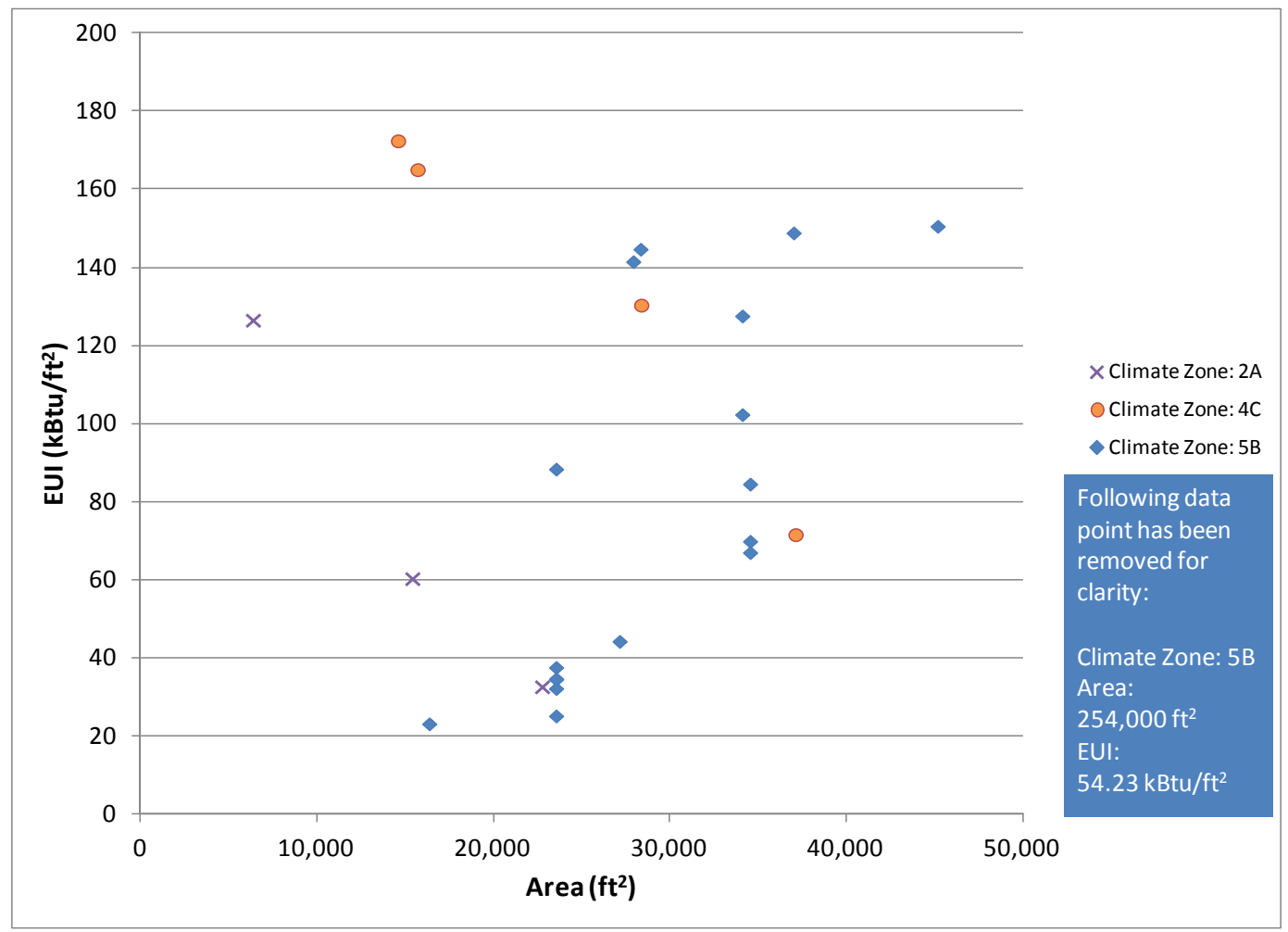

Figure 17. Annual energy consumption vs. floor area (vehicle maintenance).

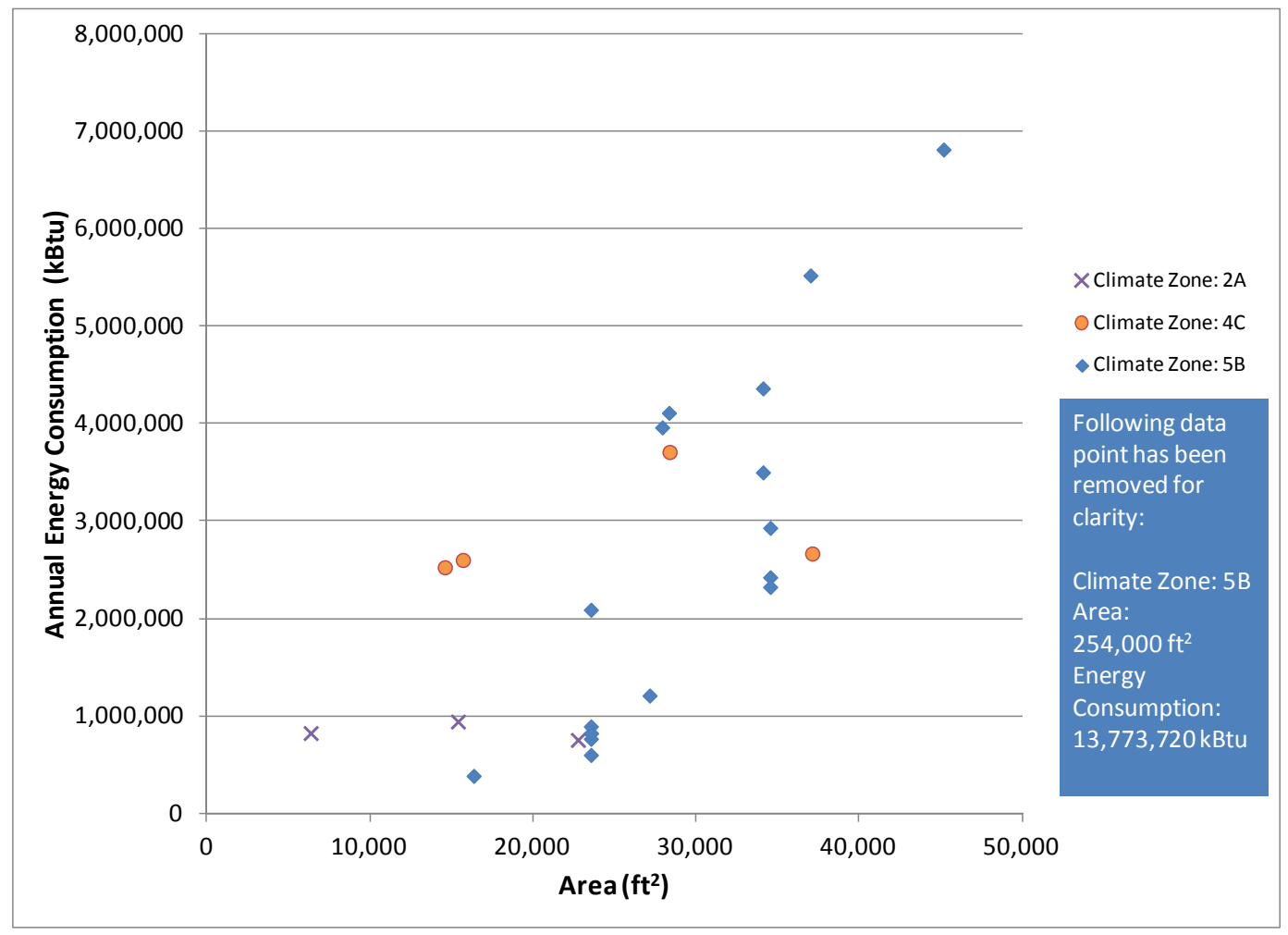




\subsubsection{Parameter relationships: location}

As discussed in the previous sections, building location appears to influence energy usage more than the other investigated parameters. This is as expected given the temperature and weather variations between climate zones. Figure 18, Figure 21, and Figure 24 highlight the effects of these location-based differences. Figure 19, Figure 22, and Figure 25 further segment the data into gas and electric EUIs for each location and building type, while Figure 20, Figure 23, and Figure 26 plot electric and gas energy consumption at the various climate zones.

From these charts, one can see that Climate Zone 4A tends to have the highest EUIs as well as higher annual energy consumption levels. Buildings in Zone 4A appear to consume more gas and electricity than similar buildings in other climate zones given similar floor areas. As explained above, this result may be explained by the greater temperature/ weather variations associated with the area.

The segmented data show that buildings tend to use more natural gas than electricity as measured in $\mathrm{kBtu} / \mathrm{ft}^{2}$. This relationship could be explained by various factors but without more data and a more detailed analysis a definite conclusion cannot be drawn. Some possibilities include the need for domestic hot water, greater heating inefficiencies, or the need to reheat chilled air for humidity control. Interestingly, this trend is less relevant for barracks in Climate Zone 5B, where electricity and gas consumption are more uniform in terms of $\mathrm{kBtu} / \mathrm{ft}^{2}$. Although a definite conclusion cannot be determined for this relationship either, the relatively temperate climate may be a factor. 
Figure 18. EUI vs. location (barracks).

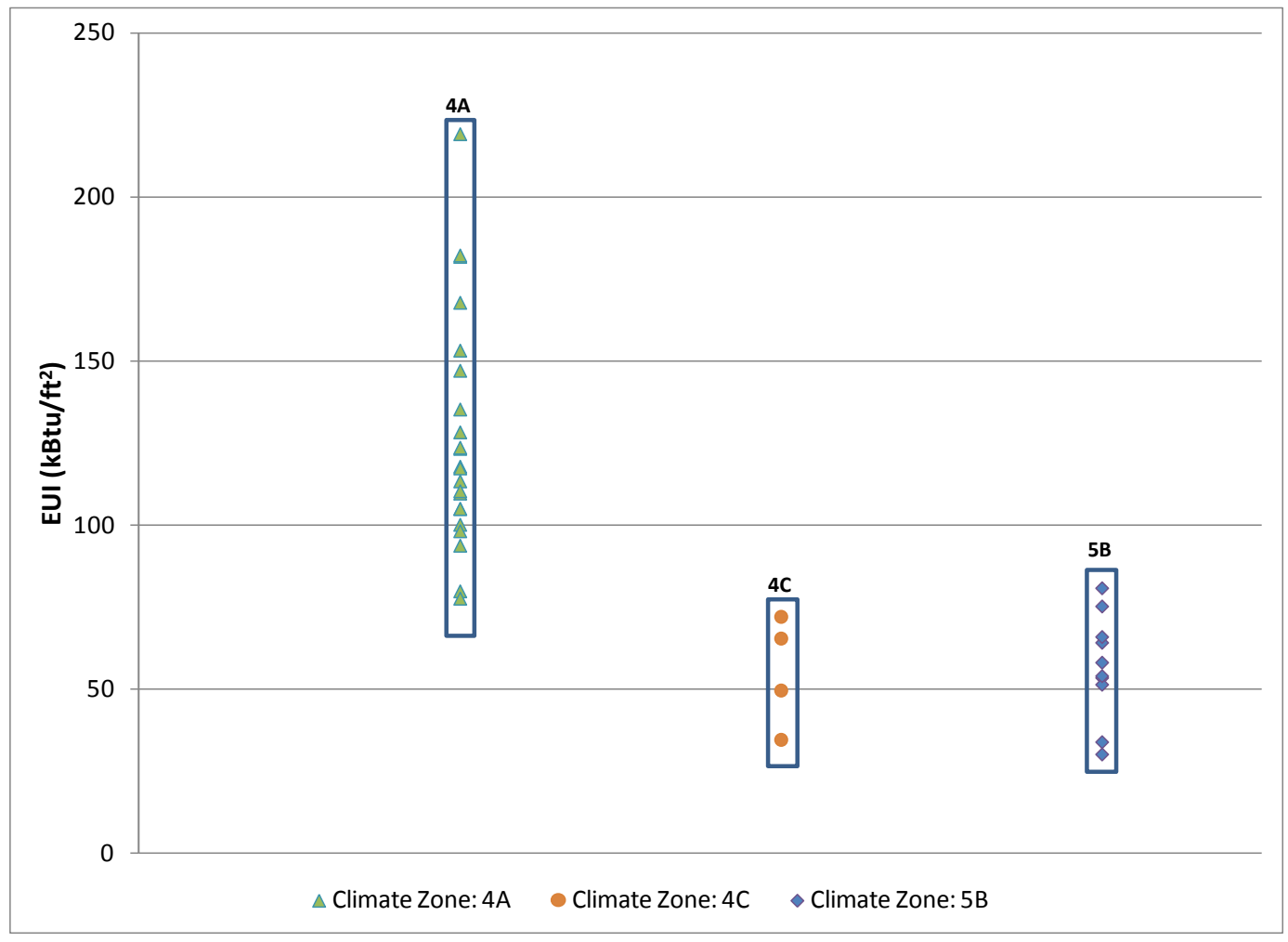

Figure 19. Electric and gas EUI vs. location (barracks).

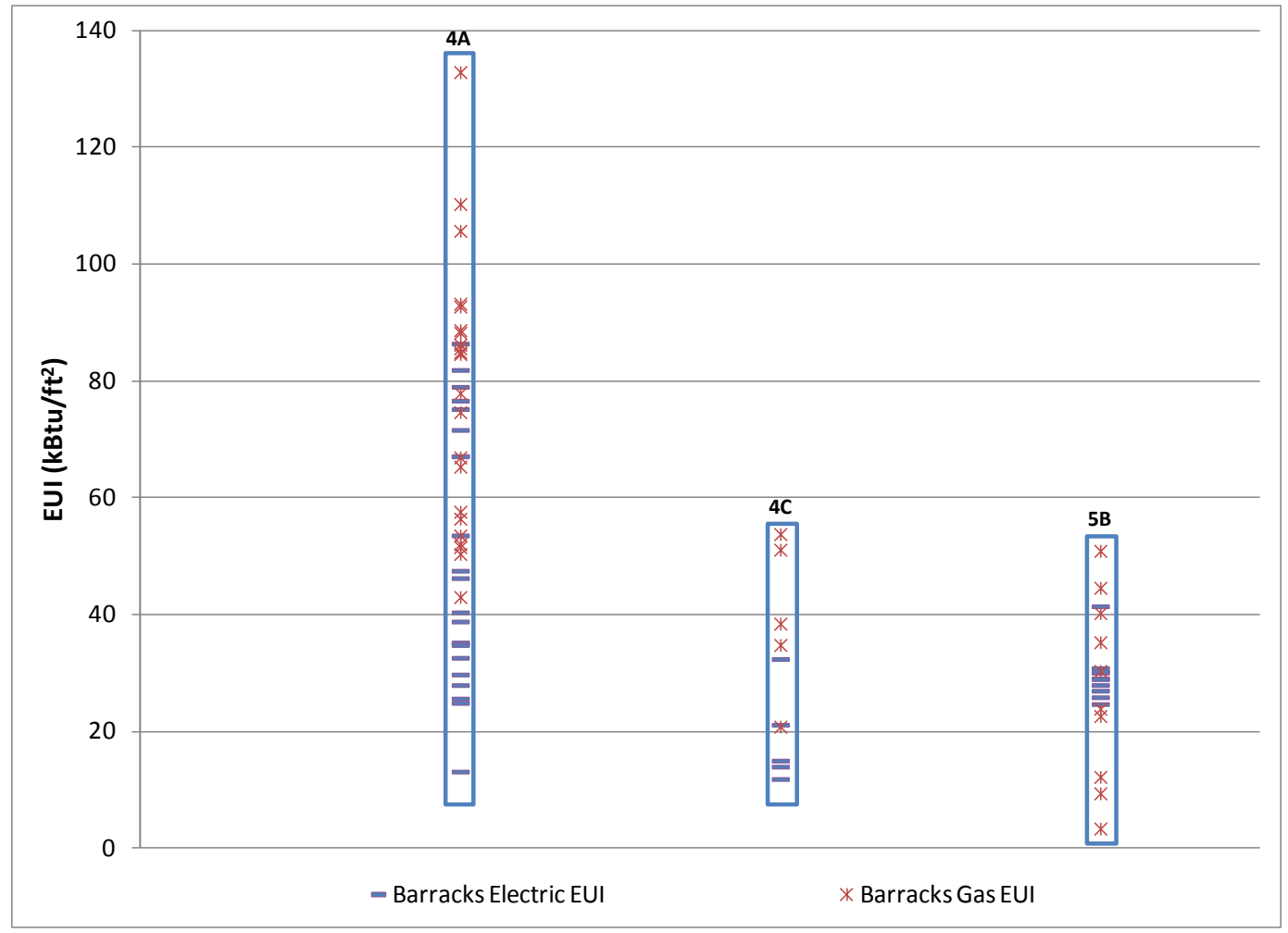


Figure 20. Electric and gas annual energy consumption vs. location (barracks).

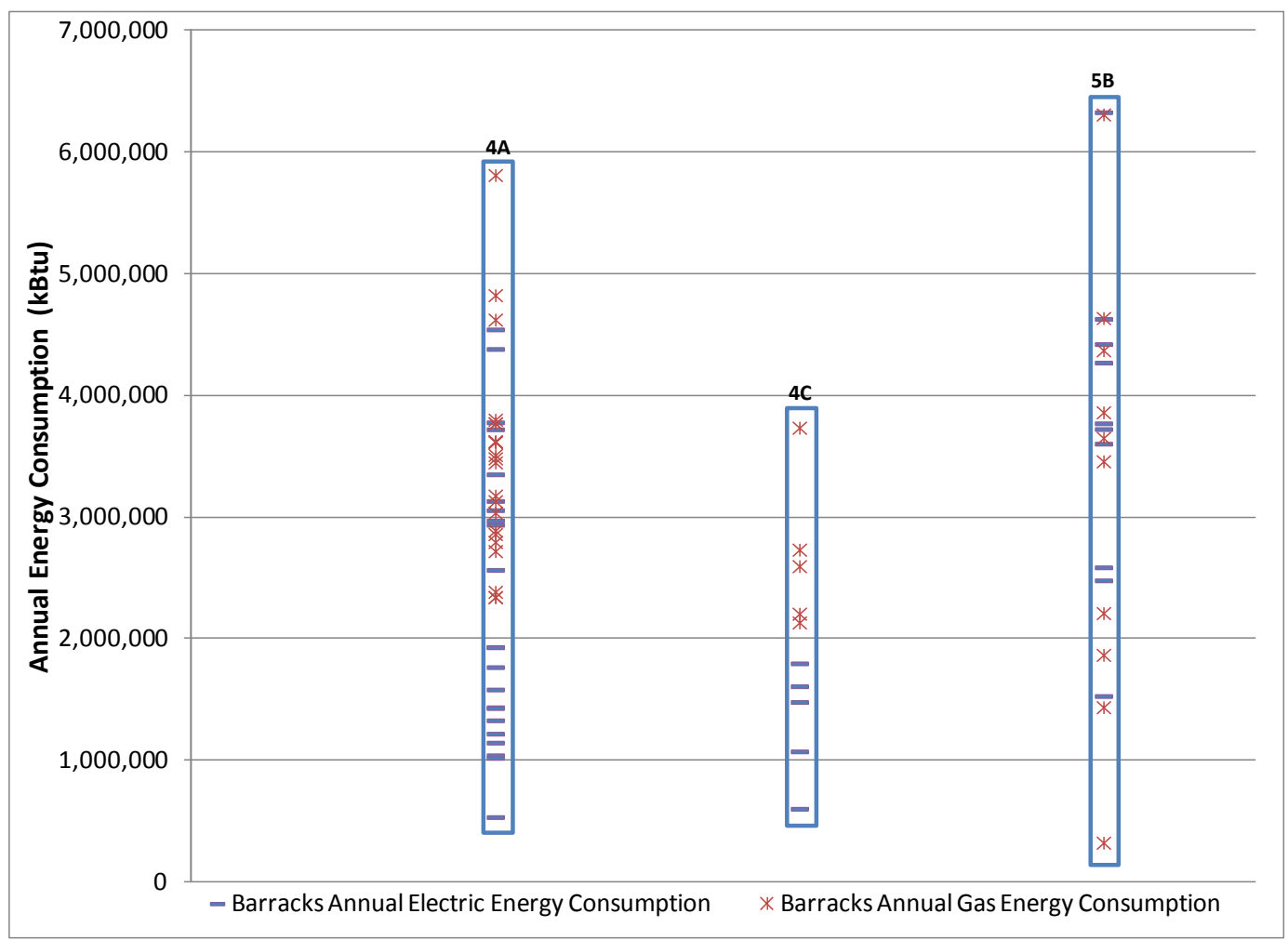

Figure 21. EUI vs. location (DFACs).

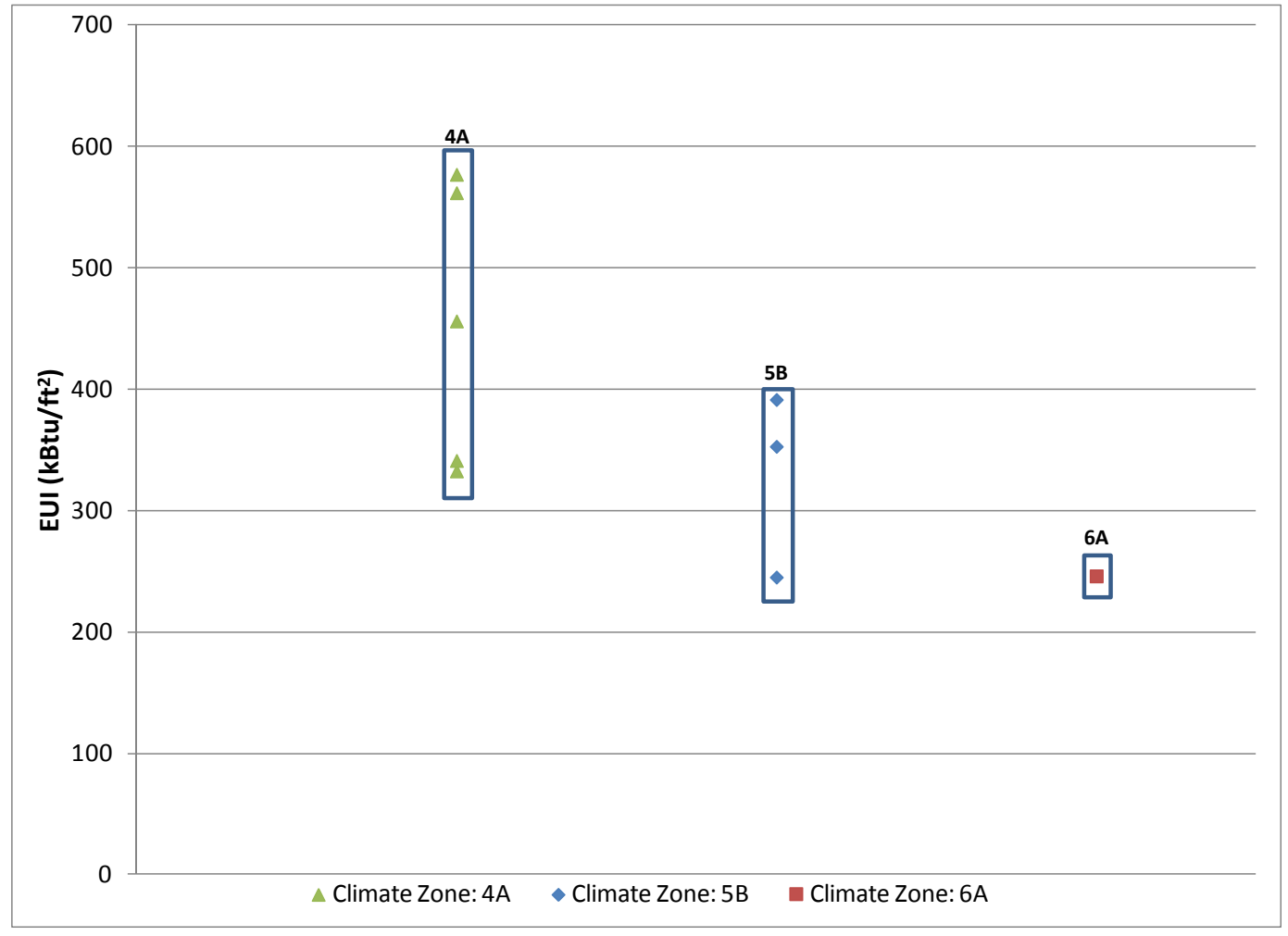


Figure 22. Electric and gas EUI vs. location (DFACs).

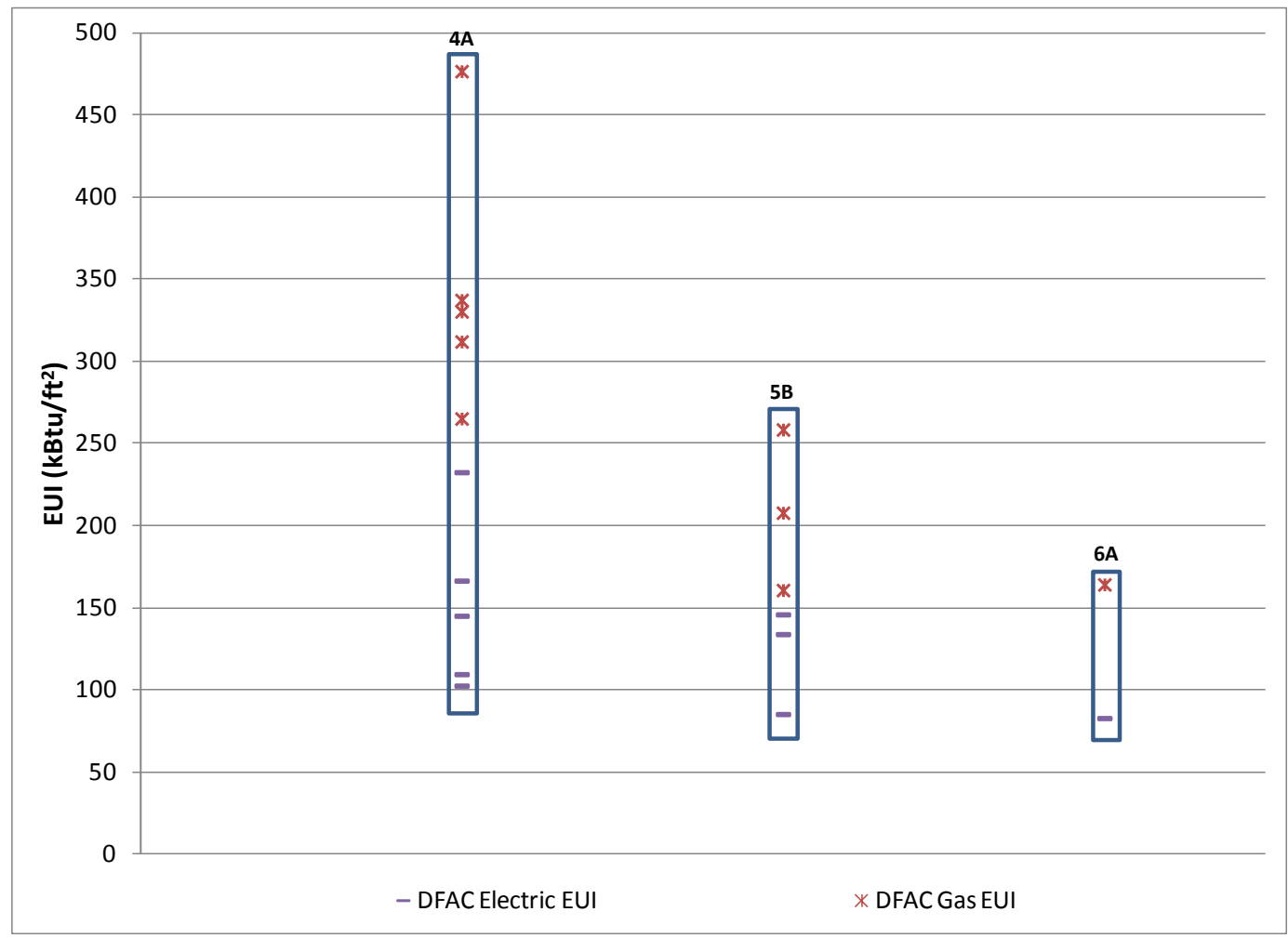

Figure 23. Electric and gas annual energy consumption vs. location (DFACs).

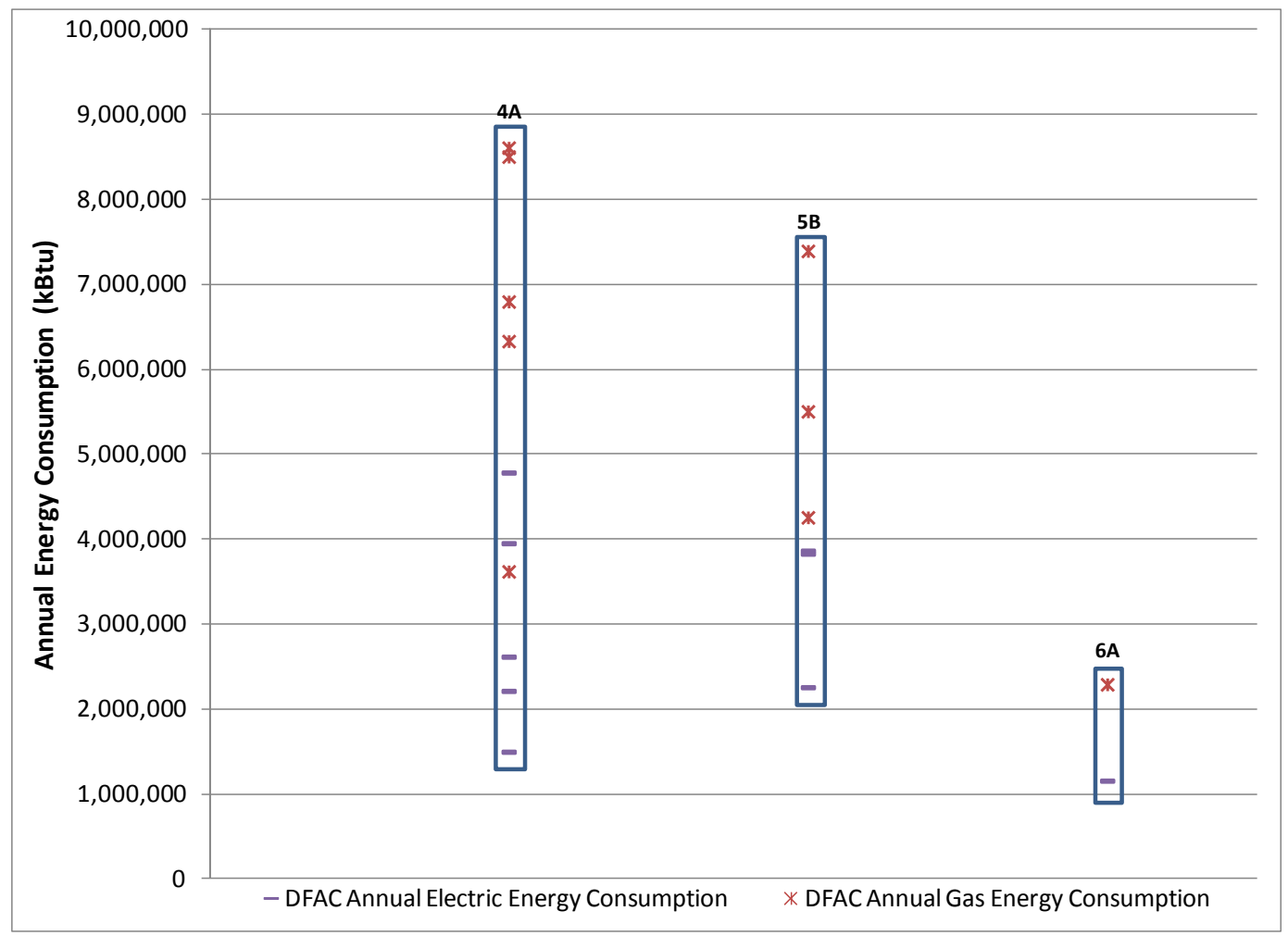


Figure 24. EUI vs. location (vehicle maintenance).

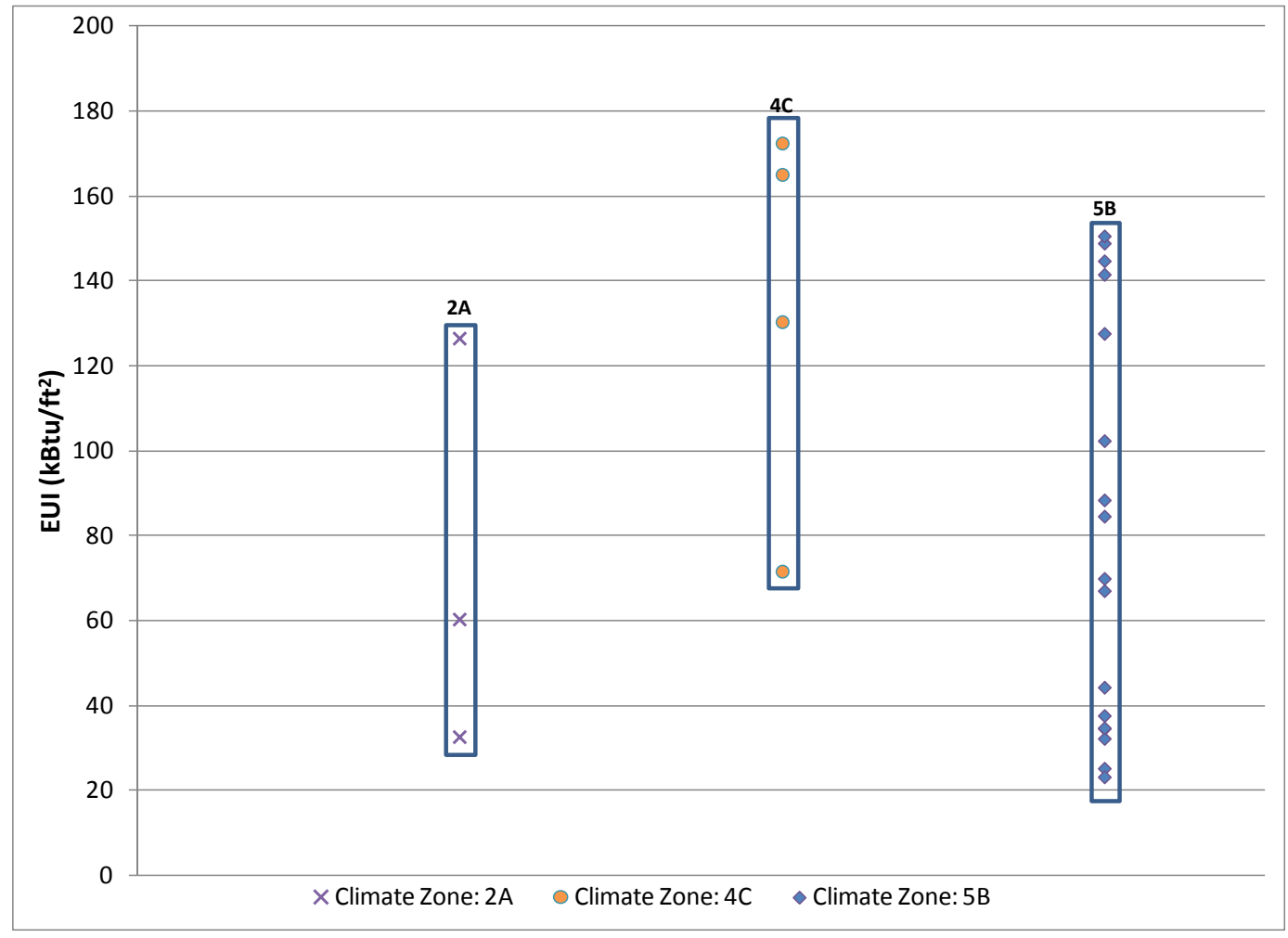

Figure 25. Electric and gas EUI vs. location (vehicle maintenance).

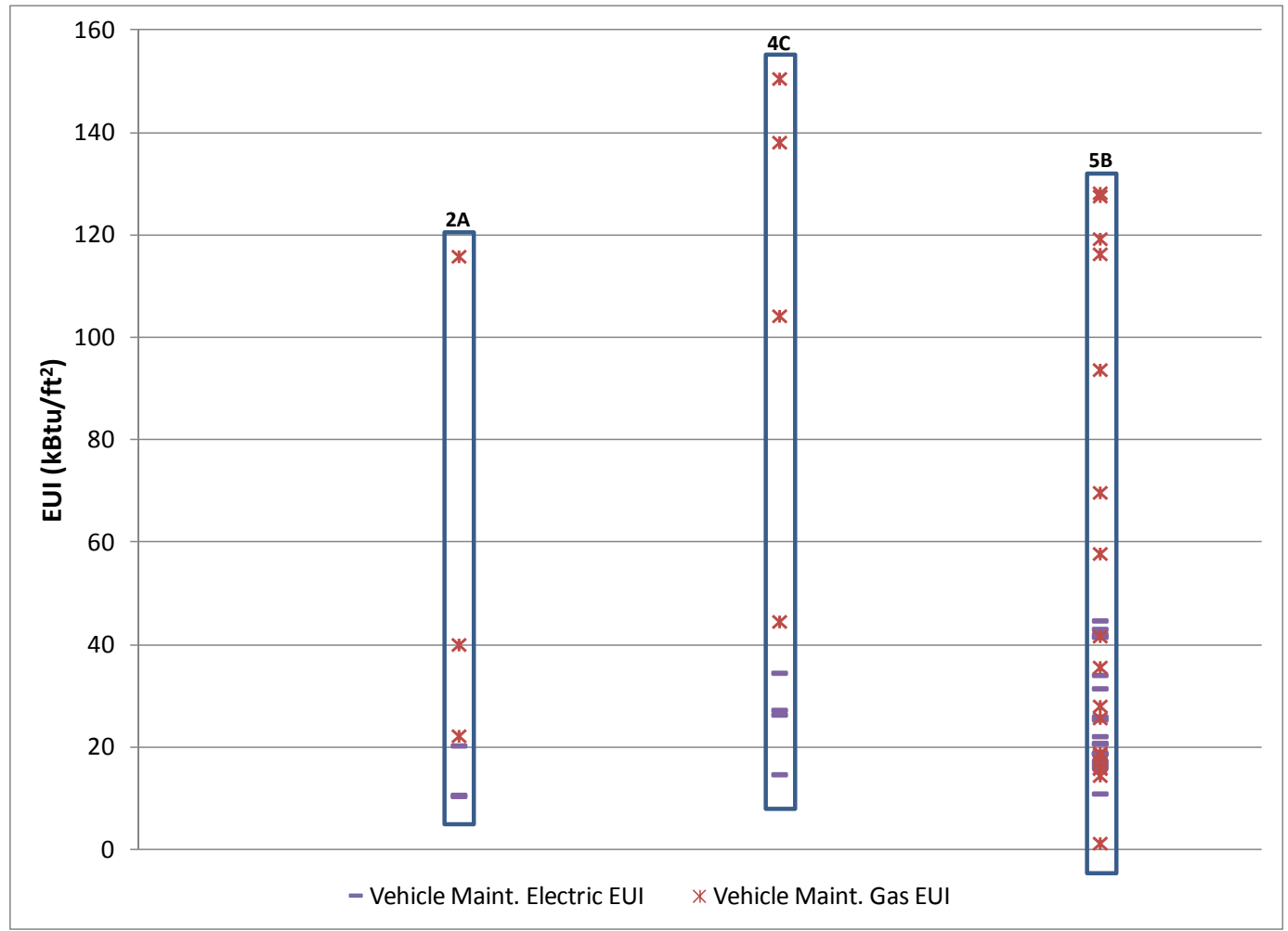


Figure 26. Electric and gas annual energy consumption vs. location (vehicle maintenance).

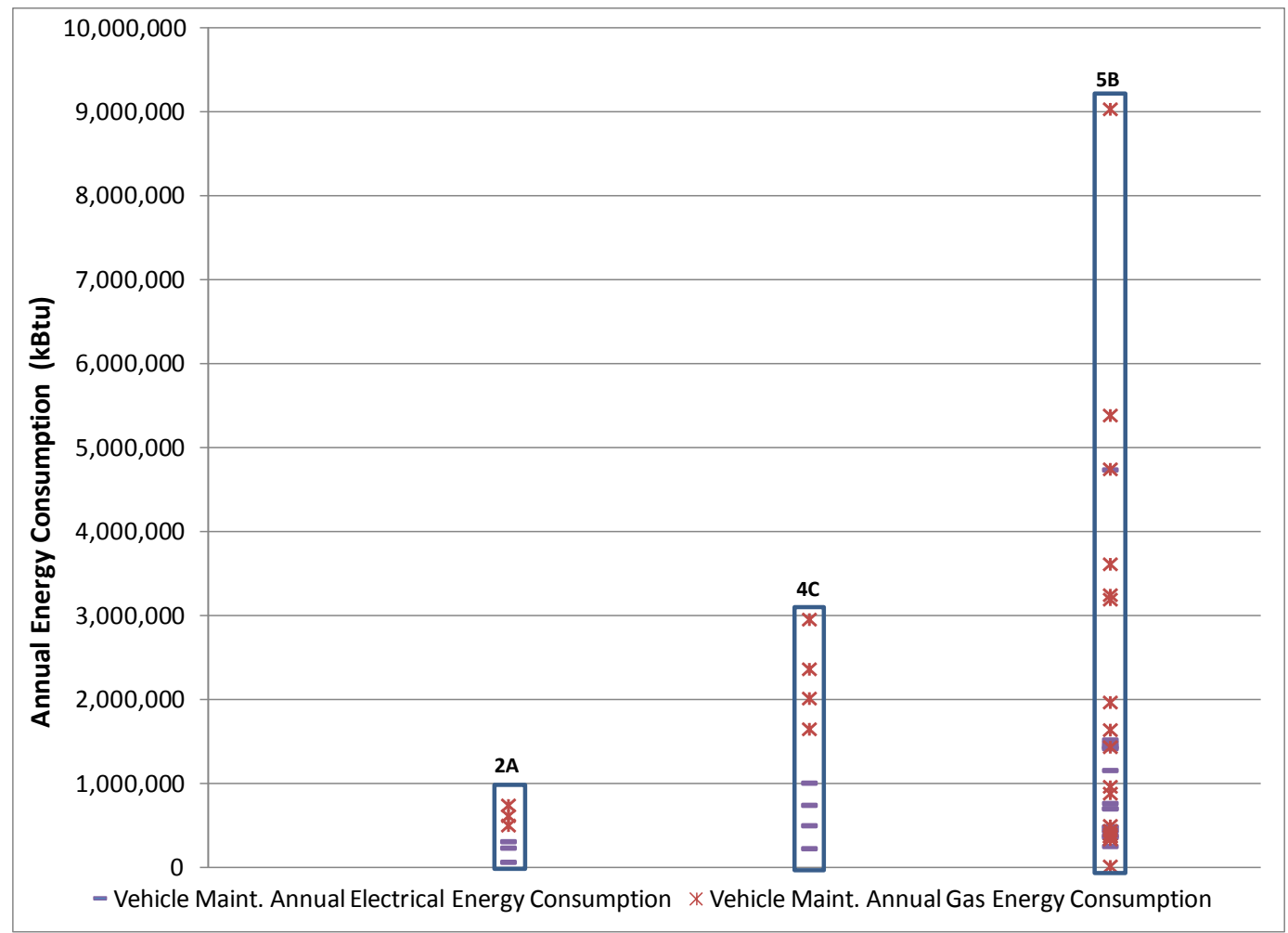

\subsubsection{Parameter relationships between occupancy and usage}

Although this study did not explicitly address the occupancy of each analyzed building, building usage appears to be a primary factor in determining overall energy consumption. In the relationships explored previously, inter-criteria discrepancies can be explained by various means, but intracriteria variations are best explained by differences in building usage and occupancy.

For the data presented in section 4.5.3, one observes a relatively large spread of EUIs for buildings in the same climate zone and of the same building type. Table 18, Table 19, and Table 20 quantify this spread in the data by comparing the median, mean, range, and standard deviation for each climate zone and building type. These tables and the figures above show the relatively large variation in EUIs for buildings in Zone 4A. Both barracks and DFACs in Zone 4A have larger ranges and standard deviations than similar buildings at other locations. Additionally, it is seen in A1 and Table A2 of the Appendix that Zone 4A buildings have similar construction types and years, so those criteria cannot account for the variations in energy consumption. Variations in building occupancy and usage 
may explain these results, but further study would be required to determine the exact relationship.

Table 18. Data spread/variation (barracks).

\begin{tabular}{|c|r|r|r|r|}
\hline EUIs (kBtu/ft ${ }^{2}$ ) & \multicolumn{1}{|c|}{ All } & \multicolumn{1}{c|}{ Climate Zone: 4A } & Climate Zone: 4C & \multicolumn{1}{c|}{ Climate Zone: 5B } \\
\hline Median & 98.32 & 117.45 & 57.72 & 56.24 \\
\hline Mean & 98.78 & 124.50 & 55.62 & 56.90 \\
\hline Range & 188.90 & 148.40 & 37.45 & 50.61 \\
\hline Standard Deviation & 45.03 & 36.68 & 16.78 & 16.11 \\
\hline
\end{tabular}

Table 19. Data spread/variation (DFACs).

\begin{tabular}{|c|c|r|r|r|}
\hline EUls (kBtu/ft ${ }^{2}$ ) & All & Climate Zone: 4A & Climate Zone: 5B & \multicolumn{1}{c|}{ Climate Zone: 6A } \\
\hline Median & 352.95 & 456.30 & 352.95 & 246.19 \\
\hline Mean & 389.49 & 453.90 & 329.90 & 246.19 \\
\hline Range & 331.94 & 244.42 & 146.35 & 0.00 \\
\hline Standard Deviation & 121.28 & 116.44 & 75.85 & $\mathrm{~N} / \mathrm{A}$ \\
\hline
\end{tabular}

Table 20. Data spread/variation (vehicle maintenance).

\begin{tabular}{|c|r|r|r|r|}
\hline EUls (kBtu/ft ${ }^{2}$ ) & \multicolumn{1}{|c|}{ All } & \multicolumn{1}{c|}{ Climate Zone: 4A } & Climate Zone: 5B & \multicolumn{1}{c|}{ Climate Zone: 6 A } \\
\hline Median & 71.68 & 60.24 & 68.53 & 147.78 \\
\hline Mean & 86.86 & 73.06 & 78.48 & 134.93 \\
\hline Range & 149.24 & 93.84 & 127.36 & 100.81 \\
\hline Standard Deviation & 49.67 & 48.22 & 46.73 & 45.98 \\
\hline
\end{tabular}

\subsection{Establishing benchmarks}

The data discussed above were used to establish building energy benchmarks for the three building types and for each climate zone. These benchmarks separated buildings into three groups based upon their EUI and relationship to their peers. "Good" or "Green" buildings were those with EUIs in the first quartile of all buildings with the same criteria; "Bad" or "Red" buildings were those above the third quartile; and "Acceptable" or "Amber" buildings were those between the first and third quartile. Figure 27, Figure 28, and Figure 29 show the three benchmarking categories for barracks, DFACs, and vehicle maintenance buildings, respectively. Table 21, Table 22, and Table 23 provide the exact quartile values for each climate zone and building type. Within the quartile charts, the bottom whisker highlights $25 \%$ of the buildings with the lowest EUIs; the top whisker represents $25 \%$ of the buildings with the worst EUIs; and the middle amber box contains the remaining $50 \%$ of buildings. The dark line within the box is representative of the median of the dataset. 
The quartile charts show that the variability of the data differs considerably between building types and locations. Depending on location, the three benchmarking classifications may have smaller or larger EUI ranges. Therefore, a small change in a building's EUI could cause a shift in its performance rating or a large shift could have a negligible impact.

Quartiles were selected as the benchmarking criteria because they provide a quantitative means for gauging where a target building stands in comparison to similar buildings. Quartiles can also be dynamically changed as building energy performance improves. This enables users to rapidly shift the Good, Bad, and Acceptable standards as necessary to accommodate changes in consensus baselines or revisions of government or industry standards.

Additionally, quartile ratings can be easily automated and integrated into additional energy-modeling software. In this study, the benchmarks were used to inform the BUILDERTM Sustainment Management System ${ }^{11}$ Energy module as to whether an energy-efficiency measure (EEM) or set of EEMs would allow buildings under analysis to meet or exceed peer norms. Such analyses can help building managers with determining how best to improve their building's energy performance.

\footnotetext{
11 The BULDERTM Sustainment Management System (SMS) is a web-based software application developed by ERDC-CERL to support the DoD facilities management community optimize infrastructure maintenance planning, scheduling, and resource allocation. More information about BUILDERTM is available at http://www.erdc.usace.army.mil/Media/Fact-Sheets/Fact-Sheet-Article-View/Article/476728/builder-sustainment-management-system/
} 
Figure 27. Barracks benchmark quartiles.

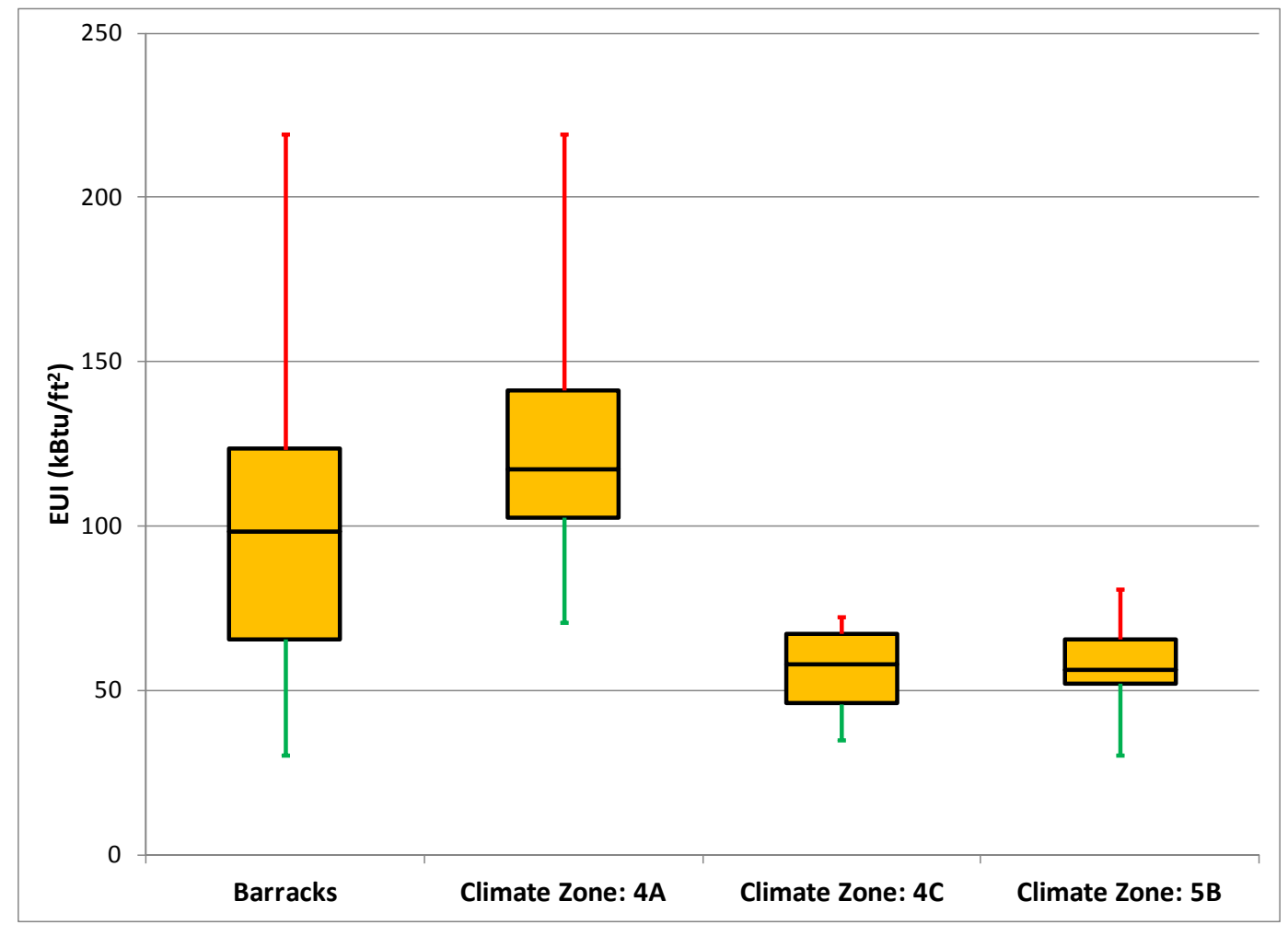

Table 21. Barracks quartile values.

\begin{tabular}{|c|r|r|r|r|}
\hline EUls (kBtu/ft ${ }^{2}$ ) & Barracks & Climate Zone: 4A & Climate Zone: 4C & Climate Zone: 5B \\
\hline Minimum & 30.34 & 70.85 & 34.80 & 30.34 \\
\hline 1st Quartile & 65.64 & 102.69 & 46.06 & 52.10 \\
\hline Median & 98.32 & 117.45 & 57.72 & 56.24 \\
\hline 3rd Quartile & 123.41 & 141.29 & 67.29 & 65.67 \\
\hline Maximum & 219.25 & 219.25 & 72.25 & 80.95 \\
\hline
\end{tabular}


Figure 28. DFAC benchmark quartiles.

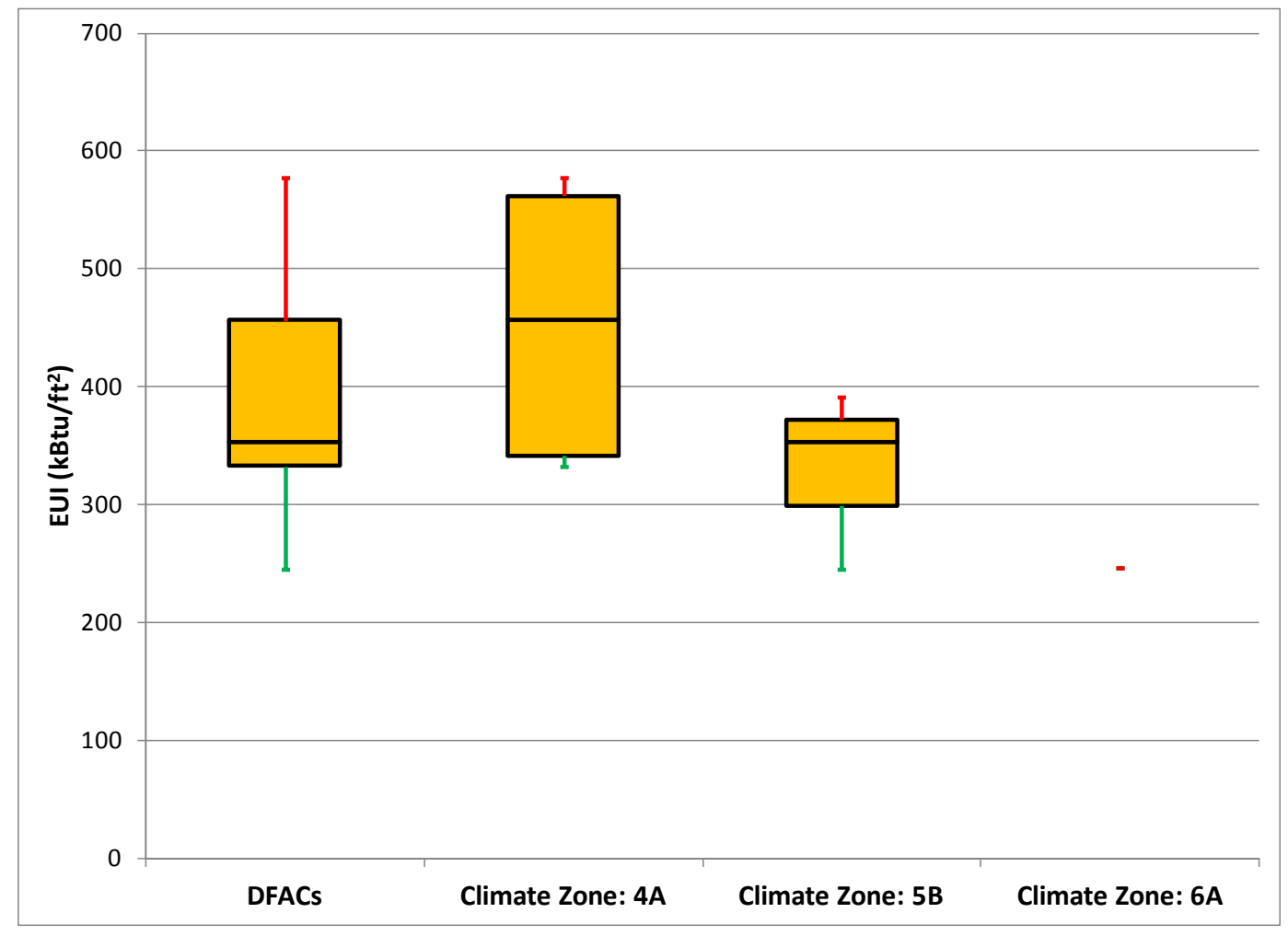

Table 22. DFAC quartile values.

\begin{tabular}{|c|r|r|r|r|}
\hline EUls (kBtu/ft ${ }^{2}$ ) & \multicolumn{1}{|c|}{ DFACs } & Climate Zone: 4A & Climate Zone: 5B & Climate Zone: 6A \\
\hline Minimum & 245.20 & 332.72 & 245.20 & N/A \\
\hline 1st Quartile & 332.72 & 341.34 & 299.07 & N/A \\
\hline Median & 352.95 & 456.30 & 352.95 & N/A \\
\hline 3rd Quartile & 456.30 & 561.99 & 372.25 & $\mathrm{~N} / \mathrm{A}$ \\
\hline Maximum & 577.14 & 577.14 & 391.56 & $\mathrm{~N} / \mathrm{A}$ \\
\hline
\end{tabular}


Figure 29. Vehicle maintenance benchmark quartiles.

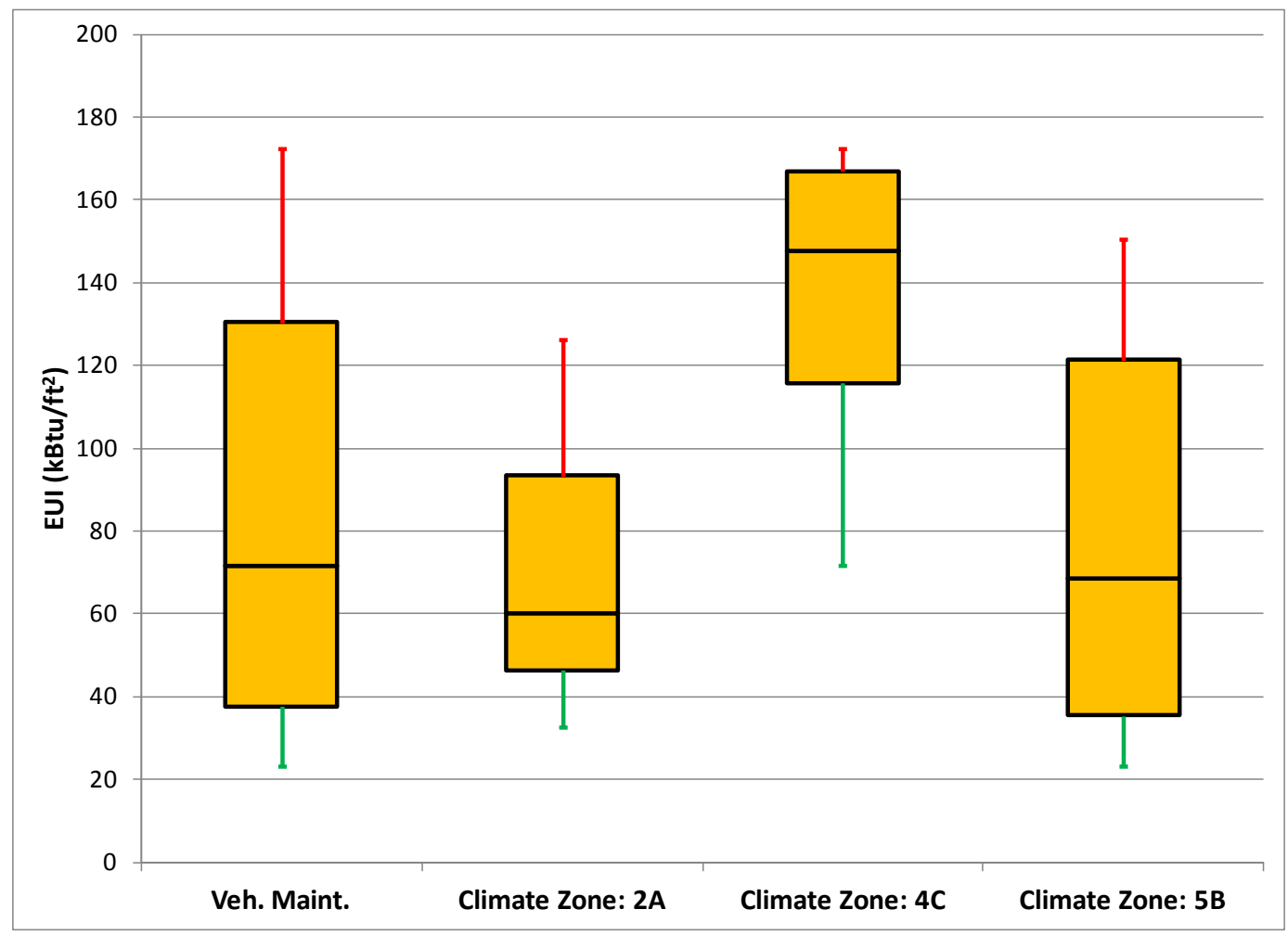

Table 23. Vehicle maintenance quartile values.

\begin{tabular}{|c|r|r|r|r|}
\hline EUls (kBtu/ft ${ }^{2}$ ) & Veh. Maint. & Climate Zone: 2A & Climate Zone: 4C & Climate Zone: 5B \\
\hline Minimum & 23.25 & 32.55 & 71.68 & 23.25 \\
\hline 1st Quartile & 37.68 & 46.40 & 115.75 & 35.51 \\
\hline Median & 71.68 & 60.24 & 147.78 & 68.53 \\
\hline 3rd Quartile & 130.44 & 93.32 & 166.96 & 121.35 \\
\hline Maximum & 172.49 & 126.40 & 172.49 & 150.61 \\
\hline
\end{tabular}

\subsection{Comparison of Army data and commercial studies}

The data presented above can be compared with the results of commercial studies at other locations. Table 24 compares the median EUIs of six additional studies with those calculated here. The commercial building types used to calculate medians in each study are provided in Table 25. An overview of each study is provided below. 
Table 24. Commercial studies building EUls.

\begin{tabular}{|c|r|r|r|}
\hline Annual Median EUIs (kBtu/ft ${ }^{\mathbf{}}$ ) & \multicolumn{1}{|c|}{ Barracks } & Dining Facilities & Vehicle Maintenance \\
\hline CBECS - 2003 & 73.90 & 228.50 & 49.30 \\
\hline CTS & 65.50 & 146.10 & 189.60 \\
\hline DC - 2013 & 50.40 & -- & --20 \\
\hline NYC - 2013 & 84.20 & 213.50 & - \\
\hline CEUS & 83.78 & 347.15 & -- \\
\hline CEC & 154.00 & 712.00 & 71.68 \\
\hline Army & 98.32 & 352.95 & 98.70 \\
\hline Mean & 85.30 & 329.45 & 62.30 \\
\hline Standard Deviation & 35.95 & 225.78 & 0.43 \\
\hline Army Standard Deviation from Mean & 0.30 & 0.10 & \\
\hline
\end{tabular}

Table 25. Commercial study building datasets.

\begin{tabular}{|c|c|c|c|}
\hline Commercial Studies & Barracks & Dining Facilities & Vehicle Maintenance \\
\hline CBECS - 2003 & $\begin{array}{l}\text {-Dormitory } \\
\text { - Fraternity } \\
\text { - Sorority } \\
\end{array}$ & $\begin{array}{l}\text { - Restaurant } \\
\text { - Cafeteria }\end{array}$ & $\begin{array}{l}\bullet \text { Vehicle } \\
\text { service/repair shop }\end{array}$ \\
\hline CTS & $\begin{array}{l}\bullet \text { Residence } \\
\text { Hall/Dormitory }\end{array}$ & $\begin{array}{l}\text { - Food service } \\
\text { - Restaurant/cafeteria }\end{array}$ & $\begin{array}{l}\text { Vehicle } \\
\text { service/repair } \\
\text {-Vehicle } \\
\end{array}$ \\
\hline DC - 2013 & $\begin{array}{l}\bullet \text { Residence } \\
\text { Hall/Dormitory }\end{array}$ & N/A & N/A \\
\hline NYC - 2013 & $\begin{array}{l}\bullet \text { Residence } \\
\text { Hall/Dormitory }\end{array}$ & $\begin{array}{l}\text { - Restaurant } \\
\text { - Food service }\end{array}$ & $\begin{array}{l}\text {-Automobile } \\
\text { dealership }\end{array}$ \\
\hline CEUS & -Lodging & -Restaurant & $N / A$ \\
\hline CEC & - Lodging, Motel & •"Other" restaurant & $N / A$ \\
\hline
\end{tabular}

\subsubsection{Commercial Buildings Energy Consumption Survey (CBECS) ${ }^{12}$}

The Commercial Buildings Energy Consumption Survey (CBECS) is a national sample survey that collects information on the stock of U.S. commercial buildings, including their energy-related building characteristics and energy usage data (consumption and expenditures). Commercial buildings in this definition includes all buildings in which at least half of the floor space is used for a purpose that is not residential, industrial, or agricultural. This includes schools, hospitals, and correctional institutions, in addition to "traditional" commercial building uses.

\footnotetext{
12 U.S. Energy Information Administration - EIA - Independent Statistics and Analysis. (n.d.). Retrieved November 13, 2015, from http://www.eia.gov/consumption/commercial/
} 
CBECS is administered by the U.S. Department of Energy's (DOE) Energy Information Administration (EIA). The EIA was created in 1978, along with the DOE, after the 1973 oil embargo revealed a need to cut the nation's dependence on foreign oil. Its primary mission is to gather data on U.S. energy supply and demand. The EIA conducted its first CBECS in 1979, followed by surveys in 1983, 1986, 1989, 1992, 1995, 1999, and 2003. The upcoming 2012 CBECS, due to roll out in 2016, will be the ninth iteration. ${ }^{13}$

\subsubsection{EISA 432 Compliance Tracking System (CTS)}

The CTS is the repository for all federal benchmarking efforts (including MDMS output). It is overseen by the U.S. Department of Energy Office of Energy Efficiency and Renewable Energy. The CTS consists of five data sets that can be used to build custom reports (1) facility annual detail, (2) comprehensive evaluation detail, (3) benchmarked building detail, (4) project and follow-up detail and, the most recent, (5) facility overview. ${ }^{1415}$

\subsubsection{Washington, DC}

Data disclosures are divided into public and private buildings. Public results are published through the District Department of the Environment (DDOE) and the Department of General Services (DGS) and include 15minute interval data for electricity use. The private building results are published by the District of Columbia annually, and include energy and water performance benchmarking data from the city's largest buildings. ${ }^{16}$

\subsubsection{New York City, NY}

Data disclosures are divided into public and private buildings. Both public and private energy benchmarking uses Portfolio Manager, which is cited as being "widely accepted as the industry standard for benchmarking."

\footnotetext{
13 Maria Gallucci, InsideClimate News, National Building Audit to Reset Crucial Energy Use Benchmarks, 7 March 2012, http://insideclimatenews.org/news/20120307/national-building-audit-reset-crucialenergy-use-benchmarks.

1442 U.S.C.A. § 8253 (West).

15EISA Federal Facility Management and Benchmarking Reporting Requirements. (n.d.). Retrieved November 13, 2015, from http://energy.gov/eere/femp/eisa-federal-facility-management-and-benchmarking-reporting-requirements.

16 Energy Benchmarking Disclosure. (n.d.). Retrieved November 13, 2015, from http://doee.dc.gov/page/energy-benchmarking-disclosure.
} 
Public buildings are monitored by the Department of Citywide Administrative Services (DCAS) and include total electricity, natural gas, district steam, and heating fuel oil. Approximately 4,000 public buildings have been benchmarked since 2009. Owners of large, private buildings are mandated by Local Law 84 to annually measure their energy and water consumption. ${ }^{17}$

\subsubsection{California Commercial End-Use Survey (CEUS)}

CEUS is a study of commercial sector energy use, primarily collected to provide data for energy demand forecasting. CEUS consists of a "stratified random sample" of 2,790 commercial facilities. It is overseen by the California Energy Commission (CEC). Utility service area, floor stocks, fuel shares, electric/ natural gas consumption, EUIs, energy intensities, and 16day hourly end-use load profiles were estimated for twelve common commercial building types. ${ }^{18}$

\subsubsection{Nonresidential Building Energy Use Disclosure Program (CEC)}

The Nonresidential Building Energy Use Disclosure Program encourages greater energy efficiency in nonresidential buildings by requiring building owners to disclose energy use to prospective buyers, lessees, and lenders, as well as to the California Energy Commission. This statewide program applies in addition to benchmarking and disclosure programs already in place and under development in local jurisdictions. ${ }^{19}$

\subsubsection{Summary of comparisons}

These studies demonstrate that the Army energy usage is comparable to that reported in some commercial studies but quite different from others. Therefore, it cannot be stated conclusively that commercial studies are applicable to Army installations without further analysis. The difference between Army and commercial building occupancy and usage characteristics

\footnotetext{
17 Benchmarking Data Disclosure \& Reports. (n.d.). Retrieved November 13, 2015, from http://www.nyc.gov/html/gbee/html/plan/ll84_scores.shtml.

18 Itron Inc. March 2006. California Commercial End-Use Survey. California Energy Commission. Retrieved November 13, 2015, from http://www.energy.ca.gov/2006publications/CEC-400-2006005/CEC-400-2006-005.PDF.

19 Nonresidential Building Energy Use Disclosure Program (AB 1103). (n.d.). Retrieved November 13, 2015, from http://www.energy.ca.gov/ab1103/.
} 
likely account for some of these discrepancies. While commercial buildings tend to have well regimented occupancy patterns and limited variability, Army building occupancy can change drastically as a result of deployment and training schedules. Such activities often leave Army buildings underutilized.

Additionally, it proved difficult to find commercial counterparts for Army buildings. As shown in Table 25, there are no exact matches between Army and commercial buildings. Although a dormitory, motel, or other commercial lodging facilities may have similarities with barracks buildings, the usage and occupancy patterns are not identical. Similarly, an automobile dealership differs considerably from an Army vehicle maintenance facility. Unfortunately, the building types listed in Table 25 are the closest approximations for the Army buildings studied here. Therefore, it is not surprising that Army EUIs differ from those of commercial buildings addressed in other commercial studies. Further study would be required to determine the exact occupancy, usage, and construction differences between Army and commercial buildings and how they can be accounted for when comparing the two different standards. 


\section{Conclusions and Recommendations}

\subsection{Conclusions}

While Army energy data remain scarce and commercial studies are inconclusive, the analysis presented in Chapter 4 provides usable benchmarks for BUILDER and energy managers. Additional data and further studies could be used to refine the present results, but currently available data highlight where Army energy usage differs from similar commercial buildings and which buildings consume a disproportionate amount of energy. As more buildings are metered and additional energy-consumption factors are studied, the sensitivity and clarity of these benchmarks will improve.

\subsection{Recommendations}

\subsubsection{Improve data in Army real property databases}

\subsubsection{Populate existing building attributes in HQIIS and GFEBS}

Accurate populating and updating of HQIIS and GFEBS data fields would greatly facilitate benchmarking efforts currently under way by the Army. Useful data fields that are often not populated are shown in Table 26. Understanding the physical makeup of a building is fundamental to rigorous benchmarking.

Table 26. Existing building attributes in HQIIS that are often not populated with data.

\begin{tabular}{|l|l|}
\hline Structure construction material code & FCLTY_STRUCT_CONST_MATL_CD \\
\hline Foundation construction material code & FCLTY_FNDTN_CONST_MATL_CD \\
\hline Floor construction material code & FCLTY_FLOOR_CONST_MATL_CD \\
\hline Wall construction material code & FCLTY_WALL_CONST_MATL_CD \\
\hline Roof construction material code & FCLTY_ROOF_CONST_MATL_CD \\
\hline
\end{tabular}

\subsubsection{Add new building attributes to HQIIS and GFEBS}

In an effort to hone Army benchmarks, it is recommended that more building attributes be added to existing Army and DoD real property databases. Recommendations are summarized in Table 27. The legacy Integrated Facilities System (IFS) database incorporated data fields that would benefit energy benchmarking activities, namely those that described physical attributes of the facilities such as those shown in Table 28. 
Table 27. Building attributes for Army to consider tracking in the future (EIA).

\begin{tabular}{|l|l|l|}
\hline Facility shape & $\begin{array}{l}\text { Vacancy status (building } \\
\text { hours, occupancy) }\end{array}$ & $\begin{array}{l}\text { Conveyance (elevators, } \\
\text { escalators, moving walks) }\end{array}$ \\
\hline Roof type / pitch & Renovations before 1980 & Window-to-wall ratio (all sides) \\
\hline Attic $(\mathrm{y} / \mathrm{n})$ & Water heating & Refrigeration equipment \\
\hline Cool roof & Cooking equipment & Manufacturing equipment \\
\hline Lighting schedules & Office equipment & Equipment usage schedules 20 \\
\hline
\end{tabular}

Table 28. Useful attributes tracked in the IFS database.

\section{CONSTRUCTION CHARACTERISTICS}

\begin{tabular}{|c|c|}
\hline Area Total Roof & AREA_TOTAL_ROOF \\
\hline Ceiling Height Basement & CEILING_HGHT_BASEMENT \\
\hline Ceiling Height Feet & CEILING_HEIGHT_FEET \\
\hline Facility Construction Remarks & FAC_CONSTR_RMKS \\
\hline Facility Dimensions Attic & FACILITY_DIMENSIONS_ATTIC \\
\hline Facility Dimensions Basement & FACILITY_DIMENSIONS_BASEMENT \\
\hline Facility Dimensions Offset & FACILITY_DIMENSIONS_OFFSET \\
\hline Facility Dimensions Wings & FACILITY_DIMENSIONS_WINGS \\
\hline \multicolumn{2}{|l|}{ ELECTRIC } \\
\hline Electric Amperes & ELECTRIC_AMPERES \\
\hline Electric Kilovolt Amperes Rating & ELECTRIC_KVA_RATING \\
\hline Electric Phase & ELECTRIC_PHASE \\
\hline Electric Voltage & ELECTRIC_VOLTAGE \\
\hline Electric Wire Size & ELECTRIC_WIRE_SIZE \\
\hline \multicolumn{2}{|l|}{ GAS } \\
\hline Gas Pipe Pressure & GAS_PIPE_PRESSURE \\
\hline Gas Pipe Size & GAS_PIPE_SIZE \\
\hline \multicolumn{2}{|l|}{ HOT WATER } \\
\hline Hot Water Capacity & HOT_WATER_CAPACITY \\
\hline Hot Water Temperature & HOT_WATER_TEMPERATURE \\
\hline \multicolumn{2}{|l|}{ STEAM } \\
\hline Steam Pipe Pressure & STEAM_PIPE_PRESSURE \\
\hline Steam Pipe Size & STEAM_PIPE_SIZE \\
\hline
\end{tabular}

20 CBECS 2003 website, http://www.eia.gov/consumption/commercial/data/2003/\#b1 


\begin{tabular}{|c|c|}
\hline Steam Process Pipe Pressure & STEAM_PROCESS_PIPE_PRESSURE \\
\hline Steam Process Pipe Size & STEAM_PROCESS_PIPE_SIZE \\
\hline \multicolumn{2}{|l|}{ VENTILATION } \\
\hline Mechanically Ventilated Area & MECHANICALLY_VENTILATED_AREA \\
\hline \multicolumn{2}{|l|}{ HEATING } \\
\hline Heat Demand Unit & HEAT_DEMAND_UNIT \\
\hline Heated Space Cubic Feet & HEATED_SPACE_CUBIC_FEET \\
\hline Heating Capacity & HEATING_CAPACITY_MBTU \\
\hline \multicolumn{2}{|l|}{ COOLING } \\
\hline Air Conditioned Area Square Feet & AIR_CONDITIONED_AREA_SQUARE_FT \\
\hline Air Conditioner Capacity & AIR_CONDITIONER_CAPACITY \\
\hline Condensate Capacity & CONDENSATE_CAPACITY \\
\hline Condensate Size & CONDENSATE_SIZE \\
\hline Humidity Controlled Space & HUMIDITY_CONTROLLED_SPACE \\
\hline \multicolumn{2}{|l|}{ METERS } \\
\hline Meter Assignment Proration Percentage & METER_ASGMT_PRORATE_PCT \\
\hline Meter Constant Number & METER_CONSTANT_NO \\
\hline Meter Reading Count & METER_READ_COUNT \\
\hline Meter Reading Date & METER_READ_DATE \\
\hline Meter Reading Inoperative Indicator & METER_READ_INOPER_IND \\
\hline Meter Rollover Amount & METER_ROLLOVER_AMT \\
\hline \multicolumn{2}{|l|}{ MISCELLANEOUS } \\
\hline Critical Utility & CRITICAL_UTILITY \\
\hline Date Equipment Warranty & DATE_EQUIPMENT_WARRANTY \\
\hline
\end{tabular}

\subsubsection{Improve Army benchmarks}

The benchmarks established in this research were developed using the best information available at the time. Nevertheless, these benchmarking standards could be improved if steps are taken to increase the available datasets and/ or further refine data collection and analysis techniques, as suggested below.

\subsubsection{Benchmark more Army building types}

The present study analyzed the energy consumption of Army building barracks, dining facilities, and vehicle maintenance buildings. To obtain a clear understanding of building energy consumption, however, many other 
types of buildings also must be analyzed. EPAct 2005 requires the metering of all federal buildings where economically practicable. ${ }^{21}$ This has been interpreted by the Army as all buildings larger than 29,000 $\mathrm{ft}^{2} 22$. Among such large buildings, some are more common and critical than others and several are known to have particularly high EUIs. The buildings in Table 29 fall into this category and should be studied in accordance with their priority. They have been prioritized based on total floor area of the particular building type across all Army installations.

Table 29. Building priority for future benchmarking studies.

\begin{tabular}{|c|c|c|c|c|}
\hline Priority & $\begin{array}{c}\text { Total Floor } \\
\text { Area }\left(\mathrm{ft}^{2}\right)\end{array}$ & $\begin{array}{l}\text { Quantity } \\
\text { (Q3 FY15) }\end{array}$ & CATCODE & Description \\
\hline \multirow{2}{*}{1} & $61,749,142$ & 619 & 61050 & General Administrative Building (ADMIN GEN PURP) \\
\hline & $1,456,484$ & 34 & 61001 & General Administrative Building (MEPS) \\
\hline \multirow{2}{*}{2} & $15,521,513$ & 249 & 21110 & Aircraft Maintenance Hangar \\
\hline & 907,047 & 20 & 21114 & Aircraft Maintenance Hangar (AC MAINT BAY) \\
\hline 3 & $15,947,268$ & 44 & 51010 & Hospital (MED CTR/HOSP) \\
\hline 4 & $13,429,900$ & 165 & 73046 & Dependent School \\
\hline 5 & $12,846,500$ & 261 & 14185 & Small Unit Headquarters Building (CO HQ) \\
\hline 6 & $11,628,508$ & 144 & 17120 & General Purpose Instruction Building \\
\hline 7 & $5,607,701$ & 64 & 74053 & Exchange Sales Facility (EXCH MAIN STORE) \\
\hline 8 & $5,199,001$ & 67 & 74021 & Commissary \\
\hline 9 & $4,568,022$ & 54 & 14133 & Operations Supply Building (SHIP/RECV FAC) \\
\hline 10 & $4,319,374$ & 30 & 14162 & Emergency Operations Center / SCIF \\
\hline
\end{tabular}

Additionally, the following buildings provide an excellent opportunity for future benchmarking studies owing to data collected in previous studies. By leveraging these data, more accurate benchmarking models could be generated. Army installation personnel could use these better understand the energy consumption of a greater variety of building types. Benchmarking models are recommended for the following building types:

- Army Reserve Center (ARC)

- Brigade Headquarters (BdeHQ)

- Battalion Headquarters (BnHQ)

- Child Development Center (CDC)

- Company Operations Facility (COF)

- General Instruction Building (GIB)

- General Purpose Warehouse (GPW)

\footnotetext{
21 Public Law 109-58; DOE/EE-0312, p ii.

22 Valine, Debra, “Corps of Engineers helps Army installations reduce energy use, save money", USACE Huntsville Center, August 31, 2009, http://www.army.mil/mobile/article/?p=26770.
} 
- Information Systems Facility (InfoSys)

- Outpatient Healthcare Center (OHC)

\subsubsection{Benchmark refinements}

As more meters are added, larger datasets will enable more rigorous statistical analysis of Army EUIs, thereby improving benchmarking standards. As more installations and buildings are metered, development of installation-specific benchmarks that better reflect local missions and operations can begin.

Submetering is required to fully comply with the reporting requirements established in 10 C.F.R. § 433. Subsection 433 states:

\footnotetext{
"Energy consumption for the purposes of calculating the 30 percent savings shall include space heating, space cooling, ventilation, service water heating, lighting and all other energy consuming systems normally specified as part of the building design except for receptacle and process loads."23
}

Only by submetering can process loads be eliminated from current building energy consumption figures as required by Subsection 433. Furthermore, submetering will increase the specificity and accuracy of benchmarking standards. For example, energy usage, such as that required for cooking in a DFAC, can be accounted for and removed from the overall energy consumption of the building. Since this load is ancillary to the energy requirements for the building, it is unnecessarily included in current energy consumption calculations. Ideally, building benchmarks would only account for building energy consumption independent of the activities within the building.

\subsubsection{Weather normalization}

As specified previously, this study did not normalize for weather-related changes in building energy consumption. Due to the logistical challenges of obtaining weather data at each location and a lack of 15 minute weather data at many locations, weather normalization proved to be infeasible. Nevertheless, the importance of such an analysis is not ignored. Currently,

\footnotetext{
2310 C.F.R. $\S 433.1$
} 
the Illinois Smart Energy Design Assistance Center (SEDAC), in partnership with ERDC-CERL, is attempting to better characterize building energy use patterns. Studying the effect of weather conditions on building energy consumption is a significant step toward this goal. During this effort, for barracks in climate zone 4A, SEDAC is attempting to identify weather-dependent behaviors and nondependent behaviors; study nondependent behaviors; and identify facility components that drive such behaviors. Results are forthcoming.

\subsubsection{Improve MDMS}

Currently, MDMS data is highly dependent upon installation-specific reporting standards. As a result, some data are reported directly and accurately to MDMS while other data are manually uploaded in batches. This discrepancy can impact data quality and yield substandard or incomplete information. It is recommended that MDMS establish a means of verifying all uploaded data and that clear protocols be implemented for data recording, delivery, and analysis.

Clear building-type identification would also significantly improve the usability of the system. By identifying buildings using the DoD Real Property Categorization System (RPCS) category codes (CatCodes) in addition to their names, the system could be more easily organized, maintained, and queried.

Additionally, a column should be added to the MDMS database screen that states cumulative energy consumption. The system currently provides only interval data. Cumulative data would provide greater flexibility for data analysis. Separating the data into monthly and annual consumption figures would further increase the usability and practicality of the system. 


\section{References}

California Energy Commission. (n.d.). Nonresidential Building Energy Use Disclosure Program, Assembly Bill (AB) 1103. http://www.energy.ca.gov/ab1103/

Department of Defense. November 1993. Defense Utility Energy Reporting System (DUERS) Manual. DoD 5126-46-M-2, p 1-1.

Department of Energy and Environment. (n.d.) "Energy Benchmarking Disclosure." City of Washington, DC website. Retrieved 13 November 2015. http://doee.dc.gov/page/energy-benchmarking-disclosure

Energy Efficiency and Renewable Energy (EERE). December 2010. "Advanced Metering Solutions for Federal Agencies." Meeting Report at Washington Convention Center. Washington, DC: U.S. Department of Energy. www1 eere.energy.gov.

Energy Independence and Security Act (EISA) Federal Facility Management and Benchmarking Reporting Requirements. (n.d.).http://energy.gov/eere/femp/eisafederal-facility-management-and-benchmarking-reporting-requirements

Energy Information Administration (EIA). 2003. “Commercial Buildings Energy Consumption Survey (CBECS).” Washington, DC: U.S. Department of Energy. http://www.eia.gov/consumption/commercial/data/2003/\#b1

Energy Information Administration (EIA). December 2015. "Select Results from the Energy Assessor Experiment in the 2012 Commercial Buildings Energy Consumption Survey." Washington, DC: U.S. Department of Energy. http://www.eia.gov/consumption/commercial/

Ford, Charles. 2007. "Huntsville Center Projects Range from Saving Energy to Designing State-of-the-Art Facilities.” Public Works Digest Vol. XIX, No. 6, pp 9-11.

Gallucci, Maria. 2012. "National Building Audit to Reset Crucial Energy Use Benchmarks." Brooklyn, NY: InsideClimate News. http://insideclimatenews.org/news/20120307/national-building-audit-reset-crucial-energyuse-benchmarks.

Intuitive Research and Technology Corporation. 2005. Department of Defense Energy Manager's Handbook ( $p$ 43). Washington, DC: Department of Defense.

Itron Inc. March 2006. California Commercial End-Use Survey. Consultant Report for California Energy Commission. http://www.energy.ca.gov/2006publications/CEC-4002006-005/CEC-400-2006-005.PDF

J osefik, Nicholas M., J ulie L. Webster, Shane D. Hirschi, Matthew D. Hiett, and Sanat S. Bhole. August 2017. Legal and Administrative Requirements for Energy-Use Benchmarking and Metering of Military Facilities. ERDC/ CERL SR-17-13. Champaign, IL: U.S. Army Engineer Research and Development Center, Construction Engineering Research Laboratory. 
NYC Mayor's Office of Sustainability. (n.d.). Benchmarking Data Disclosure and Reports LL84. Retrieved November 13, 2015

http://www.nyc.gov/html/gbee/html/plan/ll84_scores.shtml

Public Law 10 C.F.R. § 433.1

Public Law 109-58. Energy Policy Act of 2005 (EPAct 2005).

http://www.gpo.gov/fdsys/pkg/PLAW-109publ58/pdf/PLAW-109publ58.pdf

Public Law 109-58; DOE/EE-0312, p ii.

Public Law 110-140. Energy Independence and Security Act (EISA). 19 December 2007. http://www.gpo.gov/fdsys/pkg/BILLS-110hr6enr/pdf/BILLS-110hr6enr.pdf

Public Law 42 U.S.C. § 8253. Energy management requirements.

Valine, Debra. August 2009. "Corps of Engineers helps Army installations reduce energy use, save money," Huntsville, AL: USACE Huntsville Center.

http://www.army.mil/mobile/article/?p=26770 


\section{Appendix: Building Attributes Used in Data Analyses}

Table A1. Analyzed data for barracks (possible outlier highlighted). 24

\begin{tabular}{|c|c|c|c|c|c|c|}
\hline Bldg ID & \begin{tabular}{|l|} 
Climate Zone \\
\end{tabular} & Area $\left(\mathrm{ft}^{2}\right)$ & Construction Type & Construction Year & \begin{tabular}{|l|} 
Data Year(s) \\
\end{tabular} & EUI $\left(\mathrm{kBtu} / \mathrm{ft}^{2}\right)$ \\
\hline B1 & $4 \mathrm{~A}$ & 40,640 & Brick Veneer & 1964 & 2013 & 100.28 \\
\hline B2 & $4 \mathrm{~A}$ & 40,990 & Brick Veneer & 1963 & 2013 & 113.51 \\
\hline B3 & $4 \mathrm{~A}$ & 40990 & Brick Veneer & 1961 & 2013-2014 & 117.97 \\
\hline B4 & $4 \mathrm{~A}$ & 40,990 & Brick Veneer & 1961 & 2014 & 109.80 \\
\hline B5 & $4 \mathrm{~A}$ & 40,640 & Brick Veneer & 1965 & 2014 & 105.10 \\
\hline B6 & $4 \mathrm{~A}$ & 40,640 & Brick Veneer & 1965 & 2014 & 80.02 \\
\hline B7 & $4 \mathrm{~A}$ & 40,640 & Brick Veneer & 1967 & 2014 & 167.90 \\
\hline B8 & $4 \mathrm{~A}$ & 40,749 & Brick Veneer & 1961 & 2014 & 110.50 \\
\hline B9 & $4 \mathrm{~A}$ & 40,749 & Brick Veneer & 1961 & 2014 & 123.41 \\
\hline B10 & $4 \mathrm{~A}$ & 40,749 & Brick Veneer & 1961 & 2014 & 128.43 \\
\hline B11 & $4 \mathrm{~A}$ & 40,749 & Brick Veneer & 1961 & 2014 & 123.75 \\
\hline B12 & $4 \mathrm{~A}$ & 43,718 & Brick Veneer & 1967 & 2014 & 93.92 \\
\hline B13 & $4 \mathrm{~A}$ & 43,718 & Brick Veneer & 1967 & 2014 & 153.33 \\
\hline B14 & $4 \mathrm{~A}$ & 43,718 & Brick Veneer & 1967 & 2014 & 181.88 \\
\hline B15 & $4 \mathrm{~A}$ & 43,718 & Brick Veneer & 1967 & 2014 & 182.30 \\
\hline B16 & $4 \mathrm{~A}$ & 55,448 & Brick Veneer & 2010 & 2014 & 147.19 \\
\hline B17 & $4 \mathrm{~A}$ & 55,448 & Brick Veneer & 2010 & 2014 & 117.45 \\
\hline B18 & $4 \mathrm{~A}$ & 55,448 & Brick Veneer & 2010 & 2014 & 135.40 \\
\hline B19 & $4 \mathrm{~A}$ & 55,448 & Brick Veneer & 2012 & 2014 & 105.11 \\
\hline $\mathrm{B} 20$ & $4 \mathrm{~A}$ & 55,448 & Brick Veneer & 2011 & 2014 & 98.32 \\
\hline B21 & $4 \mathrm{~A}$ & 55,448 & Brick Veneer & 2012 & 2014 & 77.79 \\
\hline B22 & $4 \mathrm{~A}$ & 55,448 & Brick Veneer & 2012 & 2014 & 70.85 \\
\hline B23 & $4 \mathrm{~A}$ & 43,718 & Brick Veneer & 1967 & 2014 & 219.25 \\
\hline B23 & $4 C$ & 105,852 & Brick Veneer & 1927 & 2014 & 34.80 \\
\hline B24 & $4 \mathrm{C}$ & 50,768 & Other & 1957 & 2014 & 72.25 \\
\hline B25 & $4 C$ & 50,768 & Other & 1957 & 2014 & 65.64 \\
\hline B26 & $4 \mathrm{C}$ & 107,225 & Brick Veneer & 1927 & $2013-2014$ & 49.81 \\
\hline B27 & $5 B$ & 49,560 & Concrete & 2007 & 2014 & 75.41 \\
\hline B28 & $5 B$ & 95,858 & Block & 1998 & 2014 & 30.34 \\
\hline B29 & $5 B$ & 123,860 & Block & 2004 & 2014 & 80.95 \\
\hline $\mathrm{B} 30$ & $5 B$ & 123,860 & Block & 2004 & 2012 & 64.35 \\
\hline B31 & $5 B$ & 95,858 & Brick Veneer & 1998 & 2012 & 66.11 \\
\hline B32 & $5 B$ & 152,684 & Combo: Wood/Masonry Frame & 2009 & 2014 & 53.64 \\
\hline B33 & $5 B$ & 152,684 & Combo: Wood/Masonry Frame & 2009 & 2014 & 51.58 \\
\hline B34 & $5 B$ & 152,684 & Combo: Wood/Masonry Frame & 2009 & 2014 & 54.19 \\
\hline B35 & $5 B$ & 152,684 & Prefab & 2009 & 2014 & 58.29 \\
\hline B36 & $5 B$ & 152,684 & Prefab & 2009 & 2014 & 34.09 \\
\hline
\end{tabular}

24 Highlighted rows in Tables 25, 26, and 27 indicate potential outliers. Neither building proved to be an outlier after further analysis. See Section 4.4. 
Table A2. Analyzed data for dining facilities (possible outlier highlighted). 25

\begin{tabular}{|c|c|c|c|c|c|c|}
\hline BIdg ID & Climate Zone & Area $\left(\mathbf{f t}^{\mathbf{}}\right.$ ) & Construction Type & Construction Year & Data Year(s) & EUI (kBtu/ft ${ }^{2}$ ) \\
\hline D1 & 4A & 27,263 & Brick Veneer & 2012 & $2013-2014$ & 456.30 \\
\hline D2 & 4A & 20,580 & Brick Veneer & 1999 & $2013-2014$ & 561.99 \\
\hline D3 & 4A & 13,644 & Brick Veneer & 1966 & $2014-2015$ & 332.72 \\
\hline D4 & 4A & 13,280 & Brick Veneer & 1966 & $2014-2015$ & 577.14 \\
\hline D5 & 4A & 25,530 & Brick Veneer & 1967 & $2014-2015$ & 341.34 \\
\hline D6 & 5B & 26,500 & Concrete & 2011 & $2012-2013$ & 245.20 \\
\hline D7 & 5B & 26,500 & Curtain Wall & 2009 & $2012-2013$ & 352.95 \\
\hline D8 & 5B & 28,621 & Block & 2004 & 2014 & 391.56 \\
\hline D9 & 6A & 13,939 & Brick Veneer & 2004 & $2013-2014$ & 246.19 \\
\hline
\end{tabular}

Table A3. Analyzed data for vehicle maintenance buildings (possible outlier highlighted). ${ }^{26}$

\begin{tabular}{|c|c|c|c|c|c|c|}
\hline Bldg ID & Climate Zone & Area $\left(\mathrm{ft}^{2}\right)$ & Construction Type & Construction Year & Data Year(s) & EUI $\left(\mathrm{kBtu} / \mathrm{ft}^{2}\right)$ \\
\hline V1 & $2 A$ & 6,435 & Unknown & 1983 & 2013 & 126.40 \\
\hline $\mathrm{V} 2$ & $2 \mathrm{~A}$ & 15,466 & Unknown & 1983 & 2013 & 60.24 \\
\hline V3 & $2 \mathrm{~A}$ & 22,823 & Unknown & 1982 & 2013 & 32.55 \\
\hline V4 & $4 C$ & 14,617 & Other & 1942 & 2014 & 172.49 \\
\hline V5 & $4 C$ & 28,400 & Other & 1988 & 2014 & 130.44 \\
\hline V6 & $4 C$ & 37,147 & Block & 1959 & 2014 & 71.68 \\
\hline V7 & $4 C$ & 15,726 & Other & 1939 & 2014 & 165.12 \\
\hline V8 & $5 B$ & 37,039 & Block & 1993 & 2014 & 148.92 \\
\hline V9 & $5 B$ & 23,575 & Block & 1965 & 2014 & 88.46 \\
\hline V10 & $5 B$ & 27,175 & Block & 1966 & 2014 & 44.36 \\
\hline V11 & $5 B$ & 23,575 & Block & 1966 & 2012-2013 & 37.68 \\
\hline V12 & $5 B$ & 34,571 & Concrete & 2009 & 2014 & 69.97 \\
\hline V13 & $5 B$ & 34,571 & Concrete & 2009 & 2014 & 84.63 \\
\hline V14 & $5 B$ & 23,575 & Block & 1966 & 2014 & 34.65 \\
\hline V15 & $5 B$ & 23,575 & Block & 1966 & 2014 & 32.29 \\
\hline V16 & $5 B$ & 23,575 & Block & 1967 & 2014 & 25.27 \\
\hline V17 & $5 B$ & 23,575 & Block & 1967 & 2012-2013 & 34.78 \\
\hline V18 & $5 B$ & 34,571 & Concrete & 2009 & 2012-2013 & 67.09 \\
\hline V19 & $5 B$ & 16,386 & Block & 1995 & 2014 & 23.25 \\
\hline V20 & $5 B$ & 254,000 & Block & 1974 & 2014 & 54.23 \\
\hline V21 & $5 B$ & 45,200 & Other & 1983 & 2014 & 150.61 \\
\hline V22 & $5 B$ & 28,362 & Other & 1984 & 2014 & 144.75 \\
\hline V23 & $5 B$ & 34,128 & Concrete & 2010 & 2014 & 102.41 \\
\hline V24 & $5 B$ & 34,128 & Concrete & 2010 & 2014 & 127.66 \\
\hline V25 & $5 B$ & 27,949 & Block & 1987 & 2014 & 141.57 \\
\hline
\end{tabular}

25 Highlighted rows in Tables 25, 26, and 27 indicate potential outliers. Neither building proved to be an outlier after further analysis. See Section 4.4.

26 Highlighted rows in Tables 25, 26, and 27 indicate potential outliers. Neither building proved to be an outlier after further analysis. See Section 4.4. 


\section{Abbreviations and Acronyms}

\begin{tabular}{|c|c|}
\hline ASHRAE & $\begin{array}{l}\text { American Society of Heating, Refrigerating and Air Conditioning } \\
\text { Engineers }\end{array}$ \\
\hline AEWRS & Army Energy and Water Reporting System \\
\hline ARC & Army Reserve Center \\
\hline BdeHQ & Brigade Headquarters \\
\hline BnHQ & Battalion Headquarters \\
\hline BTU & British Thermal Unit \\
\hline CatCodes & Category Codes \\
\hline CBECS & Commercial Buildings Energy Consumption Survey \\
\hline $\mathrm{CDC}$ & Child Development Center \\
\hline CEC & California Energy Commission \\
\hline CEHNC & U.S. Army Engineering and Support Center, Huntsville, Alabama \\
\hline CERL & Construction Engineering Research Laboratory \\
\hline CEUS & California Commercial End-Use Survey \\
\hline $\mathrm{COF}$ & Company Operations Facility \\
\hline CTS & Compliance Tracking System \\
\hline DA & Department of the Army \\
\hline DAIS & Data Analytics and Integration Support \\
\hline DDOE & District Department of the Environment \\
\hline DEIS & Defense Energy Information System \\
\hline DFAC & Dining Facility \\
\hline DGS & Department of General Services \\
\hline DoD & Department of Defense \\
\hline DoDI & Department of Defense Instruction \\
\hline DOE & Department of Energy \\
\hline DUERS & Defense Utility Energy Reporting System \\
\hline EEIM & Enterprise Energy Information Management \\
\hline EEM & Energy Efficiency Measure \\
\hline EIA & Energy Information Administration \\
\hline EISA & Energy Independence and Security Act \\
\hline $\mathrm{EO}$ & Executive Order \\
\hline EPA & Environment Protection Agency \\
\hline EPAct & Energy Policy Act \\
\hline ERDC & Engineer Research and Development Center \\
\hline
\end{tabular}




\begin{tabular}{|c|c|}
\hline ESPC & Energy Savings Performance Contract \\
\hline EUI & Energy Use Intensity \\
\hline EXORD & Executive Order \\
\hline FY & Fiscal Year \\
\hline GFEBS & General Fund Enterprise Business System \\
\hline GIB & General Instruction Building \\
\hline GPW & General Purpose Warehouse \\
\hline HPSB & High Performance Sustainable Building(s) \\
\hline HQIIS & Headquarters Installation Information System \\
\hline HVAC & Heating, Ventilating, and Air Conditioning \\
\hline IECC & International Energy Conservation Code \\
\hline I\&E & Installations \& Environment \\
\hline IFS & Integrated Facilities System \\
\hline InfoSys & Information Systems Facility \\
\hline LINEST & Line Statistics \\
\hline $\mathrm{OHC}$ & Outpatient Healthcare Center \\
\hline $\mathrm{OMB}$ & Office of Management and Budget \\
\hline MACOM & Major Army Command \\
\hline MDMS & Meter Data Management System \\
\hline MILCON & Military Construction \\
\hline MOU & Memorandum of Understanding \\
\hline MSC & Major Subordinate Command \\
\hline NREL & National Renewable Energy Laboratory \\
\hline PRIDE & Planning Resource Infrastructure Development and Evaluation System \\
\hline RDTE & Research, Development, Test, and Evaluation \\
\hline REMIS & Real Estate Management Information System \\
\hline RFMIS & Rental Facility Management Information System \\
\hline RPCS & Real Property Categorization System \\
\hline RPIR & Real Property Inventory Reporting \\
\hline SEDAC & Smart Energy Design Assistance Center \\
\hline SEDS & States Energy Data System \\
\hline TCO & Total Cost of Ownership \\
\hline TEMF & Tactical Equipment Maintenance Facility \\
\hline VBA & Visual Basic for Applications \\
\hline
\end{tabular}




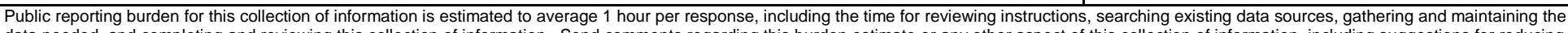

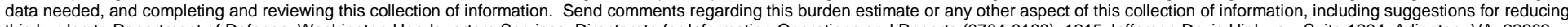

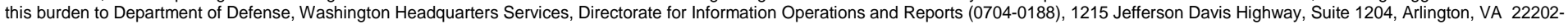

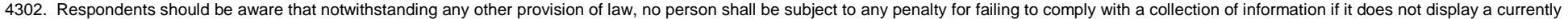
valid OMB control number. PLEASE DO NOT RETURN YOUR FORM TO THE ABOVE ADDRESS.

\begin{tabular}{|l|l|l}
\hline 1. REPORT DATE (DD-MM-YYYY) & 2. REPORT TYPE & 3. DATES COVERED (FrOm - To)
\end{tabular}

\begin{tabular}{c|c} 
August 2017 & Final \\
\hline
\end{tabular}

\section{TITLE AND SUBTITLE}

Facility Energy Performance Benchmarking in a Data-Scarce Environment

5a. CONTRACT NUMBER

5b. GRANT NUMBER

\section{5c. PROGRAM ELEMENT NUMBER}

622784T41

6. AUTHOR(S)

Sanat S. Bohle, Julie L. Webster, Matt D. Hiett, and Nicholas M. Josefik

\section{5d. PROJECT NUMBER}

P2 370256

5e. TASK NUMBER

5f. WORK UNIT NUMBER

7. PERFORMING ORGANIZATION NAME(S) AND ADDRESS(ES)

U.S. Army Engineer Research and Development Center (ERDC)

Construction Engineering Research Laboratory (CERL)

\section{PERFORMING ORGANIZATION REPORT} NUMBER

ERDC/CERL TR-17-24

PO Box 9005

Champaign, IL 61826-9005

\section{SPONSORING I MONITORING AGENCY NAME(S) AND ADDRESS(ES)}

Headquarters, U.S. Army Corps of Engineers

$441 \mathrm{G}$ Street NW

Washington DC 20314-1000

10. SPONSOR/MONITOR'S ACRONYM(S)

HQUSACE

11. SPONSOR/MONITOR'S REPORT NUMBER(S)

\section{DISTRIBUTION I AVAILABILITY STATEMENT}

Approved for public release. Distribution is unlimited.

\section{SUPPLEMENTARY NOTES}

\section{ABSTRACT}

Current federal, Department of Defense (DoD), and Army energy-efficiency goals require a proactive approach to investment, building operations, and energy savings. Much responsibility for meeting these requirements is assigned to Army installation staff, who often have difficulties identifying and interpreting the applicable mandates. To address this problem, the research group began work to develop an intelligent framework that describes and clarifies interrelationships among energy efficiency, component maintenance and renewal, and mission requirements to support an integrated investment strategy that minimizes total cost of ownership (TCO). The main thrusts of the study were to develop integrated investment decision models, identify DoD facility Energy Use Intensity (EUI) benchmarks in a datascarce environment, and analyze occupant-, system-, and component-level faults contributing to energy inefficiency.

A methodology for developing DoD-specific facility EUIs will serve as a decision framework for actions involving buildings with the highest EUIs. Thus, Army-specific benchmarking results will support more cost-effective component-renewal investment strategies. Altering the timing and grouping of investments can improve the energy efficiency to lower the TCO throughout the facility life cycle. This research will help the Army more effectively implement energy improvements to meet and exceed energy-efficiency requirements.

\section{SUBJECT TERMS}

Energy consumption; Military bases-Energy conservation; Energy conservation-Law and legislation; Electric meters-Power supply; Energy policy; Energy-use modeling

\begin{tabular}{|c|c|c|c|c|c|}
\hline \multicolumn{3}{|c|}{ 16. SECURITY CLASSIFICATION OF: } & \multirow{2}{*}{$\begin{array}{l}\text { 17. LIMITATION } \\
\text { OF ABSTRACT } \\
\text { UU }\end{array}$} & \multirow{2}{*}{$\begin{array}{l}\text { 18. NUMBER } \\
\text { OF PAGES } \\
67\end{array}$} & 19a. NAME OF RESPONSIBLE PERSON \\
\hline $\begin{array}{l}\text { a. REPORT } \\
\text { Unclassified }\end{array}$ & $\begin{array}{l}\text { b. ABSTRACT } \\
\text { Unclassified }\end{array}$ & $\begin{array}{l}\text { c. THIS PAGE } \\
\text { Unclassified }\end{array}$ & & & $\begin{array}{l}\text { 19b. TELEPHONE NUMBER } \\
\text { (include area code) }\end{array}$ \\
\hline
\end{tabular}

